\title{
VIRTUAL MASS AND POLARIZATION
}

\author{
BY \\ M. SCHIFFER AND G. SZEGÖ
}

In this paper we deal with two classical problems of Potential Theory arising in Hydrodynamics and in the Theory of Electricity, respectively.

They are the problems of virtual mass and polarization. In order to understand the dual character of these problems, we shall proceed in a more general way, studying certain formal and other interconnections of the Neumann and Dirichlet problems. The chief definitions, problems, and results are formulated in the somewhat extended introductory Chapter I.

This investigation was carried out under the sponsorship of the Office of Naval Research to which a preliminary report on this subject was submitted (April 17, 1948).

\section{Chapter I. Definitions, problems, Results}

1.1. Virtual mass and polarization. Let us consider an infinite flow of constant density moving in a direction given by the unit vector $h$. By immersing a solid in this fluid, the uniform flow will be disturbed; the disturbance is equivalent with superimposing on the original flow another. If we denote the velocity potential of the superimposed flow by $\phi$, its energy will be given, apart from trivial factors, by the integral

$$
W=\iiint|\operatorname{grad} \phi|^{2} d \tau, \quad d \tau=d x d y d z .
$$

This integral is extended over the exterior of the solid.

Let us consider an infinite electric field whose direction is determined by the unit vector $h$. By placing a conducting solid in this field the uniform field will be disturbed; the disturbance is equivalent with superimposing on the original field another one. If we denote the electric potential of the superimposed field by $\psi$, its energy will be given, apart from trivial factors, by

$$
P=\iiint|\operatorname{grad} \psi|^{2} d \tau \text {. }
$$

This integral is extended again over the whole space exterior to the solid.

Both functions $\phi$ and $\psi$ are harmonic and behave like a dipole at infinity. But the boundary conditions characterizing them are very different. In the first case we have on the surface of the given solid

$$
-\partial \phi / \partial \nu=h \cdot \nu
$$

Presented to the Society April 17, 1948; received by the editors August 10, 1948. 
where $\nu$ is the unit vector in the direction of the exterior normal. In the second case we have

$$
\psi=\boldsymbol{h} \cdot \boldsymbol{r}+\text { const. }
$$

where $\boldsymbol{r}$ is the radius vector. (The additive constant must be chosen properly.) The first problem is a special case of the so-called Neumann problem, the second of the so-called Dirichlet problem.

As to the energies (1) and (2) they are easily verified to be quadratic forms of the components of $h$ :

$$
W=\sum_{i, k=1,2,3} W_{i k} h_{i} h_{k}, \quad P=\sum_{i, k=1,2,3} P_{i k} h_{i} h_{k}
$$

We call $W$ the virtual mass or added mass in the $h$-direction and $P$ the polarization in this direction. The coefficients of these forms depend naturally on the coordinate system used, however, not the invariants of the forms. The simplest among them is the trace

$$
W_{11}+W_{22}+W_{33}=3 W_{m} \text {. }
$$

Recently Professor G. Pólya [14] $\left(^{1}\right)$ has called the invariant $W_{m}$ the average virtual mass and studied the analogous quantity in the case of a two-dimensional flow. In a similar fashion we may introduce the average polarization $P_{m}$ defined by

$$
P_{11}+P_{22}+P_{33}=3 P_{m} \text {. }
$$

The other invariants of the forms (5) may offer also some interest. They are:

$$
\left|\begin{array}{ll}
W_{22} & W_{23} \\
W_{32} & W_{33}
\end{array}\right|+\left|\begin{array}{ll}
W_{33} & W_{31} \\
W_{13} & W_{11}
\end{array}\right|+\left|\begin{array}{ll}
W_{11} & W_{12} \\
W_{21} & W_{22}
\end{array}\right| ; \quad\left|W_{i k}\right|_{i k=1,2,3}
$$

and we have analogous invariants in the case of polarization.

Obviously $W_{m}$ is the mean-value of $W$ on the unit sphere $h_{1}, h_{2}, h_{3}$; a similar remark holds for $P_{m}$.

1.2. Problems. The principal purpose of the present investigation is to find relations of the constants $W_{m}, P_{m}$ depending only on the given solid, to geometrical and other data of the solid. The same question arises concerning the other invariants and even concerning the coefficients of the quadratic forms 1.1 (5). The problem is somewhat similar to that pursued by Professors Pólya and Szegö during the past three years or so concerning the electrostatic capacity and other related quantities $[15,16,18]$. A desirable aim of this investigation might be to establish results for the virtual mass and polarization analogous to certain results obtained for the capacity.

(1) Numbers in brackets refer to the bibliography at the end of the paper. 
We mention in particular two such results. The capacity of an arbitrary solid is never less than the capacity (that is, the radius) of that sphere which has the same volume as the given solid. On the other hand, the capacity of a convex solid is never greater than the radius of that sphere whose so-called Minkowski constant is the same as that of the given solid. The first result was stated by Poincaré [13, pp. 17-22] and proved completely by Faber and Szegö. The second result was found by Szegö in 1930. (Cf. [18].)

In the case of the virtual mass and polarization we are still far from such complete results. But we have found at least inequalities for the quantities in question which become equations in the case of a sphere.

The study of these quantities is greatly facilitated by introducing the expansions of the potentials $\phi$ and $\psi$ at infinity where they behave like a dipole, as observed before. The strength-components of these dipoles in the direction $h$ can be represented in terms of the leading coefficients (those of $r^{-2}$ ) of these potentials; they are certain quadratic forms in $h_{i}$, say,

$$
D=\sum_{i, k=1,2,3} d_{i k} h_{i} h_{k}, \quad E=\sum_{i, k=1,2,3} e_{i k} h_{i} h_{k}
$$

We call them the dipole forms associated with the virtual mass and polarization, respectively. By using Green's formula it is easy to establish the elegant relations the first of which was proved by G. I. Taylor [20]:

$$
W=4 \pi D-V, \quad P=4 \pi E-V
$$

where $V$ is the volume of the solid. The quantity $D$ has a simple meaning; it is proportional to the sum of the virtual mass of the solid and the mass of the displaced fluid. We have found that the quantities $D$ and $E$ show a more regular behavior than the forms $W$ and $P$.

The simplest special case is of course that of a sphere. If the radius is $a$ we have

$$
\begin{array}{lll}
\phi=\frac{a^{3}}{2} \frac{h \cdot \boldsymbol{r}}{r^{3}}, & D=\frac{a^{3}}{2}\left(h_{1}^{2}+h_{2}^{2}+h_{3}^{2}\right)=\frac{a^{3}}{2}, & W=\frac{2 \pi a^{3}}{3}=\frac{V}{2}, \\
\psi=a^{3} \frac{h \cdot \boldsymbol{r}}{r^{3}}, & E=a^{3}\left(h_{1}^{2}+h_{2}^{2}+h_{3}^{2}\right)=a^{3}, & P=\frac{8 \pi a^{3}}{3}=2 V .
\end{array}
$$

Both the virtual mass and polarization have the dimension of a volume.

A list of the most important known cases follows below (1.12-1.14). The list for the virtual mass is rather limited. As to the problem of polarization, it offers much less difficulties. Although we found very few explicit results in this respect, we observed a rather simple relationship of the problem of the polarization to a widely studied problem, namely to that of the influenced electricity $(1.5(14))$. The latter problem has been treated in numerous special cases and from these results the corresponding constants of the polarization can be derived. We prefer however to establish these formulas by direct calcu- 
lation using appropriate curvilinear coordinates, the same which are used customarily in the capacity problem [18]. Such special cases are enumerated in 1.12-1.14 and discussed in Chapter V.

In order to point out the symmetry and the generality of our methods and results we do not restrict ourselves to the study of the special functions $\phi$ and $\psi$. Instead we shall consider the general Neumann and Green functions from a formal point of view. This study is based on the Dirichlet integral and on certain orthogonal systems and kernel functions.

1.3. Notation. Before we start with the discussion of our results, it is advisable to include a few remarks on the notation used in this paper.

In what follows we shall denote the variable point by $p$, its cartesian coordinates by $x_{1}, x_{2}, x_{3}$. In particular cases we may change these symbols into $x, y, z$. The velocity potential $\phi$ associated with the unit vector $h$ will be denoted in particular by $\phi_{1}, \phi_{2}, \phi_{3}$ provided $h$ points in the special directions $x_{1}, x_{2}, x_{3}$, respectively.

We have then for the velocity potential $\phi$ in any direction $h$ :

$$
\phi=h_{1} \phi_{1}+h_{2} \phi_{2}+h_{3} \phi_{3} \text {. }
$$

Forming 1.1 (1) we obtain the first formula in 1.1 (5) using the symbols

(2) $\quad W_{i k}=\iiint \operatorname{grad} \phi_{i} \cdot \operatorname{grad} \phi_{k} d \tau=-\iint \phi_{i} \frac{\partial \phi_{k}}{\partial \nu} d \sigma=-\iint \phi_{k} \frac{\partial \phi_{i}}{\partial \nu} d \sigma$.

Obviously $W_{i k}=W_{k i}$. The expansion of the harmonic function $\phi_{i}$ at infinity has the form

$$
\phi_{i}=r^{-3}\left(d_{i 1} x_{1}+d_{i 2} x_{2}+d_{i 3} x_{3}\right)+\cdots .
$$

Here again $d_{i k}=d_{k i}(2.7$ (11)). We find for the leading term of $\phi$ :

$$
r^{-3} \sum_{i=1}^{3} h_{i}\left(d_{i 1} x_{1}+d_{i 2} x_{2}+d_{i 3} x_{3}\right) .
$$

As we said before the function $\phi$ corresponding to the direction $h$ behaves at $r=\infty$ as a dipole whose strength-component in the direction $h$ is given by the quadratic form $D[1.2(1)]$.

The corresponding quantities for the problem of polarization are denoted by $\psi, \psi_{1}, \psi_{2}, \psi_{3}$ so that

$$
\begin{aligned}
P_{i k}=\iiint \operatorname{grad} \psi_{i} \cdot \operatorname{grad} \psi_{k} d \tau & =-\iint \psi_{i} \frac{\partial \psi_{k}}{\partial \nu} d \sigma \\
& =-\iint \psi_{k} \frac{\partial \psi_{i}}{\partial \nu} d \sigma, \quad P_{i k}=P_{k i}
\end{aligned}
$$

Moreover 


$$
\psi_{i}=r^{-3}\left(e_{i 1} x_{1}+e_{i 2} x_{2}+e_{i 3} x_{3}\right)+\cdots,
$$$$
e_{i k}=e_{k i}
$$

and the electric potential $\psi$ in any direction $h$ will be

$$
\psi=h_{1} \psi_{1}+h_{2} \psi_{2}+h_{3} \psi_{3} .
$$

The strength-component of the dipole which characterizes $\psi$ at $r=\infty$ in the $h$-direction will be represented by the quadratic form $E$ of 1.2 (1).

In some cases we use the vector functions

$$
\phi=i_{1} \phi_{1}+i_{2} \phi_{2}+i_{3} \phi_{3}, \quad \psi=i_{1} \psi_{1}+i_{2} \psi_{2}+i_{3} \psi_{3}
$$

where $i_{1}, i_{2}, i_{3}$ are the unit vectors in the directions of the axes.

Adding to the given velocity potentials those of the uniform flow and field, respectively, we obtain

$$
\Phi=\boldsymbol{h} \cdot \boldsymbol{r}+\boldsymbol{\phi}, \quad \Psi=\boldsymbol{h} \cdot \boldsymbol{r}-\psi
$$

so that the following boundary conditions hold:

$$
\frac{\partial \Phi}{\partial \nu}=0, \quad \Psi=\text { const. }
$$

The special cases of $\Phi$ and $\Psi$ corresponding to the $x_{i}$-direction are denoted by $\Phi_{i}$ and $\Psi_{i}$.

1.4. Classes of harmonic functions and their metric. Let $b$ be a smooth closed surface which bounds the infinite domain $B$. In what follows we deal with four classes (spaces) of functions harmonic in $B$ :

(a) Class $H$ consists of all functions $h(p)$ harmonic in $B$ and having at infinity an expansion of the form

$$
h(p)=a r^{-1}+r^{-3} \sum_{i=1}^{3} a_{i} x_{i}+O\left(r^{-3}\right) .
$$

We call $a$ the strength of source or charge of $h(p)$ at infinity.

(b) Class $H_{0}$ is that part of $H$ for which $a=0$.

(c) Class $\Lambda$ consists of all functions $l(p)$ harmonic in $B$ and having at infinity an expansion of the form

$$
l(p)=\sum_{i=1}^{3} \lambda_{i} x_{i}+a r^{-1}+r^{-3} \sum_{i=1}^{3} a_{i} x_{i}+O\left(r^{-3}\right) .
$$

Obviously $H$ is a part of $\Lambda$.

(d) Class $\Lambda_{0}$ is that part of $\Lambda$ for which $a=0$.

The main instrument of the following study is Dirichlet's integral $D(\lambda, \mu)$ which we defined by the surface integral

$$
D(\lambda, \mu)=-\frac{1}{2} \iint_{b}\left(\lambda \frac{\partial \mu}{\partial \nu}+\mu \frac{\partial \lambda}{\partial \nu}\right) d \sigma .
$$


The normal $\nu$ is directed into the domain $B$. Clearly, if $\lambda \in H, \mu \in H$, this definition is equivalent with the following ones:

$$
\begin{aligned}
D(\lambda, \mu) & =-\iint_{b} \lambda \frac{\partial \mu}{\partial \nu} d \sigma=-\iint_{b} \mu \frac{\partial \lambda}{\partial \nu} d \sigma \\
& =\iiint_{B} \operatorname{grad} \lambda \cdot \operatorname{grad} \mu d \tau, \quad d \tau=d x_{1} d x_{2} d x_{3} .
\end{aligned}
$$

We recognize that (3) has a meaning for the class $\Lambda$ whereas the volume integral in (4) does not. Moreover (3) retains a meaning also in cases when $\lambda$ and $\mu$ have certain singularities in $B$ (outside of $b$ ).

Such singularities arise if we consider "source functions" of the form

$$
h(p)+c r^{-1}, \quad l(p)+c r^{-1}
$$

where $r$ is the distance of the variable point $p$ from a fixed point in $B$ (outside of $b$ ). For such functions (3) still has a meaning.

We interpret $D(\lambda, \mu)$ as the scalar product in a function space, $D(\lambda, \lambda)$ $=D(\lambda)$ as the norm of $\lambda$. This establishes a metric in this space. We observe that the only function $h \in H$ with zero norm is the constant zero.

1.5. Identities and inequalities for Dirichlet integrals. The electric potential $\psi$ of the polarization and the velocity potential $\phi$ of the virtual mass are special functions of class $H_{0}$. In this paper we deal with the following harmonic functions associated with a domain $B$ by special boundary conditions. They are enumerated in dual pairs ( $p$ and $q$ are in $B$ ).

(a) Green's function

$$
G(p, q)=\frac{1}{4 \pi r(p, q)}-g(p, q), \quad G=0 \text { on } b, g \in H(q \text { fixed })
$$

and Neumann's function

(2) $\quad N(p, q)=\frac{1}{4 \pi r(p, q)}+n(p, q), \quad \frac{\partial N}{\partial \nu}=0$ on $b, n \in H(q$ fixed).

(b) The conductor potential $u(p) \in H$ for which $u=1$ on $b$. We call the charge

$$
C=-\frac{1}{4 \pi} \iint_{b} \frac{\partial u}{\partial \nu} d \sigma
$$

the capacity of $b$.

The function $v(p) \in H$ for which $\partial v / \partial \nu=-1$ on $b$. The constant

$$
\Gamma=4 \pi \iint_{b} v d \sigma
$$


related to it is called the stream constant of $b$.

(c) The functions

$$
\Psi_{i}(p)=x_{i}-\psi_{i}(p)=x_{i}-r^{-3} \sum_{k=1}^{3} e_{i k} x_{k}+\cdots, \quad i=1,2,3,
$$

with constant boundary values $\alpha_{i}$, and the functions

$$
\Phi_{i}(p)=x_{i}+\phi_{i}(p)=x_{i}+r^{-3} \sum_{k=1}^{3} d_{i k} x_{k}+\cdots, \quad i=1,2,3,
$$

with vanishing normal derivatives on the boundary [cf. 1.3 (9), (10)]. Obviously $\Psi_{i} \in \Lambda_{0}, \Phi_{i} \in \Lambda_{0}$.

We shall consider various Dirichlet products between these functions. The following typical result is ( $p$ is always the letter of integration)

$$
D\{g(p, q), g(p, s)\}=g(q, s)-I(q, s)
$$

where

$$
\begin{aligned}
I(q, s) & =\frac{1}{16 \pi^{2}} \iint \frac{1}{r(p, s)} \frac{\partial}{\partial \nu}\left[\frac{1}{r(p, q)}\right] d \sigma \\
& =\frac{1}{16 \pi^{2}} \iint \frac{1}{r(p, q)} \frac{\partial}{\partial \nu}\left[\frac{1}{r(p, s)}\right] d \sigma
\end{aligned}
$$

is a "geometric quantity." It is important for the following to make a distinction between potential theoretic quantities which can be determined only by solving a boundary value problem for harmonic functions, and geometric quantities which may be determined by simple integrations of elementary functions over the surface of the domain considered. We shall always consider the latter quantities as elementary and known.

Similarly, we find

$$
D\{n(p, q), n(p, s)\}=n(q, s)-I(q, s)
$$

and

$$
D\{n(p, q), g(p, s)\}=I(q, s) .
$$

Thus the product of the two functions $g$ and $n$ connected with the Neumann and Green functions yields a geometric quantity.

More generally, the product of a function $\lambda$ with prescribed boundary values and a function $\mu$ with prescribed normal derivatives is particularly simple. This is to be expected in view of 1.4 (3).

From (7), (9), and (10) we conclude the interesting inequalities:

$$
\sum g\left(q_{i}, q_{k}\right) t_{i} t_{k} \geqq \sum I\left(q_{i}, q_{k}\right) t_{i} t_{k},
$$




$$
\sum n\left(q_{i}, q_{k}\right) t_{i} t_{k} \geqq \sum I\left(q_{i}, q_{b}\right) t_{i} t_{k} ; \quad i, k=1,2, \cdots, m,
$$

for arbitrarily chosen points $q_{i}$ in $B$ and arbitrary real numbers $t_{i}$. Further, with arbitrary real $t_{i}$ and $t_{i}^{\prime}$,

$$
\begin{aligned}
& 4\left[\sum\left(g\left(q_{i}, q_{k}\right)-I\left(q_{i}, q_{k}\right)\right) t_{i} t_{k}\right]\left[\sum\left(n\left(s_{i}, s_{k}\right)-I\left(s_{i}, s_{k}\right)\right) t_{i}^{\prime} t_{k}^{\prime}\right] \\
& \geqq\left[\sum I\left(q_{i}, s_{k}\right) t_{i} t_{k}^{\prime}\right]^{2} ; \quad i, k=1,2, \cdots, m .
\end{aligned}
$$

If we expand Green's function as a function of $p$ (for fixed $q$ ) into a series about infinity, we obtain

$$
G(p, q)=\frac{1-u(q)}{4 \pi r}+r^{-3} \sum_{i=1}^{3} \frac{1}{4 \pi}\left(\Psi_{i}(q)-\alpha_{i} u(q)\right) x_{i}+O\left(r^{-3}\right)
$$

where $r$ is the distance from a fixed point, say, from the origin, and $\Psi_{i}, \alpha_{i}, u$ have the same meaning as above. Similarly

$$
N(p, q)=\frac{1}{4 \pi r}+\frac{1}{4 \pi r^{3}} \sum_{i=1}^{3} \Phi_{i}(q) x_{i}+O\left(r^{-3}\right) .
$$

This relates the functions $\Psi_{i}$ and $\Phi_{i}$ in which we are interested in the first line to Green's and Neumann's functions and shows that the knowledge of the latter implies already the knowledge of the first pair of functions. In particular the solution for the electrostatic problem of a conductor and a single charge point contains implicitly the solution for the polarization problem.

Finally we note the identities

$$
\begin{aligned}
& D\left(\psi_{i}, \psi_{k}\right)=4 \pi e_{i k}-V \delta_{i k}, \\
& D\left(\phi_{i}, \phi_{k}\right)=4 \pi d_{i k}-V \delta_{i k}, \\
& D\left(\phi_{i}, \psi_{k}\right)=V \delta_{i k}
\end{aligned}
$$

from which the identities 1.2 (2) and the inequality

$$
P W \geqq V^{2}
$$

can be concluded.

Numerous other inequalities are derived from the positive definite character of $D(\lambda)$. For instance

$$
C \Gamma \geqq S^{2}
$$

where $C$ and $\Gamma$ are defined by (3) and (4) and $S$ is the surface area of $b$.

1.6. Orthogonal systems and kernel functions. The study of Dirichlet integrals can be combined with the idea of orthogonal systems and kernel functions.

(a) Let $h(p)$ be a harmonic function of class $H$; then the identities 


$$
D\{G(p, q), h(p)\}=-2^{-1} h(q) ; \quad D\{N(p, q), h(p)\}=2^{-1} h(q)
$$

are valid. Thus if we define the function

$$
k(p, q)=N(p, q)-G(p, q)
$$

we have the identity

$$
D\{k(p, q), h(p)\}=h(q) .
$$

The function $k(p, q)$ is harmonic in $B$ since the poles of Green's and Neumann's functions cancel. It has the remarkable property that its Dirichlet product with every harmonic function reproduces the latter. This shows again that the simultaneous consideration of Dirichlet's and Neumann's problems leads to particularly symmetric and simple formulas. The analogue of $k(p, q)$ was considered in the case of more general partial differential equations of elliptic type by Bergman-Schiffer [3] and called the kernel function.

Let us introduce in the space $H$ an orthogonal system of "coordinates" by the selection of a complete orthonormal set of harmonic functions $\left\{h_{\nu}(p)\right\}$; that is, we require

$$
D\left(h_{v}, h_{\mu}\right)=\delta_{\nu \mu}
$$

and every function $h \in H$ has an expansion

$$
h(p)=\sum_{\nu=1}^{\infty} a_{\nu} h_{\nu}(p), \quad a_{\nu}=D\left(h, h_{\nu}\right) .
$$

It can be shown that this expansion converges in each closed subdomain of $B$. In particular, if we expand the kernel function into a series (5), we find in view of (3)

$$
k(p, q)=\sum_{v=1}^{\infty} h_{\nu}(p) h_{\nu}(q) .
$$

This is a simple representation for the kernel function and permits its construction in terms of a complete orthonormal system.

A particularly useful consequence of (6) is the inequality

$$
k(p, p) \geqq \sum_{\nu=1}^{m}\left[h_{\nu}(p)\right]^{2}
$$

where $m$ is arbitrary. We have here a procedure for obtaining an estimate for $k(p, p)$ which can successively be improved. Now we shall use as the first function of an orthonormal set the conductor potential $(4 \pi C)^{-1 / 2} u(p)$ which is normalized. Then one obtains

$$
k(p, p)=\frac{[u(p)]^{2}}{4 \pi C}+\sum_{v=2}^{\infty}\left[h_{\nu}(p)\right]^{2}
$$


where all $h_{v}(p)$ vanish at infinity at least as $r^{-2}$. Thus, we have

$$
\frac{C}{4 \pi}=\lim _{p \rightarrow \infty}\left\{r^{2} k(p, p)\right\}
$$

and based on this formula and (7) good lower estimates for the capacity may be obtained. (Cf. Pólya-Szegö [16].)

(b) Very similar considerations are possible in the case of the space $\Lambda_{0}$. We form the functions

$$
z_{i}(p)=2^{-1}\left[\Phi_{i}(p)+\Psi_{i}(p)\right], \quad w_{i}(p)=2^{-1}\left[\Phi_{i}(p)-\Psi_{i}(p)\right], \quad i=1,2,3 .
$$

Clearly $z_{i} \in \Lambda_{0}, w_{i} \in H_{0}$ since the singularities at infinity cancel by subtraction. If $h^{0} \in H_{0}$, we have

$$
D\left(z_{i}, h^{0}\right)=0, \quad D\left(w_{i}, h^{0}\right)=2 \pi a_{i}
$$

where $a_{i}$ has the same meaning for $h^{0}$ as in $1.4(1)$.

We may introduce an orthonormal system for the space $H_{0}$ and construct its kernel function $k^{0}(p, q)$. In particular, we might start with the three functions $w_{i}(p)$ and obtain from them by orthonormalization certain functions $\omega_{i}(p)$. If we complete this set by addition of further functions $h_{p}^{0}$ to a complete orthonormal set in $H_{0}$, we know from the second equation (11) that the $h_{v}^{0}$ vanish at infinity at least as $r^{-3}$. Thus, the kernel function

$$
k^{0}(p, q)=\sum_{i=1}^{3} \omega_{i}(p) \omega_{i}(q)+\sum_{\nu=1}^{\infty} h_{\nu}^{0}(p) h_{\nu}^{0}(q)
$$

has near infinity the first right-hand sum as leading term and this permits us to compute $\omega_{i}(p)$ by sending $q \rightarrow \infty$. In a similar fashion the functions $z_{i}(p)$ may be obtained from the kernel function with respect to the whole class $\Lambda_{0}$.

Estimates and approximation procedures for the electric and velocity potentials can be based on these relations. For example, we have the inequality

$$
\pi \sum_{i, k=1,2,3}\left(d_{i k}+e_{i k}\right) \lambda_{i} \lambda_{k} \geqq-D(l)
$$

for every function $l \in \Lambda_{0}$,

The method of orthogonal systems and their kernel functions can immediately be extended to other classes of harmonic functions which have at fixed points prescribed types of singularities. The treatment of the class $\Lambda_{0}$ is important for us because of its connection with the problem of virtual mass and polarization. One recognizes from this treatment the interrelation between the Dirichlet and Neumann problems.

1.7. Inequalities for the virtual mass. Returning to our aim formulated in 1.2 we want to prove inequalities connecting the virtual mass and the 
volume (or other physical or geometrical quantities). This problem fits into the general pattern of the investigation carried out in the paper [16] of PólyaSzegö. Hence it is only natural to attempt to apply similar methods as those employed in that investigation.

In the study of the capacity the main instrument is a systematic use of the principles of Dirichlet and Thomson. They furnish upper and lower bounds for the capacity, respectively. In an analogous fashion upper and lower bounds can be established for the virtual mass. This is possible even for the general Neumann problem as it was pointed out recently by Diaz and Weinstein [4]. In the particular case of the virtual mass we obtain, following the notation of 1.1 ,

$$
W \geqq \frac{\left(\iint f h \cdot \nu d \sigma\right)^{2}}{\iiint|\operatorname{grad} f|^{2} d \tau}
$$

where $f$ is a completely arbitrary function defined in $B$; the surface integral is extended over $b$, the volume integral over $B$.

There is little likelihood for obtaining satisfactory results for the virtual mass in an arbitrary direction $\boldsymbol{h}$ using merely the knowledge of the volume $V$. However Professor Pólya suggested recently [14] the inequality

$$
W_{m} \geqq \frac{V}{2}
$$

for the average virtual mass, an inequality which corresponds to the theorem of Poincaré mentioned in $\$ 1.2$ and means the following: Among all solids of given volume the sphere has the least average virtual mass. We are not able to prove this but we prove various inequalities of a similar kind by using the inequality (1).

How is the function $f$ in (1) chosen? As in the capacity problem we realize first that in certain special cases, in this case for ellipsoids, the velocity potential will have the special form $\phi=\boldsymbol{h} \cdot \boldsymbol{r} g(\alpha)$ provided the $\boldsymbol{h}$-direction is one of the principal directions of the ellipsoid; here $g(\alpha)$ is a function constant on each ellipsoid confocal with the original one which is characterized by $\alpha=\alpha_{0}$. In order to obtain a lower bound for the virtual mass in any $\boldsymbol{h}$-direction we may apply (1) as follows. We choose a certain family of surfaces in $B$ to which the boundary surface $b$ of the solid belongs. (For instance, assuming the star-shaped character of $b$ with respect to an interior point we might choose surfaces similar with respect to this point.) Then we take $f=\boldsymbol{h} \cdot \boldsymbol{r} g(\alpha)$ where $g(\alpha)$ is constant on the surfaces chosen; now we determine the function $g(\alpha)$ so that the right-hand expression in (1) should become a maximum.

We quote two inequalities based on this method. In both cases we assume that $b$ is star-shaped with respect to an interior point $p_{0}$.

(a) Let $r$ be the radius vector issued from $p_{0}$ and leading to the surface $b$, $d \omega$ the surface element of the unit sphere at a point where the normal is 
parallel to the normal of $b$. Then, writing $W_{m}=2 \pi\left(\bar{W}_{m}\right)^{3} / 3$ we have

$$
\bar{W}_{m} \geqq\left(\frac{1}{4 \pi} \iint r^{p} d \omega\right)^{1 / p} \quad \text { for } p \leqq-3 .
$$

The meaning of $\bar{W}_{m}$ is obvious: It is the radius of the sphere whose average virtual mass is the same as that of the given solid. The mean-value on the right-hand side is known to be an increasing function of $p$. For $p=3$ it coincides with the radius $\bar{V}$ of a sphere whose volume is equal to that of the given solid. Hence (3) is still far from the conjectured inequality

$$
\bar{W}_{m} \geqq\left(\frac{1}{4 \pi} \iint r^{3} d \omega\right)^{1 / 3}
$$

Nevertheless (3) will be an equation in the case of a sphere.

(b) A more complicated inequality is the following:

$$
W_{m} \geqq \frac{V}{2}\left(1+\frac{2}{9 V} \iint \nabla\left(r^{3 / 2}\right) d \omega\right)^{-1} ;
$$

here $\nabla(u)$ is Beltrami's operator; it represents the square of the gradient of $u$ on the unit sphere $\left({ }^{2}\right)$.

We may expect for the average polarization $P_{m}$ the inequality

$$
P_{m} \geqq 2 V .
$$

This, as the corresponding inequality for $W_{m}$, can easily be verified for ellipsoids (5.1).

1.8. Another inequality. Another useful inequality, which establishes a relationship between virtual mass $W$ and polarization $P$, has been pointed out in 1.5 (19). It shows that the two quantities $P$ and $W$ cannot be small, compared with $V$, at the same time.

This is an indication for the correctness of the inequalities 1.7 (2), 1.7 (6). From this inequality we conclude a similar inequality for the average polarization and average virtual mass:

$$
P_{m} W_{m} \geqq V^{2} .
$$

Inequality 1.5 (19) becomes an equation in the case of an ellipsoid provided the vector $h$ points in the direction of one of the principal axes.

By means of 1.5 (19) we are able to find lower bounds for the virtual mass in all the cases in which the polarization is known, for instance in the special cases to be dealt with in Chapter V.

As a further illustration of that inequality we mention the special case of two spheres tangent to each other, $h$ being the unit vector in the direction of the central line. In this case (cf. Shiffman-Spencer [17, p. 282])

(2) Cf. W. Blaschke, Vorlesungen über Differentialgeometrie, vol. I, 1921, p. 113. 


$$
W=\left(3 \pi \zeta(3)-\frac{8 \pi}{3}\right) A^{3}
$$

where $A$ is the radius of the spheres, and according to 1.13 (14)

$$
P=\left(16 \pi \zeta(3)-\frac{8 \pi}{3}\right) A^{3}
$$

so that the quantity $\left(^{3}\right)\left(P W / V^{2}\right)^{1 / 6}$ is 1.14 .

1.9. Monotony. We know that the capacity is an increasing set-function. Is the virtual mass or the polarization a function of the same kind? This question must be answered in the negative sense for the virtual mass in general as it was pointed out by G. I. Taylor [20]. We prove however that the answer is affirmative for the dipole forms $D$ and $E$ introduced in 1.2 (1).

A simple consequence of the last result is the following estimations for the virtual mass and polarization:

$$
\begin{gathered}
3 V^{\prime} / 2-V \leqq W \leqq 3 V^{\prime \prime} / 2-V, \\
3 V^{\prime}-V \leqq P \leqq 3 V^{\prime \prime}-V
\end{gathered}
$$

where $V$ is the volume of the solid; $V^{\prime}$ denotes the volume of the largest sphere contained in the given solid, and $V^{\prime \prime}$ the volume of the smallest sphere containing the solid. For the special case of a sphere these inequalities become equations.

1.10. Variational methods. The monotony of the dipole forms $D$ and $E$ is a partial answer to a general problem in potential theory. Given a solid with boundary surface $b$, we may consider various potential theoretic quantities, like Green's function, Neumann's function, capacity, stream constant, polarization, and virtual mass, as functionals of $b$ and ask for their variation with $b$. The method of the Dirichlet integral leads to considerable information in this direction. It permits a unified treatment of the above mentioned quantities. We investigate in Chapter IV the variation of these expressions (except the stream constant) and obtain results of the following type.

(a) Let $b^{\prime}$ be a surface containing $b$. Let $\Sigma$ be the domain between the two surfaces. If $G(p, q)$ denotes Green's function for the exterior of $b$ and $G^{\prime}(p, q)$ for the exterior of $b^{\prime}$, we have the identity:

$$
\begin{aligned}
-G^{\prime}(q, s)= & -G(q, s)+\iiint_{\Sigma} \operatorname{grad} G(p, q) \cdot \operatorname{grad} G(p, s) d \tau \\
& +D^{\prime}\left\{G^{\prime}(p, q)-G(p, q), G^{\prime}(p, s)-G(p, s)\right\} .
\end{aligned}
$$

Here $D^{\prime}$ denotes the Dirichlet integral taken with respect to the exterior of

( $\left.{ }^{3}\right)$ Comparing the quantities $P, W$, and $V$ it seems reasonable to "linearize" the quantity in question, by taking the 6 th root. 
$b^{\prime}$. Exactly the same formula determines the change of Neumann's function $N(p, q)$ of $b$.

(b) Two different types of applications follow from the formula (1). We consider the quadratic forms

$$
Q=\sum_{i, k=1}^{m} g\left(q_{i}, q_{k}\right) t_{i} t_{k}, \quad A=\sum_{i, k=1}^{m} n\left(q_{i}, q_{k}\right) t_{i} t_{k}
$$

and obtain from (1) and the corresponding formula for Neumann's function the result (the meaning of $Q^{\prime}, A^{\prime}$ is obvious):

$$
\begin{aligned}
& Q^{\prime}-Q-\iiint_{\Sigma}\left|\operatorname{grad} \sum_{i=1}^{m} t_{i} G\left(p, q_{i}\right)\right|^{2} d \tau \\
& \quad=D^{\prime}\left\{\sum_{i=1}^{m} t_{i}\left(G^{\prime}\left(p, q_{i}\right)-G\left(p, q_{i}\right)\right)\right\} \geqq 0, \\
& A^{\prime}-A-\iiint_{\Sigma}\left|\operatorname{grad} \sum_{i=1}^{m} t_{i} N\left(p, q_{i}\right)\right|^{2} d \tau \\
& =D^{\prime}\left\{\sum_{i=1}^{m} t_{i}\left(N^{\prime}\left(p, q_{i}\right)-N\left(p, q_{i}\right)\right)\right\} \geqq 0 .
\end{aligned}
$$

Using now certain identities between Dirichlet integrals, we may combine (3) and (4) into the single inequality

$$
\begin{aligned}
{\left[Q^{\prime}-Q-\iiint_{\Sigma}\left|\operatorname{grad} \sum_{i=1}^{m} t_{i} G\left(p, q_{i}\right)\right|^{2} d \tau\right] } \\
\cdot\left[A^{\prime}-A-\iiint_{\Sigma}\left|\operatorname{grad} \sum_{i=1}^{m} t_{i}^{*} N\left(p, q_{i}\right)\right|^{2} d \tau\right] \\
\geqq\left[\iiint_{\Sigma} \operatorname{grad} \sum_{i=1}^{m} t_{i} G\left(p, q_{i}\right) \cdot \operatorname{grad} \sum_{i=1}^{m} t_{i}^{*} N\left(p, q_{i}\right) d \tau\right]^{2}
\end{aligned}
$$

which links the increments of the two different quadratic forms.

A similar reasoning applied to the dipole forms of the polarization and virtual mass leads to the analogous result:

$$
\begin{aligned}
{\left[\sum_{i, k=1}^{3}\left(e_{i k}^{\prime}-e_{i k}\right) h_{i} h_{k}-\frac{1}{4 \pi} \iiint_{\Sigma}\left|\operatorname{grad} \sum_{i=1}^{3} h_{i} \Psi_{i}\right|^{2} d \tau\right] } \\
\cdot\left[\sum_{i, k=1}^{3}\left(d_{i k}^{\prime}-d_{i k}\right) h_{i}^{*} h_{k}^{*}-\frac{1}{4 \pi} \iiint_{\Sigma}\left|\operatorname{grad} \sum_{i=1}^{3} h_{i}^{*} \Phi_{i}\right|^{2} d \tau\right] \\
\geqq\left[\iiint_{\Sigma} \operatorname{grad} \sum_{i=1}^{3} h_{i} \Psi_{i} \cdot \operatorname{grad} \sum_{i=1}^{3} h_{i}^{*} \Phi_{i} d \tau\right]^{2} .
\end{aligned}
$$

It can easily be seen that inequality 1.5 (19) may be obtained from (6) as 
the limiting case if for fixed $b^{\prime}$ the surface $b$ contracts to a point.

Another application of (1) is based on the following remark. If the two surfaces $b$ and $b^{\prime}$ are very near to each other, say in an $\epsilon$-neighborhood, the last Dirichlet integral is of order $\epsilon^{2}$, while the preceding integral is of order $\epsilon$ only. Hence, formulas of the type (1) are useful for a successive approximation procedure for $G^{\prime}$ in terms of $G$. We obtain, in particular, as first order approximation Hadamard's famous variational formula (cf. [5, pp. 303-312]):

$$
\delta G(q, s)=-\iint_{b} \frac{\partial G(p, q)}{\partial \nu} \frac{\partial G(p, s)}{\partial \nu} \delta \nu d \sigma+O\left(\epsilon^{2}\right) .
$$

There is no difficulty in continuing this procedure to higher orders of approximation in $\epsilon$.

In the same way, we obtain the variational formulas for the dipole forms:

$$
\begin{aligned}
& \delta E=\frac{1}{4 \pi} \iint_{b}\left[\frac{\partial}{\partial \nu} \sum_{i=1}^{3} h_{i} \Psi_{i}\right]^{2} \delta \nu d \sigma+O\left(\epsilon^{2}\right), \\
& \delta D=\frac{1}{4 \pi} \iint_{b}\left[\frac{\partial}{\partial \nu} \sum_{i=1}^{3} h_{i} \Phi_{i}\right]^{2} \delta \nu d \sigma+O\left(\epsilon^{2}\right) .
\end{aligned}
$$

Similar variational formulas can be obtained for the potentials $\psi$ and $\phi$ and, if necessary, a higher degree of approximation might also be obtained.

The importance of such variation formulas for the general theory is obvious; they are particularly useful for discovering and checking identities and inequalities. They may serve also to characterize the extremal domain for which a certain quantity of potential theory attains its maximum or minimum.

All variation formulas show a high symmetry and their derivation follows in all cases considered the same pattern of reasoning. It is remarkable that they are all derived from simple formal identities between Dirichlet integrals.

1.11. Nearly spherical domains. Let us consider a variation of the unit sphere. For this case the second variation of the virtual mass has been computed by Szegö [19]. Concerning variational formulas of this kind for other geometrical and physical quantities cf. Pólya-Szegö [16, Chapter VI]. It is instructive to compute the second variation of the polarization and compare the result with the other related results.

We assume that the varied surface has the form

$$
r=(1+f(\theta, \phi)) t=(1+\epsilon \bar{f}(\theta, \phi)) t
$$

where $\boldsymbol{r}=|\boldsymbol{r}| \cdot \boldsymbol{t}$ is the radius vector, $\theta$ and $\phi$ polar coordinates, $\bar{f}(\theta, \phi)$ a fixed function defined on the unit sphere, and $\epsilon \rightarrow 0$.

Let 


$$
f(\theta, \phi)=X_{0}+X_{1}+\cdots+X_{n}+\cdots=\sum_{n=0}^{\infty} X_{n}(\theta, \phi)
$$

be the Laplace series of $f(\theta, \phi)$ where the coefficients of the surface harmonic $X_{n}(\theta, \phi)$ of degree $n$ are of the first order in $\epsilon$. According to [19] we have for the average virtual mass $W_{m}$ of the varied surface $\left(^{4}\right)$ :

$$
\begin{aligned}
\frac{W_{m}}{2 \pi}= & \frac{1}{3}+X_{0}+\sum_{n=0}^{\infty} \frac{1}{4 \pi} \iint X_{n}^{2} d \omega+\sum_{n=2}^{\infty} \frac{3(n-1)(2 n-1)}{2(2 n+1)} \\
& \cdot \frac{1}{4 \pi} \iint X_{n}^{2} d \omega
\end{aligned}
$$

where $d \omega$ is the surface element of the unit sphere. We prove in 4.6

$$
\begin{aligned}
\frac{P_{m}}{8 \pi}= & \frac{1}{3}+X_{0}+\sum_{n=0}^{\infty} \frac{1}{4 \pi} \iint X_{n}^{2} d \omega+\sum_{n=2}^{\infty} \frac{3 n(2 n-1)}{2(2 n+1)} \\
& \cdot \frac{1}{4 \pi} \iint X_{n}^{2} d \omega .
\end{aligned}
$$

It is convenient to compare the radii of the spheres whose average virtual mass, average polarization, capacity, and volume is the same as that of the given solid, respectively. We denote these radii by $\bar{W}_{m}\left[\mathrm{cf} .1 .7\right.$ (a)], $\bar{P}_{m}, C$, and $\bar{V}$. We have then the following remarkable formulas:

(5) $\bar{P}_{m}=1+X_{0}+\sum_{n=1}^{\infty} \frac{1}{4 \pi} \iint X_{n}^{2} d \omega+\sum_{n=2}^{\infty} \frac{3 n(2 n-1)}{2(2 n+1)} \frac{1}{4 \pi} \iint X_{n}^{2} d \omega$,

(6) $\bar{W}_{m}=1+X_{0}+\sum_{n=1}^{\infty} \frac{1}{4 \pi} \iint X_{n}^{2} d \omega+\sum_{n=2}^{\infty} \frac{3(n-1)(2 n-1)}{2(2 n+1)} \frac{1}{4 \pi} \iint X_{n}^{2} d \omega$,

(7) $\quad C=1+X_{0}+\sum_{n=1}^{\infty} \frac{1}{4 \pi} \cdot \iint X_{n}^{2} d \omega+\sum_{n=2}^{\infty} \frac{n-1}{4 \pi} \iint X_{n}^{2} d \omega$,

(8) $\bar{V}=1+X_{0}+\sum_{n=1}^{\infty} \frac{1}{4 \pi} \iint X_{n}^{2} d \omega$.

They suggest the inequalities

$$
(9 C-4 \bar{V}) / 5 \geqq \bar{P}_{m} \geqq \bar{W}_{m} \geqq(9 C+\bar{V}) / 10 \geqq \bar{V}, \quad \bar{P}_{m} \geqq C
$$

and show that the inequality $\bar{W}_{m} \geqq C$ is wrong in general.

1.12. Virtual mass in special cases. Two general methods have been used in order to calculate the virtual mass in special instances. The one is the geometrical method of images, adapted to the hydrodynamical problem( $\left.{ }^{5}\right)$,

(4) In all the subsequent formulas terms of higher than second order in $\epsilon$ have been neglected.

(5) This idea is due to Stokes (cf. Hicks [9, II, p. 50]). 
the other is the use of proper curvilinear coordinates and expansions in terms of corresponding characteristic functions.

(a) Ellipsoid (Green, Stokes, Dirichlet, Clebsch; cf. Lamb [10, pp. 124, $153,155])$. We have in this case

$$
\phi_{i}=2 x_{i} \int_{\rho}^{\infty} \frac{d s}{\left(a_{i}^{2}+s\right) H}, \quad H^{2}=\left(1+\frac{s}{a_{1}^{2}}\right)\left(1+\frac{s}{a_{2}^{2}}\right)\left(1+\frac{s}{a_{3}^{2}}\right)
$$

where $a_{1}, a_{2}, a_{3}$ denote the semiaxes, and

$$
\frac{x_{1}^{2}}{a_{1}^{2}+\rho}+\frac{x_{2}^{2}}{a_{2}^{2}+\rho}+\frac{x_{3}^{2}}{a_{3}^{2}+\rho}=1 .
$$

Introducing the constants

$$
p_{i}=2 \int_{0}^{\infty} \frac{d s}{(a+s) H}
$$

we have

$$
D=\frac{V}{\pi} \sum_{i=1}^{3} \frac{h_{i}^{2}}{4-p_{i}}, \quad W=V \sum_{i=1}^{3} \frac{p_{i}}{4-p_{i}} h_{i}^{2} .
$$

It is easy to prove the following inequality for the average virtual mass

$$
W_{m}=\frac{V}{3} \sum_{i=1}^{3} \frac{p_{i}}{4-p_{i}} \geqq \frac{V}{2} \text {. }
$$

(b) Anchor ring, in the direction of the axis (Hicks [8, p. 648]). Using the notation of Szegö $[18$, p. 344], we have

$$
d_{33}=d_{z}=-\frac{4 a^{3}}{\pi} \sum_{n=0}^{\infty}\left(n^{2}+\gamma\right) x_{n}
$$

where

$$
x_{n}=\frac{Q_{n-1 / 2}^{\prime}\left(\operatorname{ch} \eta_{0}\right)}{P_{n-1 / 2}^{\prime}\left(\operatorname{ch} \eta_{0}\right)} ; \quad \gamma\left(x_{0}-2 \sum_{n=1}^{\infty} \frac{x_{n}}{4 n^{2}-1}\right)=2 \sum_{n=1}^{\infty} \frac{n^{2} x_{n}}{4 n^{2}-1} .
$$

The symbol $\Sigma^{\prime}$ indicates that the term $n=0$ has to be multiplied by $1 / 2$.

From the representations $[18$, p. $344,(2)]$ we conclude that $x_{0}$ is positive and $x_{1}, x_{2}, \cdots$ are negative. Hence $\gamma$ is also negative. Formula (6) is a simplified form of the result of Hicks.

(c) Two spheres, in the direction of the central line (Hicks [7], C. Neumann [12], Herman [6], Basset [2]). We give the result only in the case of two equal spheres; using dipolar coordinates (cf. Szegö $[18$, p. 346]) we have for the spheres $\eta= \pm \beta, \beta>0$, 


$$
d_{11}=d_{x}=a^{3} \sum_{n=1}^{\infty} \frac{(-1)^{n-1}}{(\operatorname{sh} n \beta)^{3}} .
$$

For the flow perpendicular to the direction of the central line, only approximate results have been obtained (cf. Lamb [10, pp. 133-134] and Hicks [7]).

(d) Lens, in the direction of the axis (Shiffman-Spencer [17], special cases by Basset [1] and Herman [6]). The notation being that of 1.13 (e) we have

$$
\begin{aligned}
& d_{33}=d_{z}=\frac{a^{3}}{2} \sin \frac{\pi \alpha}{\alpha+\beta} \\
& \cdot \frac{1}{2 \pi i} \int \csc ^{3} \frac{(\alpha+\beta) t}{\pi} \cdot \csc t\left\{\csc \left(t+\frac{\pi \alpha}{\alpha+\beta}\right)-\csc \left(t-\frac{\pi \alpha}{\alpha+\beta}\right)\right\} d t
\end{aligned}
$$

the line of integration is a vertical line $\operatorname{Re}\{t\}=-\epsilon, 0<(\alpha+\beta) \epsilon<\min (\pi \alpha, \pi \beta)$.

Various interesting special cases have been pointed out by ShiffmanSpencer. We mention two cases which have not been mentioned explicitly by these authors. These are the spherical bowl and two spheres tangent to each other.

The case of the bowl was discussed by Basset [1], not only for the direction of the axis but also in the perpendicular direction. We have in this case

$$
d_{z}=\frac{a^{3}}{2 \pi}\left\{\frac{2}{3}+\frac{\pi-\alpha+2^{-1} \sin 2 \alpha}{\sin ^{3} \alpha}\right\} \text {. }
$$

For $\alpha=\pi$ we obtain the well known case of the disc: $d_{z}=2 a^{3} / 3 \pi$.

In the case of the two tangent spheres, if the radii are $r, r^{\prime}$, we have

$$
d_{z}=\frac{1}{4}\left(\frac{r r^{\prime}}{r+r^{\prime}}\right)^{8}\left\{\lambda^{\prime \prime}\left(\frac{r}{r+r^{\prime}}\right)+\lambda^{\prime \prime}\left(\frac{r^{\prime}}{r+r^{\prime}}\right)-2 \lambda^{\prime \prime}(1)\right\}
$$

where

$$
\lambda(s)=-\frac{\Gamma^{\prime}(s)}{\Gamma(s)}+\frac{\Gamma^{\prime}(1)}{\Gamma(1)}=\sum_{n=0}^{\infty}\left(\frac{1}{n+s}-\frac{1}{n+1}\right) .
$$

In the case $r=r^{\prime}$ mentioned by Shiffman-Spencer,

$$
d_{z}=r^{3} \sum_{n=1}^{\infty} \frac{(-1)^{n-1}}{n^{3}}=\frac{3}{4} r^{3} \zeta(3) \text {. }
$$

1.13. Polarization in special cases. The list of known cases given below is far from being complete. These results can be obtained either from Green's functions (1.5 (14)) or by the use of images or of curvilinear coordinates. We shall use in Chapter $\mathrm{V}$ the latter method in deriving the majority of the formulas. The notation of the curvilinear coordinates and the corresponding characteristic functions is the same used by Szegö [18] except in the case of 
the lens (cf. (e)).

Following the analogous problem of the electrostatic equilibrium we deal with the polarization in these classical cases:

(a) Ellipsoid.

(b) Anchor ring.

(c) Spindle.

(d) Two spheres.

(e) Lens.

Concerning further special cases, cf. Lichtenstein [11, No. 31, pp. 287-291].

(a) Ellipsoid. Using the same notation as in 1.12 (a) we have

$$
\psi_{i}=\frac{2 x_{i}}{p_{i}} \int_{\rho}^{\infty} \frac{d s}{\left(a_{i}^{2}+s\right) H}
$$

so that

$$
E=\frac{V}{\pi} \sum_{i=1}^{3} \frac{h_{i}^{2}}{p_{i}}, \quad P=V \sum_{i=1}^{3} \frac{4-p_{i}}{p_{i}} h_{i}^{2}
$$

It is interesting to compare these formulas with the corresponding formulas for the virtual mass. It is easy to verify the following inequality for the average polarization:

$$
P_{m}=\frac{V}{3} \sum_{i=1}^{3} \frac{4-p_{i}}{p_{i}} \geqq 2 V .
$$

Inequality 1.5 (19) can also be verified.

(b) Anchor ring. Using peripolar coordinates $\eta, \theta, \phi$ we have for the torus $\eta=\eta_{0}\left(\eta_{0}>0\right)$ in the direction of the axis (z-direction) the following electric potential:

(4) $\quad \psi_{3}=\psi_{z}=\frac{4 a}{\pi}(2 \operatorname{ch} \eta-2 \cos \theta)^{1 / 2} \sum_{n=1}^{\infty} n \frac{Q_{n-1 / 2}\left(\operatorname{ch} \eta_{0}\right)}{P_{n-1 / 2}\left(\operatorname{ch} \eta_{0}\right)} P_{n-1 / 2}(\operatorname{ch} \eta) \sin n \theta$, and for the polarization in the same direction:

$$
e_{33}=e_{z}=\frac{16 a^{3}}{\pi} \sum_{n=1}^{\infty} n^{2} \frac{Q_{n-1 / 2}\left(\operatorname{ch} \eta_{0}\right)}{P_{n-1 / 2}\left(\operatorname{ch} \eta_{0}\right)} .
$$

In the direction of the $x$-axis we have

$$
\psi_{1}=\psi_{x}=-\frac{4 a}{\pi}(2 \operatorname{ch} \eta-2 \cos \theta)^{1 / 2} \operatorname{sh} \eta \cos \phi
$$

$$
\cdot \sum_{n=0}^{\infty} \frac{Q_{n-1 / 2}^{\prime}\left(\operatorname{ch} \eta_{0}\right)}{P_{n-1 / 2}^{\prime}\left(\operatorname{ch} \eta_{0}\right)} P_{n-1 / 2}^{\prime}(\operatorname{ch} \eta) \cos n \theta
$$


and

$$
\begin{aligned}
e_{11}=e_{22}=e_{x}=e_{y} & =-\frac{8 a^{3}}{\pi} \sum_{n=0}^{\infty}\left(n^{2}-\frac{1}{4}\right) \frac{Q_{n-1 / 2}^{\prime}\left(\operatorname{ch} \eta_{0}\right)}{P_{n-1 / 2}^{\prime}\left(\operatorname{ch} \eta_{0}\right)} \\
& =-\frac{8 a^{3}}{\pi} \sum_{n=0}^{\infty}\left(n^{2}-\frac{1}{4}\right) X_{n}
\end{aligned}
$$

where $X_{n}$ has the same meaning as in 1.12 (b); the symbol $\Sigma^{\prime}$ used also in the same sense as there. Compare $e_{x}=e_{y}$ with $2 d_{z}$ where $d_{z}$ is defined by $1.12(6)$.

It is also interesting to compare the previous formulas with the expressions of the conductor potential and of the electrostatic capacity (Szegö [18, p. $344])$ :

$$
\begin{aligned}
& u=\frac{2}{\pi}(2 \operatorname{ch} \eta-2 \cos \theta)^{1 / 2} \sum_{n=0}^{\infty} \frac{Q_{n-1 / 2}\left(\operatorname{ch} \eta_{0}\right)}{P_{n-1 / 2}\left(\operatorname{ch} \eta_{0}\right)} P_{n-1 / 2}(\operatorname{ch} \eta) \cos n \theta, \\
& C=\frac{4 a}{\pi} \sum_{n=0}^{\infty} \frac{Q_{n-1 / 2}\left(\operatorname{ch} \eta_{0}\right)}{P_{n-1 / 2}\left(\operatorname{ch} \eta_{0}\right)} .
\end{aligned}
$$

(c) Spindle. If we use dipolar coordinates $\eta, \theta, \phi$ the spindle is characterized by the condition $\theta=\theta_{0}, 0<\theta_{0}<\pi$. In the direction of the axis which is the $x$-direction, the following representation of the electric potential and of the polarization holds:

$$
\begin{aligned}
\psi_{x} & =a(2 \operatorname{ch} \eta-2 \cos \theta)^{1 / 2} \int_{-\infty}^{\infty} \frac{L_{q}\left(\cos \theta_{0}\right) L_{q}(-\cos \theta)}{L_{q}\left(-\cos \theta_{0}\right)} \cdot \frac{q \sin q \eta}{\operatorname{ch} q \pi} d q \\
e_{x} & =4 a^{3} \int_{-\infty}^{\infty} \frac{L_{q}\left(\cos \theta_{0}\right)}{L_{q}\left(-\cos \theta_{0}\right)} \frac{q^{2}}{\operatorname{ch} q \pi} d q .
\end{aligned}
$$

In the direction of the $y$-axis we have

$$
\psi_{y}=a(2 \operatorname{ch} \eta-2 \cos \theta)^{1 / 2} \sin \theta \cos \phi
$$

$$
\cdot \int_{-\infty}^{\infty} \frac{L_{q}^{\prime}\left(\cos \theta_{0}\right) L_{q}^{\prime}(-\cos \theta)}{L_{q}^{\prime}\left(-\cos \theta_{0}\right)} \frac{\cos q \eta}{\operatorname{ch} q \pi} d q
$$

$$
e_{y}=e_{z}=2 a^{3} \int_{-\infty}^{\infty} \frac{L_{q}^{\prime}\left(\cos \theta_{0}\right)}{L_{q}^{\prime}\left(-\cos \theta_{0}\right)} \frac{q^{2}+1 / 4}{\operatorname{ch} q \pi} d q .
$$

For the sake of comparison we quote the formulas for the conductor potential and the electrostatic capacity (Szegö $[18$, p. 345]):

$$
\begin{aligned}
& u=\frac{1}{2}(2 \operatorname{ch} \eta-2 \cos \theta)^{1 / 2} \int_{-\infty}^{\infty} \frac{L_{q}\left(\cos \theta_{0}\right) L_{q}(-\cos \theta)}{L_{q}\left(-\cos \theta_{0}\right)} \frac{\cos q \eta}{\operatorname{ch} q \pi} d q, \\
& C=a \int_{-\infty}^{\infty} \frac{L_{q}\left(\cos \theta_{0}\right)}{L_{q}\left(-\cos \theta_{0}\right)} \frac{d q}{\operatorname{ch} q \pi} .
\end{aligned}
$$


(d) Two spheres. Using dipolar coordinates the two spheres are characterized by the conditions $\eta=\alpha<0$ and $\eta=\beta>0$. In the direction of the central line ( $x$-direction) we have for the electric potential

$$
\begin{aligned}
\psi_{x}=(2 \operatorname{ch} \eta-2 \cos \theta)^{1 / 2} & \sum_{n=0}^{\infty}\left\{4 a N \frac{\operatorname{ch} N \alpha \cdot e^{N(\eta-\beta)}-\operatorname{ch} N \beta \cdot e^{N(\alpha-\eta)}}{e^{N \delta}-e^{-N \delta}}\right. \\
& \left.-2 p \frac{\operatorname{sh} N \alpha \cdot e^{N(\eta-\beta)}-\operatorname{sh} N \beta \cdot e^{N(\alpha-\eta)}}{e^{N \delta}-e^{-N \delta}}\right\} P_{n}(\cos \theta)
\end{aligned}
$$

and for the polarization

$$
\begin{aligned}
e_{x}= & 16 a^{3} \sum_{n=0}^{\infty} N^{2} \frac{\operatorname{ch~} N \alpha \cdot e^{-N \beta}+\operatorname{ch} N \beta \cdot e^{N \alpha}}{e^{N \delta}-e^{-N \delta}} \\
& -8 a^{2} p \sum_{n=0}^{\infty} N \frac{\operatorname{sh} N \alpha \cdot e^{-N \beta}+\operatorname{sh} N \beta \cdot e^{N \alpha}}{e^{N \delta}-e^{-N \delta}}
\end{aligned}
$$

where the constant $p$ has to be determined by the condition that the sum in (14) vanishes for $\eta=\theta=0$. Here $N=n+1 / 2, \delta=\beta-\alpha$.

By a simple transformation we obtain

$$
\begin{gathered}
e_{x}=a^{3} \sum_{m=0}^{\infty}[f(m \delta+\beta)+f(m \delta-\alpha)+2 f(m \delta+\delta)] \\
-\frac{a^{3}\left[\sum_{m=0}^{\infty}(g(m \delta+\beta)-g(m \delta-\alpha))\right]^{2}}{\sum_{m=0}^{\infty}[h(m \delta+\beta)+h(m \delta-\alpha)-2 h(m \delta+\delta)]} ; \\
f(x)=\frac{1+\operatorname{ch}^{2} x}{\operatorname{sh}^{3} x}=h^{\prime \prime}(x), \quad g(x)=\frac{\operatorname{ch} x}{\operatorname{sh}^{2} x}=-h^{\prime}(x), \quad h(x)=\frac{1}{\operatorname{sh} x} .
\end{gathered}
$$

In the $y$-direction we have for the electric potential

$$
\begin{gathered}
\psi_{y}=(2 \operatorname{ch} \eta-2 \cos \theta)^{1 / 2} \cdot 4 a \sum_{n=0}^{\infty} \frac{\operatorname{sh} N \beta \cdot e^{N(\alpha-\eta)}-\operatorname{sh} N \alpha \cdot e^{N(\eta-\beta)}}{e^{N \delta}-e^{-N \delta}} \\
\cdot \sin \theta P_{n}^{\prime}(\cos \theta) \cos \phi,
\end{gathered}
$$

and for the polarization in the $y$ - and $z$-directions:

$$
\begin{aligned}
e_{y} & =e_{z}=4 a^{3} \sum_{n=0}^{\infty}\left(N^{2}-\frac{1}{4}\right) \frac{\operatorname{ch} N(\alpha+\beta)-e^{-N \delta}}{\operatorname{sh} N \delta} \\
& =a^{3} \sum_{m=0}^{\infty}\left[\operatorname{sh}^{-3}(m \delta+\beta)+\operatorname{sh}^{-3}(m \delta-\alpha)-2 \operatorname{sh}^{-3}(m \delta+\delta)\right] .
\end{aligned}
$$


In case of equal spheres we have $\alpha=-\beta$, and

$$
e_{x}=2 a^{3} \sum_{n=1}^{\infty} f(n \beta), \quad e_{y}=e_{z}=2 a^{3} \sum_{n=1}^{\infty} \frac{(-1)^{n-1}}{(\operatorname{sh} n \beta)^{3}} .
$$

It is interesting to note that (cf. $1.12(8)$ ):

$$
2 d_{x}=e_{y}=e_{z} .
$$

(e) Lens. In this case it seems advisable to change the notation used in Szegö $[18$, p. 347$]$. The following table compares the symbols used by various authors:

\begin{tabular}{|l|c|c|}
\hline Szegö [18] & $\theta_{1}$ & $\theta_{2}$ \\
\hline Shiffman-Spencer [17] & $\alpha-\beta$ & $2 \pi-\beta$ \\
\hline present paper & $\alpha$ & $2 \pi-\beta$ \\
\hline
\end{tabular}

We assume that $\alpha$ and $\beta$ are positive, $\alpha<\pi, \alpha+\beta \leqq 2 \pi$. The "upper" surface $\theta=\alpha$ of the lens is in the upper half-space. The "lower" surface $\theta=2 \pi-\beta$ is in the upper or lower half-space. The domain outside of the lens is characterized by the condition

$$
-\beta<\theta<\alpha .
$$

The exterior angle is $\alpha+\beta$. The volume is

$$
V=\frac{a^{3} \pi}{6}\left\{(2-\cos \alpha) \cot \frac{\alpha}{2} \csc ^{2} \frac{\alpha}{2}+(2-\cos \beta) \cot \frac{\beta}{2} \csc ^{2} \frac{\beta}{2}\right\} \text {. }
$$

We employ the function (Szegö $[18$, p. 347])

$$
\begin{aligned}
f_{q}(\eta) & =\frac{\pi}{\operatorname{ch} q \pi} L_{q}(-\operatorname{ch} \eta)=\frac{\pi}{\operatorname{ch} q \pi} F\left(\frac{1}{2}+i q, \frac{1}{2}-i q ; 1 ;-\operatorname{sh}^{2} \frac{\eta}{2}\right) \\
& =\int_{-\infty}^{\infty}\left(2 \operatorname{ch} \eta^{\prime}+2 \operatorname{ch} \eta\right)^{-1 / 2} \cos q \eta^{\prime} d \eta^{\prime} ; q \text { real, }|\operatorname{Im} \eta|<\pi
\end{aligned}
$$

so that the functions

$$
\begin{aligned}
& (2 \operatorname{ch} \eta-2 \cos \theta)^{1 / 2} f_{q}(\eta) e^{ \pm q \theta} \\
& (2 \operatorname{ch} \eta-2 \cos \theta)^{1 / 2} f_{q}^{\prime}(\eta) e^{ \pm q \theta+i \phi}
\end{aligned}
$$

are harmonic.

We give here the result for the general lens and in the next section for its numerous special cases. 
In the $z$-direction which is the axis of the lens we have for the electric potential:

$$
\psi_{z}=(2 \operatorname{ch} \eta-2 \cos \theta)^{1 / 2}
$$

$$
\begin{array}{r}
\cdot \frac{a}{\pi} \int_{-\infty}^{\infty} f_{q}(\eta) \\
-\frac{q[\operatorname{sh} q(\alpha-\theta) \operatorname{sh} q(\beta-\pi)-\operatorname{sh} q(\alpha-\pi) \operatorname{sh} q(\beta+\theta)]}{\operatorname{sh} q(\alpha+\beta)} \\
\left.-\frac{p[\operatorname{sh} q(\alpha-\theta) \operatorname{ch} q(\beta-\pi)+\operatorname{ch} q(\alpha-\beta) \operatorname{sh} q(\beta+\theta)]}{\operatorname{sh} q(\alpha+\beta)}\right) d q
\end{array}
$$

and for the dipole coefficients:

$$
\begin{array}{r}
\boldsymbol{e}_{z}=4 a^{3} \int_{-\infty}^{\infty}\left(\frac{q^{2}[\operatorname{sh} q(\pi-\alpha) \operatorname{ch} q \beta+\operatorname{ch} q \alpha \operatorname{sh} q(\pi-\beta)]}{\operatorname{sh} q(\alpha+\beta) \operatorname{ch} q \pi}\right. \\
\left.-\frac{p q[\operatorname{ch} q(\alpha-\pi) \operatorname{ch} q \beta-\operatorname{ch} q(\beta-\pi) \operatorname{ch} q \alpha]}{\operatorname{sh} q(\alpha+\beta) \operatorname{ch} q \pi}\right) d q
\end{array}
$$

Here the constant $p$ has to be determined by the condition that the integral in (24) vanishes for $\eta=\theta=0$.

By a simple transformation we obtain

$$
\begin{gathered}
e_{z}=4 a^{3} \int_{-\infty}^{\infty} q^{2} \frac{\operatorname{sh} q \pi \operatorname{ch} q(\beta-\alpha)+\operatorname{sh} q(\pi-\alpha-\beta)}{\operatorname{sh} q(\alpha+\beta) \operatorname{ch} q \pi} d q \\
-\frac{4 a^{3}\left[\int_{-\infty}^{\infty} q \operatorname{th} q \pi \frac{\operatorname{sh} q(\alpha-\beta)}{\operatorname{sh} q(\alpha+\beta)} d q\right]^{2}}{\int_{-\infty}^{\infty} \frac{\operatorname{sh} q \pi \operatorname{ch} q(\beta-\alpha)+\operatorname{sh} q(\alpha+\beta-\pi)}{\operatorname{sh} q(\alpha+\beta) \operatorname{ch} q \pi} d q} .
\end{gathered}
$$

In the $x$-direction we have for the electric potential

$$
\begin{aligned}
\psi_{x}= & (2 \operatorname{ch} \eta-2 \cos \theta)^{1 / 2} \\
& \cdot \frac{a}{\pi} \int_{-\infty}^{\infty} f_{q}^{\prime}(\eta) \frac{\operatorname{sh} q(\theta-\alpha) \operatorname{ch} q(\pi-\beta)-\operatorname{ch} q(\pi-\alpha) \operatorname{sh} q(\theta+\beta)}{\operatorname{sh} q(\alpha+\beta)} \\
& \cdot d q \cos \phi
\end{aligned}
$$

and for the dipole coefficient:

$$
\begin{aligned}
e_{x}=e_{y} & =2 a^{3} \int_{-\infty}^{\infty}\left(q^{2}+\frac{1}{4}\right) \frac{\operatorname{sh} q \alpha \operatorname{ch} q(\pi-\beta)+\operatorname{ch} q(\pi-\alpha) \operatorname{sh} q \beta}{\operatorname{sh} q(\alpha+\beta) \operatorname{ch} q \pi} d q \\
& =2 a^{3} \int_{-\infty}^{\infty}\left(q^{2}+\frac{1}{4}\right) \frac{\operatorname{sh} q \pi \operatorname{ch} q(\alpha-\beta)+\operatorname{sh} q(\alpha+\beta-\pi)}{\operatorname{sh} q(\alpha+\beta) \operatorname{ch} q \pi} d q
\end{aligned}
$$

For the sake of comparison we note the expression for the electrostatic capacity (Szegö [18, p. 348]): 


$$
C=a \int_{-\infty}^{\infty} \frac{\operatorname{sh} q \pi \operatorname{ch} q(\alpha-\beta)+\operatorname{sh} q(\alpha+\beta-\pi)}{\operatorname{sh} q(\alpha+\beta) \operatorname{ch} q \pi} d q .
$$

1.14. Special cases of the lens. (a) Spherical bowl, $\alpha+\beta=2 \pi, 0<\alpha<\pi$ (cf. Basset [1, p. 294, p. 296]):

$$
e_{z}=a^{3}\left\{f^{\prime \prime}(\alpha)-f^{\prime \prime}(\pi)-\left[f^{\prime}(\alpha)\right]^{2} / f(\alpha)\right\}
$$

where

$$
f(\alpha)=\int_{-\infty}^{\infty}\left[\frac{\operatorname{ch} q(\pi-\alpha)}{\operatorname{ch} q \pi}\right]^{2} d q=\frac{1}{\pi}+\left(1-\frac{\alpha}{\pi}\right) \frac{1}{\sin \alpha} .
$$

Furthermore

$$
\begin{aligned}
e_{x} & =e_{y}=2 a^{3} \int_{-\infty}^{\infty}\left(q^{2}+\frac{1}{4}\right)\left[\frac{\operatorname{ch} q(\pi-\alpha)}{\operatorname{ch} q \pi}\right]^{2} d q \\
& =\frac{a^{3}}{2}\left\{f^{\prime \prime}(\alpha)+f^{\prime \prime}(\pi)+f(\alpha)\right\} .
\end{aligned}
$$

We have $f^{\prime \prime}(\pi)=(3 \pi)^{-1}$. The capacity is $C=a f(\alpha)$. We observe that (cf. 1.12 (10)) $2 d_{z}=e_{x}=e_{y}$.

(b) Circular disc, limiting case of (a), $\alpha \rightarrow \pi$ :

$$
e_{z}=0, \quad e_{x}=e_{y}=\frac{4 a^{3}}{3 \pi} .
$$

(c) Half spherical (thin) shell, special case of (a), $\alpha=\pi / 2$ :

$$
e_{z}=a^{3}\left(\frac{1}{2}-\frac{4}{3 \pi}+\frac{1}{2+\pi}\right), \quad e_{x}=e_{y}=a^{3}\left(\frac{1}{2}+\frac{2}{3 \pi}\right) .
$$

(d) Symmetrical lens, $\alpha=\beta$; in this case

(6) $\quad \psi_{z}=(2 \operatorname{ch} \eta-2 \cos \theta)^{1 / 2} \frac{a}{\pi} \int_{-\infty}^{\infty} f_{q}(\eta) \frac{q \operatorname{sh} q(\pi-\alpha)}{\operatorname{sh} q \alpha} \operatorname{sh} q \theta d q$,

$$
e_{z}=4 a^{3} \int_{-\infty}^{\infty} q^{2}[\operatorname{th} q \pi \operatorname{cth} q \alpha-1] d q
$$

$$
\psi_{x}=-(2 \operatorname{ch} \eta-2 \cos \theta)^{1 / 2} \frac{a}{\pi} \int_{-\infty}^{\infty} f_{q}^{\prime}(\eta) \frac{\operatorname{ch} q(\pi-\alpha)}{\operatorname{ch} q \alpha} \operatorname{ch} q \theta d q \cdot \cos \phi
$$

(9)

$$
e_{x}=e_{y}=2 a^{3} \int_{-\infty}^{\infty}\left(q^{2}+\frac{1}{4}\right)(1-\operatorname{th} q \pi \text { th } q \alpha) d q
$$

For the sake of comparison we mention that 


$$
C=a \int_{-\infty}^{\infty}(1-\operatorname{th} q \pi \text { th } q \alpha) d q .
$$

(e) Two spheres of radii $r$ and $r^{\prime}$ tangent to each other, $\alpha \rightarrow 0, \beta \rightarrow 0, a \rightarrow 0$, $a / \alpha \rightarrow r, a / \beta \rightarrow r^{\prime}:$

$$
\begin{aligned}
e_{z}=\left(\frac{r r^{\prime}}{r+r^{\prime}}\right)^{3}\left\{\lambda^{\prime \prime}\left(\frac{r}{r+r^{\prime}}\right)+\lambda^{\prime \prime}\left(\frac{r^{\prime}}{r+r^{\prime}}\right)+2 \lambda^{\prime \prime}(1)\right. & \\
& \left.-\frac{\left[\lambda^{\prime}\left(r /\left(r+r^{\prime}\right)\right)-\lambda^{\prime}\left(r^{\prime} /\left(r+r^{\prime}\right)\right)\right]^{2}}{\lambda\left(r /\left(r+r^{\prime}\right)\right)+\lambda\left(r^{\prime} /\left(r+r^{\prime}\right)\right)}\right\}
\end{aligned}
$$

where $\lambda(s)$ has the same meaning as in $1.12(12)$; moreover

$$
e_{x}=e_{y}=\frac{1}{2}\left(\frac{r r^{\prime}}{r+r^{\prime}}\right)^{3}\left\{\lambda^{\prime \prime}\left(\frac{r}{r+r^{\prime}}\right)+\lambda^{\prime \prime}\left(\frac{r^{\prime}}{r+r^{\prime}}\right)-2 \lambda^{\prime \prime}(1)\right\} .
$$

Again (cf. $1.12(11)): 2 d_{z}=e_{x}=e_{y}$. Finally, we compare these results with the expression for the capacity (Szegö $[18$, p. 346, (4)]):

$$
C=\frac{r r^{\prime}}{r+r^{\prime}}\left\{\lambda\left(\frac{r}{r+r^{\prime}}\right)+\lambda\left(\frac{r^{\prime}}{r+r^{\prime}}\right)\right\} \text {. }
$$

For two equal spheres, $r=r^{\prime}$,

$$
e_{z}=2 r^{3} \lambda^{\prime \prime}(1)=4 r^{3} \zeta(3), \quad e_{x}=e_{y}=(3 / 2) r^{3} \zeta(3) .
$$

Chapter II. IDentities AND INEQUALITIES For DiRIChlet INTEgRals

In this chapter the formal transformations and identities are worked out which are of importance for the rest of the paper. A somewhat more general approach is chosen which considers also Green's and Neumann's functions and their properties. One reason for this is the fact that the polarization problem may be reduced to the problem of determining Green's function and the virtual mass problem to that of Neumann's function as will be shown. Another reason is the greater symmetry of treatment which is achieved by this broader approach.

2.1. Green's and Neumann's functions. Using the notation of Chapter I we introduce the two fundamental functions of potential theory associated with a given surface $b$ and study their Dirichlet products with functions $h \in H$.

Green's function

$$
G(p, q)=\frac{1}{4 \pi r(p, q)}-g(p, q)
$$

is harmonic in $B$ except for $p=q$ and vanishes if either argument point comes upon the boundary $b ; g \in H$ as a function of $p$ ( $q$ fixed). Green's function is 
symmetric in $p$ and $q$.

Neumann's function

$$
N(p, q)=\frac{1}{4 \pi r(p, q)}+n(p, q)
$$

is harmonic in $B$ except for $p=q$ and has in each argument a vanishing normal derivative on $b ; n \in H$. Neumann's function, too, is symmetric in $p$ and $q$.

It follows from the extremum principle on harmonic functions that $G(p, q)>0$ if $p, q$ are in $B$. It is less obvious that also

$$
N(p, q) \geqq 0 \quad p, q \text { in } B .
$$

To prove this, let $q$ be fixed and let us suppose there were a sub-domain $B^{\prime} \subset B$ in which $N(p, q)<0$. The boundary of $B^{\prime}$ is made up of either surfaces where $N=0$ or of parts of $b$. Thus, we have along the whole boundary $b^{\prime}$ of $B^{\prime}$ the equation

$$
N(p, q) \frac{\partial}{\partial \nu} N(p, q)=0, \quad \quad p \in b^{\prime}, q \text { fixed. }
$$

Thus, by Green's identity, we would have

$$
\iiint_{B^{\prime}}|\operatorname{grad} N(p, q)|^{2} d \tau_{p}=0 .
$$

This is a contradiction, since Neumann's function is not identically zero. Thus, (3) is proved. From the extremum principle, we infer also

$$
N(p, q) \geqq G(p, q)
$$

for the difference between these two fundamental functions:

$$
k(p, q)=N(p, q)-G(p, q)
$$

is regular in $B$ and non-negative on $b$.

Let now $h(p)$ be an arbitrary function of class $H$. Using the representation 1.4 (3) for the Dirichlet integral, we conclude the identities:

$$
D\{G(p, q), h(p)\}=-h(p) / 2
$$

and

$$
D\{N(p, q), h(p)\}=h(q) / 2 .
$$

From (8) and (9) it follows that the non-negative function $k(p, q)$ defined by (7) has the elegant property:

$$
D\{k(p, q), h(p)\}=h(q) .
$$

Thus, $k(p, q)$ is a reproducing kernel for the class $H$ with respect to Dirichlet 
multiplication. It is very remarkable that the combination (7) of Green's and Neumann's functions, which is of the class $H$ itself, behaves in many respects simpler than each of its components.

2.2. The kernel function. The importance of the function $k(p, q)$ may best be recognized by the following consideration. Let $\left\{h_{\nu}(p)\right\}$ be a complete orthonormal set of functions with respect to the class $H$ and the Dirichlet metric; that is, we assume

$$
D\left(h_{\nu}, h_{\mu}\right)=\delta_{\nu \mu}= \begin{cases}1 & \text { for } \nu=\mu \\ 0 & \text { for } \quad \nu \neq \mu\end{cases}
$$

and if $h$ is an arbitrary function of $H$, we have

$$
h(p)=\sum_{\nu=1}^{\infty} a_{\nu} h_{\nu}(p), \quad a_{\nu}=D\left(h, h_{\nu}\right)
$$

where the sum converges uniformly in each closed sub-domain of $B$. Expanding, in particular, $k(p, q)$ for fixed $q$, into such a series in $h_{\nu}(p)$ we find in view of $2.1(10)$

$$
k(p, q)=\sum_{\nu=1}^{\infty} h_{\nu}(p) h_{\nu}(q)
$$

This shows that $k(p, q)$ is the kernel function of every complete orthonormal system in $H$. From this fact, a simple construction of this particular combination of Green's and Neumann's functions can be derived.

If we specialize in $2.1(10): h(p)=k(p, s)$ with $s \in B$, we obtain

$$
D\{k(p, q), k(p, s)\}=k(q, s) .
$$

Applying now Schwarz's inequality to 2.1 (10) and using the identity (4), we find

$$
[h(q)]^{2} \leqq k(q, q) D(h) .
$$

Equality holds only for $h(p)=k(p, q)$ and this property might serve to define the kernel function by an extremum problem. The great importance of the kernel function for the class $H$ is clearly illustrated by (5).

We insert in (5) the function $h(q)=k(q, s)$ and have, in view of (4),

$$
[k(q, s)]^{2} \leqq k(q, q) \cdot k(s, s)
$$

which permits an estimate of the function $k(q, s)$, depending on two points, in terms of the function $k(q, q)$ which depends only on the single point $q$. The same inequality can be obtained directly from (3) by means of Cauchy's inequality.

Inequality (6) may easily be generalized by the following consideration. We choose $m$ points $q_{i} \in B$ and consider the function 


$$
h(p)=\sum_{i=1}^{m} \dot{t}_{i} k\left(p, q_{i}\right)
$$

which is evidently of class $H$. Since its norm is non-negative, we have in view of (4)

$$
D(h)=\sum_{i, k=1}^{n} k\left(q_{i}, q_{k}\right) t_{i} t_{k} \geqq 0,
$$

that is, the matrix $\left(\left(k\left(q_{i}, q_{k}\right)\right)\right)$ is semi-definite. This result clearly implies (6). Inequalities of the type (8) are characteristic for the study of harmonic functions by means of the Dirichlet integral and we shall encounter them frequently in the following sections.

2.3. Identities for $g(p, q)$ and $n(p, q)$. In this section we form various Dirichlet products involving the regular parts $g(p, q)$ and $n(p, q)$ of Green's and Neumann's function. This leads to further inequalities of the type $2.2(8)$. find

(a) Let $q$ and $s$ be two arbitrary points in $B$. Using Green's identity, we

$$
\begin{aligned}
D\{g(p, q), g(p, s)\} & =-\iint_{b} g(p, q) \frac{\partial}{\partial \nu} g(p, s) d \sigma \\
& =-\frac{1}{4 \pi} \iint_{b} \frac{1}{r(p, q)} \frac{\partial}{\partial \nu} g(p, s) d \sigma .
\end{aligned}
$$

By means of the identity

$$
\frac{1}{4 \pi} \iint_{b}\left[h(p) \frac{\partial}{\partial \nu}\left(\frac{1}{r(p, q)}\right)-\frac{1}{r(p, q)} \frac{\partial}{\partial \nu} h(p)\right] d \sigma=h(q)
$$

which is valid for every function $h(p)$ of class $H$ we arrive at

$$
\begin{aligned}
D\{g(p, q), g(p, s)\} & =g(q, s)-\frac{1}{4 \pi} \iint_{b} g(p, s) \frac{\partial}{\partial \nu}\left(\frac{1}{r(p, q)}\right) d \sigma \\
& =g(q, s)-\frac{1}{16 \pi^{2}} \iint_{b} \frac{1}{r(p, s)} \frac{\partial}{\partial \nu}\left(\frac{1}{r(p, q)}\right) d \sigma .
\end{aligned}
$$

Let us denote by $\widetilde{B}$ the finite complement of $B$ which is enclosed by $b$; the normal $\nu$ is relative to $\widetilde{B}$ the exterior one. We may, therefore, write

$$
\begin{aligned}
I(q, s) & =\frac{1}{16 \pi^{2}} \iint_{b} \frac{1}{r(p, s)} \frac{\partial}{\partial \nu}\left(\frac{1}{r(p, q)}\right) d \sigma \\
& =\frac{1}{16 \pi^{2}} \iiint_{\widetilde{B}} \operatorname{grad} \frac{1}{r(p, q)} \cdot \operatorname{grad} \frac{1}{r(p, s)} d \tau
\end{aligned}
$$

and this quantity is symmetric in $q$ and $s$; it depends only on the geometry 
of the surface $b$. We obtain finally

$$
D\{g(p, q), g(p, s)\}=g(q, s)-I(q, s) .
$$

Consider now the following function of the class $H$; let $q_{i}$ be $m$ points in $B$ and let

We have in view of $(5)$ :

$$
h(p)=\sum_{i=1}^{m} t_{i} g\left(p, q_{i}\right)
$$

$$
D(h)=\sum_{i, k=1}^{m}\left[g\left(q_{i}, q_{k}\right)-I\left(q_{i}, q_{k}\right)\right] t_{i} t_{k} \geqq 0 .
$$

Obviously

$$
\sum_{i, k=1}^{m} I\left(q_{i}, q_{k}\right) t_{i} t_{k}=\iiint_{\widetilde{B}}\left|\operatorname{grad} \sum_{i=1}^{m} \frac{t_{i}}{r\left(p, q_{i}\right)}\right|^{2} d \tau \geqq 0 .
$$

Thus, we proved that the matrix $\left(\left(g\left(q_{i}, q_{k}\right)\right)\right)$ is positive-definite and gave for it a lower bound in terms of the matrix $\left(\left(I\left(q_{i}, q_{k}\right)\right)\right)$. In particular, we obtained the following inequality:

$$
(g(p, q))^{2} \leqq g(p, p) \cdot g(q, q) .
$$
find

(b) Performing similar transformations with Neumann's function, we

$$
D\{n(p, q), n(p, s)\}=n(q, s)-I(q, s)
$$

which is completely analogous to (5). We are led to the corresponding inequality

$$
\sum_{i, k=1}^{m}\left[n\left(q_{i}, q_{k}\right)-I\left(q_{i}, q_{k}\right)\right] t_{i} t_{k} \geqq 0
$$

and, in particular, to the inequality

$$
(n(p, q))^{2} \leqq n(p, p) \cdot n(q, q) .
$$

- Since, by definition,

$$
k(p, q)=n(p, q)+g(p, q)
$$

we may derive from (7) and (11) the inequality

$$
\sum_{i, k=1}^{m} k\left(q_{i}, q_{k}\right) t_{i} t_{k} \geqq 2 \sum_{i, k=1}^{m} I\left(q_{i}, q_{k}\right) t_{i} t_{k}
$$

which is much stronger than 2.2 (8).

Finally, we form the scalar product 


$$
\begin{aligned}
D\{n(p, q), g(p, s)\} & =-\iint_{b} g(p, s) \frac{\partial}{\partial \nu} n(p, q) d \sigma \\
& =\frac{1}{16 \pi^{2}} \iint_{b} \frac{1}{r(p, s)} \frac{\partial}{\partial \nu} \frac{1}{r(p, q)} d \sigma
\end{aligned}
$$

that is, by virtue of (4)

$$
D\{n(p, q), g(p, s)\}=I(q, s) .
$$

This result is particularly simple since the right-hand side contains a geometric quantity which can be computed directly without solving boundary value problems for harmonic functions. Cf. the remark to 1.5 (10).

(c) As an application of these identities let us consider the following harmonic function $h \in H$ :

$$
\begin{aligned}
h(p)=\sum_{i=1}^{m} t_{i} g\left(p, q_{i}\right)+\tau \sum_{i=1}^{m^{\prime}} t_{i}^{\prime} n\left(p, q_{i}^{\prime}\right), & \\
& q_{i} \in B, q_{i}^{\prime} \in B ; t_{i}, t_{i}^{\prime}, \tau \text { real. }
\end{aligned}
$$

Its norm is non-negative whatever values $t_{i}, t_{i}{ }^{\prime}$, and $\tau$ are chosen; hence, because of (5), (10), and (15), we obtain the discriminant condition

$$
\begin{aligned}
4\left[\sum_{i, k=1}^{m}\left(g\left(q_{i}, q_{k}\right)-I\left(q_{i}, q_{k}\right)\right) t_{i} t_{k}\right]\left[\sum_{i, k=1}^{m^{\prime}}\left(n\left(q_{i}^{\prime}, q_{k}^{\prime}\right)-I\left(q_{i}^{\prime}, q_{k}^{\prime}\right)\right) t_{i}^{\prime} t_{k}^{\prime}\right] \\
\geqq\left[\sum_{i=1}^{m} \sum_{k=1}^{m^{\prime}} I\left(q_{i}, q_{k}^{\prime}\right) t_{i} t_{k}^{\prime}\right]^{2} .
\end{aligned}
$$

This is a remarkable interrelation between the two definite quadratic forms (7) and (11). We see that the smallness of the one form implies a large value of the other, provided the purely geometric quantity on the right-hand side of (17) is given. We see again that the simultaneous study of Green's and Neumann's functions leads to elegant results, as exemplified by the kernel function, the identity (15), and the inequality (17).

2.4. The capacity of the surface $b$. We define the conductor potential of the domain $B$ as the harmonic function $u(p) \in H$ which has on $b$ the boundary values 1 . It may be interpreted as the potential of an electrostatic equilibrium charge distribution over the conducting surface $b$; the charge $C$ at infinity is the capacity of the conductor.

Let $h(p) \in H$, then one finds easily

$$
D(u, h)=4 \pi a
$$

where $a$ is the charge of $h$, and in particular,

$$
D(u)=4 \pi C .
$$


From (1) we conclude: The class of all functions $h \in H$ which are orthogonal to the conductor potential is identical with the class of all functions $h \in H$ with vanishing charge $a$.

We may use this result in order to obtain an interesting relation between the conductor potential and the kernel function $k(p, q)$. Since $h_{1}(p)$ $=(4 \pi C)^{-1 / 2} u(p)$ is normalized with respect to the Dirichlet metric, it can be chosen as the first element of a complete orthonormal system $\left\{h_{\nu}\right\}$ for $B$. By the preceding theorem, all $h_{\nu}(p)$ with $\nu>1$ will be then without charge, that is, they vanish at infinity at least as $r^{-2}$. Using the representation 2.2 (3) for the kernel function, we find

$$
k(p, q)=\frac{u(p) u(q)}{4 \pi C}+\sum_{\nu=2}^{\infty} h_{\nu}(p) h_{\nu}(q) .
$$

In view of the asymptotic behavior of the $h_{\nu}(p)$ at infinity, we find

$$
\lim _{p \rightarrow \infty}\{r k(p, q)\}=\frac{u(q)}{4 \pi} .
$$

This is a representation of the conductor potential in terms of the kernel function.

We obtain from (3) the particular instance

$$
k(p, p)=\frac{(u(p))^{2}}{4 \pi C}+\sum_{\nu=2}^{\infty}\left(h_{\nu}(p)\right)^{2} .
$$

Let $\left\{f_{\nu}\right\}$ be now an arbitrary complete orthonormal set in $B$; we have also

$$
\sum_{\nu=1}^{\infty}\left(f_{\nu}(p)\right)^{2}=\frac{(u(p))^{2}}{4 \pi C}+\sum_{\nu=2}^{\infty}\left(h_{\nu}(p)\right)^{2} .
$$

Let $b_{\nu}$ be the charge of $f_{\nu}(p)$ at infinity; comparing the terms of order $r^{-2}$ on both sides of this identity, we find

$$
\sum_{v=1}^{\infty} b_{v}^{2}=\frac{C}{4 \pi}
$$

If a finite number of orthonormal functions $f_{\nu}, \nu=1,2, \cdots, n$, is known, obviously

$$
\sum_{\nu=1}^{m} b_{\nu}^{2} \leqq \frac{C}{4 \pi}
$$

In the case $m=1$, we have: For every $h \in H$ with the charge a

$$
4 \pi a^{2}(D(h))^{-1} \leqq C
$$

and equality holds only if $h$ is the conductor potential $u$. 
This result is nothing but the well known principle of Thomson that among all potentials with given charge $a$ at infinity the conductor potential has the minimum energy $a^{2} C^{-1} / 2$. (Cf. $[16,2.1]$.) It may be derived in various ways and is frequently used to obtain from an arbitrarily chosen $h \in H$ a lower bound for the capacity $C$ of $b$. By means of (7) we are able to improve this estimate systematically. Having chosen a function $f_{1} \in H$ arbitrarily and made our first estimate, we introduce a second function $f_{2}$ which is orthogonal to $f_{1}$ and obtain because of (8) a better estimate for $C$. If necessary, this procedure may be continued indefinitely. Thus, the well known inequality (9) appears in our formulation as the first step in a continuously improving approximation procedure. In the subsequent sections other potential theoretic quantities will be represented in a similar way by means of appropriate kernel functions. The significance of such a representation will be clear then from the above remarks.

Finally, we consider the Dirichlet product

$$
D\{g(p, q), u(p)\}=-\frac{1}{4 \pi} \iint_{b} \frac{1}{r(p, q)} \frac{\partial}{\partial \nu} u(p) d \sigma=u(q) .
$$

If we expand $g(p, q)$ at infinity into a series

$$
g(p, q)=\frac{a(q)}{r}+\cdots
$$

we find because of (1)

$$
4 \pi a(q)=D\{g(p, q), u(p)\}=u(q) .
$$

Thus, we may obtain $u(q)$ by expanding $g(p, q)$ into a series (1.5 (14)):

$$
\lim _{p \rightarrow \infty}\{r g(p, q)\}=\frac{u(q)}{4 \pi} .
$$

Another formula expressing the conductor potential by means of Green's function is the following:

$$
u(q)=\iint_{b} \frac{\partial}{\partial \nu} G(p, q) d \sigma_{p} .
$$

2.5. The stream constant of $b$. It is of some interest to consider the dual concept to conductor potential and capacity. We define the function $v(p)$ $\in H$ which has on $b$ the constant normal derivative -1 . Its source strength at infinity is

$$
a=-\frac{1}{4 \pi} \iint_{b} \frac{\partial v}{\partial \nu} d \sigma=\frac{S}{4 \pi}
$$

where $S$ is the surface area of $b$. If $h \in H$ we have 


$$
D(h, v)=-\iint_{b} h \frac{\partial v}{\partial \nu} d \sigma=\iint_{b} h d \sigma .
$$

In particular,

$$
D(v)=\iint_{b} v d \sigma=\frac{\Gamma}{4 \pi}
$$

where $\Gamma$ is a characteristic constant of the surface $b$ and will be called the stream constant of $b$. It is the dual concept of the capacity. By Green's formula, we have

$$
v(q)=\iint_{b} N(p, q) d \sigma_{p}
$$

which shows by $2.1(3)$ that $v(q)$ is non-negative. From (3) and (4) we conclude

$$
\Gamma=4 \pi \iint_{p \in b} \iint_{q} \in_{b} N(p, q) d \sigma_{q} d \sigma_{p} \geqq 0 .
$$

Let now $h \in H$ be arbitrary but with a prescribed value $\iint_{b} h d \sigma=s$. It is easy to estimate $D(h)$ by means of $\Gamma$. This shows the important role of this concept in the general theory of harmonic functions. In fact, applying Schwarz's inequality in (2) and using (3), we find

$$
4 \pi\left(\iint_{b} h d \sigma\right)^{2} \leqq \Gamma D(h) .
$$

Thus, we obtain the following formula which is dual to 2.4 (9):

$$
4 \pi s^{2}(D(h))^{-1} \leqq \Gamma .
$$

As in the case of the capacity this formula may be refined with the aid of the kernel function. In fact, let $h_{1}(p)=(\Gamma / 4 \pi)^{-1 / 2} v(p)$ be the first element of a complete orthonormal system $\left\{h_{\nu}\right\}$ in $B$. We have, by definition 2.2 (3):

$$
k(p, q)=\frac{4 \pi v(p) v(q)}{\Gamma}+\sum_{v=2}^{\infty} h_{\nu}(p) h_{\nu}(q) .
$$

In view of (2) all $h_{\nu}$ for $\nu \geqq 2$ have vanishing surface integrals so that

$$
4 \pi \iint_{p \in b} \iint_{q \in b} k(p, q) d \sigma_{q} d \sigma_{p}=\Gamma .
$$

This follows also easily from 2.1 (7) and from (5). Let now $\left\{f_{\nu}\right\}$ be an arbitrary complete orthonormal system and let

$$
\iint_{b} f_{\nu} d \sigma=s_{\nu}
$$


We have from (9)

$$
\Gamma=4 \pi \sum_{\nu=1}^{\infty} s_{v}^{2}
$$

and this identity permits a successive approximation of the stream constant.

The Dirichlet product of the dual expressions $u$ and $v$ is again particularly simple:

$$
D(u, v)=\iint_{b} d \sigma=S .
$$

Thus it has a simple geometric meaning. Using Schwarz's inequality, 2.4 (2) and (3), we obtain the elegant inequality $1.5(20)$.

2.6. The class $\Lambda$. (a) In order to deal with the virtual mass and polarization we need an extension of the class $H$ which we called in 1.4 the class $\Lambda$. This class arises from the class $H$ by adjunction of the three special functions $x_{i}$ of class $\Lambda$. We find by the definition 1.4 (3):

$$
D\left(x_{i}, x_{k}\right)=-\frac{1}{2} \iint_{b}\left(x_{i} \cos \left(\nu, x_{k}\right)+x_{k} \cos \left(\nu, x_{i}\right)\right) d \sigma=-V \delta_{i k}
$$

where $V$ is the volume of the solid $\widetilde{B}$ enclosed by $b$. Thus the three functions $x_{i} \in \Lambda$ have a negative norm so that there exists an infinity of functions $l(p) \in \Lambda$ with non-positive norm.

This makes clear that it is impossible to determine a complete orthonormal system in the usual sense for $\Lambda$. We may, however, determine a system $\left\{l_{\nu}\right\}$ with the property

$$
D\left(l_{\nu}, l_{\mu}\right)= \pm \delta_{\nu \mu}
$$

and such that each function $l \in \Lambda$ may be represented in the form

$$
l=\sum_{\nu=1}^{\infty} a_{\nu} l_{\nu}, \quad a_{\nu}= \pm D\left(l, l_{\nu}\right),
$$

where the sign in the expression for $a_{\nu}$ must be the same as that in (2) for $\nu=\mu$. We shall call $\left\{l_{\nu}\right\}$ a complete orthogonal system in $\Lambda$.

(b) If we want to determine the scalar products of all functions $l \in \Lambda$ with Green's and Neumann's functions, it is sufficient in view of 2.1 (8) and 2.1 (9) to compute the corresponding products with the $x_{i}$ only. We find by definition 1.4 (3)

$$
D\left\{G(p, q), x_{i}\right\}=-\frac{1}{2} \iint_{b} x_{i} \frac{\partial}{\partial \nu} G(p, q) d \sigma=-\frac{1}{2} \psi_{i}^{0}(q), \quad i=1,2,3 .
$$

Here $\psi_{i}^{0}(q) \in H$ and has on $b$ the boundary values $x_{i}$. If we subtract from $\psi_{i}^{0}$ an appropriate multiple of the conductor potential we can cancel its 
charge at infinity and obtain a new function $\psi_{i}(q) \in H_{0}$. It is identical with the electric potential of the polarization problem. This function $\psi_{i}(q)=\psi_{i}^{0}(q)$ $-\alpha_{i} u(q)$ has on $b$ the boundary values $x_{i}-\alpha_{i}$ where the $\alpha_{i}$ are certain characteristic constants.

The geometric significance of the $\alpha_{i}$ is best concluded from the identity (2.4 (1) and (2))

$$
0=D\left(u, \psi_{i}\right)=D\left(u, \psi_{i}^{0}\right)-\alpha_{i} D(u)=-\iint_{b} \psi_{i}^{0} \frac{\partial u}{\partial \nu} d \sigma+\alpha_{i} \iint_{b} u \frac{\partial u}{\partial \nu} d \sigma
$$

so that using the boundary values of $\psi_{i}^{0}$ and $u$, we obtain

$$
\alpha_{i}=\left(\iint_{b} \frac{\partial u}{\partial \nu} d \sigma\right)^{-1} \iint_{b} x_{i} \frac{\partial u}{\partial \nu} d \sigma .
$$

Since $-(1 / 4 \pi)(\partial u / \partial \nu)$ is the density of the equilibrium charge on the conducting surface $b$, we see that the point with the coordinates $\alpha_{i}$ is the centroid of this equilibrium charge distribution on $b$.

(c) We have further

$$
D\left\{N(p, q), x_{i}\right\}=-\frac{1}{2} \iint_{b} N(p, q) \cos \left(\nu, x_{i}\right) d \sigma=-\frac{1}{2} \phi_{i}(q) .
$$

Here $\phi_{i}(q) \in H$ and has on $b$ the normal derivative $-\cos \left(\nu, x_{i}\right)$. From this fact, we conclude

$$
\iint_{b} \frac{\partial}{\partial \nu} \phi_{i} d \sigma=-\iint_{b} \cos \left(\nu, x_{i}\right) d \sigma=0,
$$

that is, $\phi_{i}(q) \in H_{0}$ and is identical with the velocity potential of the virtual mass problem.

We define as in $1.3(9)$ :

$$
\Psi_{i}=x_{i}-\psi_{i}, \quad \Phi_{i}=x_{i}+\phi_{i}
$$

They are of class $\Lambda_{0}$ and $\Psi_{i}$ has constant boundary values $\alpha_{i}$ on $b$ while $\Phi_{i}$ has a vanishing normal derivative there.

2.7. Identities for the $\Psi_{i}$ and $\Phi_{i}$. The functions $\Psi_{i}$ and $\Phi_{i}$ have a very simple behavior on the boundary $b$ of $B$ and permit, therefore, an easy computation of their Dirichlet products with other harmonic functions.

(a) Let $h \in H$; using the notation 1.4 (1) we have

$$
\begin{aligned}
D\left(\Psi_{i}, h\right) & =-\frac{1}{2} \iint_{b}\left(h \frac{\partial}{\partial \nu} \Psi_{i}+\Psi_{i} \frac{\partial}{\partial \nu} h\right) d \sigma \\
& =4 \pi a \alpha_{i}-\frac{1}{2} \iint_{b}\left(h \frac{\partial}{\partial \nu} \Psi_{i}-\Psi_{i} \frac{\partial}{\partial \nu} h\right) d \sigma .
\end{aligned}
$$


Thus, we used the particular boundary values of $\Psi_{i}$ in order to transform the Dirichlet integral into the standard form of Green's identity. Now we have if $h \in H, \lambda \in H$,

$$
\iint_{b}\left(h \frac{\partial \lambda}{\partial \nu}-\lambda \frac{\partial h}{\partial \nu}\right) d \sigma=0 .
$$

Also we find (notation as in $1.4(1)$ )

$$
\frac{1}{2} \iint_{b}\left(h \frac{\partial x_{i}}{\partial \nu}-x_{i} \frac{\partial h}{\partial \nu}\right) d \sigma=2 \pi a_{i},
$$

extending the integration instead of over $b$, over a very large sphere. Thus, we have finally

$$
D\left(\Psi_{i}, h\right)=4 \pi a \alpha_{i}-2 \pi a_{i} .
$$

In the same fashion, we obtain

$$
D\left(\Phi_{i}, h\right)=2 \pi a_{i} .
$$

(b) In order to determine the Dirichlet products of $\Psi_{i}$ and $\Phi_{i}$ with all elements of $\Lambda$ it is sufficient to compute only their products with $x_{k}$. Using the same methods as before, we find

$$
D\left(\Psi_{i}, x_{k}\right)=-\frac{1}{2} \iint_{b}\left(x_{k} \frac{\partial \Psi_{i}}{\partial \nu}-\Psi_{i} \frac{\partial x_{k}}{\partial \nu}\right) d \sigma=-2 \pi e_{i k}
$$

and

$$
D\left(\Phi_{i}, x_{k}\right)=-\frac{1}{2} \iint_{b}\left(\Phi_{i} \frac{\partial x_{k}}{\partial \nu}-x_{k} \frac{\partial \Phi_{i}}{\partial \nu}\right) d \sigma=-2 \pi d_{i k} .
$$

It is further obvious from the definition of the Dirichlet integral that

$$
D\left(\Psi_{i}, \Psi_{k}\right)=D\left(\Phi_{i}, \Phi_{k}\right)=0 .
$$

We may transform these two equations by means of (4), (6), (5), and (7) into

$$
\begin{aligned}
& D\left(x_{i}, \Psi_{k}\right)-D\left(\psi_{i}, \Psi_{k}\right)=-2 \pi e_{k i}+2 \pi e_{i k}=0, \\
& D\left(x_{i}, \Phi_{k}\right)+D\left(\phi_{i}, \Phi_{k}\right)=-2 \pi d_{k i}+2 \pi d_{i k}=0 ;
\end{aligned}
$$

this proves the symmetry of the matrices $\left(\left(e_{i k}\right)\right)$ and $\left(\left(d_{i k}\right)\right)$ :

$$
e_{i k}=e_{k i}, \quad d_{i k}=d_{k i} \text {. }
$$

As a special instance of (4), we have the identity

$$
D\left(\Psi_{i}, \phi_{k}\right)=-2 \pi d_{i k}
$$

and adding this to (6), we find in view of 2.6 (9) 


$$
D\left(\Psi_{i}, \Phi_{k}\right)=-2 \pi\left(e_{i k}+d_{i k}\right) .
$$

Thus, we determined all Dirichlet products between the $\Psi_{i-}$ and $\Phi_{k}$-functions.

From these identities, we arrive easily at the multiplication table for the functions $\psi_{i}$ and $\phi_{k}$ given in $1.5(16)-(18)$. We have to use 2.6 (1), (6), (7), (8), and (13); moreover we obtain the identities 1.2 (2).

(c) Finally we apply (4) to the conductor potential $u(p)$ and find

$$
D\left(\Psi_{i}, u\right)=4 \pi C \alpha_{i}-2 \pi \gamma_{i}
$$

where $\gamma_{i}$ denotes the special values of $a_{i}$ if $h=u$. On the other hand, we find directly, in view of the sourceless character of $\Psi_{i}$ :

$$
D\left(\Psi_{i}, u\right)=-\frac{1}{2} \iint_{b}\left(\alpha_{i} \frac{\partial u}{\partial \nu}+\frac{\partial \Psi_{i}}{\partial \nu}\right) d \sigma=2 \pi C \alpha_{i} .
$$

Hence, we have the simple relation between the $\alpha_{i}$ and $\gamma_{i}$ :

$$
\gamma_{i}=C \alpha_{i}
$$

and may write (14) in the alternate form

$$
D\left(\Psi_{i}, u\right)=2 \pi \gamma_{i}
$$

From (5), we obtain also

$$
D\left(\Phi_{i}, u\right)=2 \pi \gamma_{i}
$$

2.8. Orthogonality relations and the kernel function. In this section the identities of 2.7 will be applied in order to construct the functions $\Psi_{i}$ and $\Phi_{i}$ by means of orthogonal systems. The concept of the kernel function will play a central role.

We start forming the following two linear combinations of $\Psi_{i}$ and $\Phi_{i}$ :

$$
\begin{aligned}
& Z_{i}(p)=\left[\Phi_{i}(p)+\Psi_{i}(p)\right] / 2=x_{i}+\left(\phi_{i}-\psi_{i}\right) / 2, \\
& w_{i}(p)=\left[\Phi_{i}(p)-\Psi_{i}(p)\right] / 2=\left(\phi_{i}+\psi_{i}\right) / 2 .
\end{aligned}
$$

Using the notation 1.4 (1) for an arbitrary $h \in H$, we obtain, in view of 2.7 (4) and $2.7(5)$,

$$
\begin{aligned}
& D\left(Z_{i}, h\right)=2 \pi a \alpha_{i}, \\
& D\left(w_{i}, h\right)=-2 \pi a \alpha_{i}+2 \pi a_{i} .
\end{aligned}
$$

Since $w_{k} \in H_{0}$ we infer from (3) and (4):

$$
D\left(Z_{i}, w_{k}\right)=0, \quad D\left(w_{i}, w_{k}\right)=\pi\left(d_{i k}+e_{i k}\right) .
$$

For the sake of completeness, we point out the following consequence of 2.7 (8) and 2.7 (13):

$$
D\left(z_{i}, z_{k}\right)=-\pi\left(d_{i k}+e_{i k}\right)
$$


In view of (3) and (4) we have the theorems:

I. The functions $z_{i}, i=1,2,3$, are orthogonal to all functions of $H_{0}$.

II. All functions in $H_{0}$ which are orthogonal to $w_{i}, i=1,2,3$, vanish at infinity at least as $r^{-3}$.

Consider now the matrix

$$
M=\pi\left(\left(d_{i k}+e_{i k}\right)\right)
$$

which is symmetric and, in view of $1.2(2)$, positive-definite. Let $O=\left(\left(o_{i k}\right)\right)$ be the matrix which satisfies the equation

$$
O M O^{\prime}=\left(\left(\delta_{i k}\right)\right)
$$

Then, introducing the functions

$$
\xi_{i}(p)=\sum_{k=1}^{3} o_{i k} z_{k}(p), \quad \omega_{i}(p)=\sum_{k=1}^{3} o_{i k} w_{k}(p)
$$

we recognize easily from (5), (6), and (8) that they satisfy the relations

$$
D\left(\xi_{i}, \xi_{k}\right)=-\delta_{i k}, \quad D\left(\omega_{i}, \omega_{k}\right)=\delta_{i k} .
$$

Let now $\omega_{1}, \omega_{2}, \omega_{3}$ be the three first elements of a complete orthonormal system $\left\{h_{\nu}^{0}\right\}$ in $H_{0}$. If $k_{0}(p, q)$ is the corresponding kernel function, we have

$$
k_{0}(p, q)=\sum_{i=1}^{3} \omega_{i}(p) \omega_{i}(q)+\sum_{\nu=4}^{\infty} h_{\nu}^{0}(p) h_{\nu}^{0}(q) .
$$

According to Theorem II all functions $h_{\nu}^{0}(p)$ for $\nu>3$ vanish at infinity at least as $r^{-3}$. Thus, the first right-hand sum is the leading term at infinity. This permits us to compute the $\omega_{i}(p)$ and, therefore, the $w_{i}(p)$ by means of the kernel function. In particular, we have

$$
\begin{aligned}
k_{0}(p, p) & =\sum_{i=1}^{3}\left(\omega_{i}(p)\right)^{2}+\sum_{v=4}^{\infty}\left(h_{\nu}^{0}(p)\right)^{2} \\
& =\frac{1}{4 \pi} \sum_{i, k=1,2,3}\left(d_{i k}+e_{i k}\right) x_{i} x_{k} r^{-6}+O\left(r^{-6}\right)
\end{aligned}
$$

which yields a systematic approximation for the quadratic form

$$
Q=\frac{1}{4 \pi} \sum_{i, k=1,2,3}\left(d_{i k}+e_{i k}\right) x_{i} x_{k}
$$

In the same manner we may connect the $\xi_{i}(p)$ with a kernel function with respect to the class $\Lambda_{0}$. Let, in fact, $\left\{f_{\nu}^{0}\right\}$ be a complete orthonormal system in $H_{0}$. By adjunction of the three functions $\xi_{i}(p)$ we obtain a complete orthogonal system for $\Lambda_{0}$ as is easily seen from the Theorem I and (10). Thus, the kernel function of this system has the form 


$$
K_{0}(p, q)=-\sum_{i=1}^{3} \xi_{i}(p) \xi_{i}(q)+\sum_{\nu=1}^{\infty} f_{\nu}^{0}(p) f_{\nu}^{0}(q)
$$

and the characteristic property

$$
D\left\{K_{0}(p, q), l^{0}(p)\right\}=l^{0}(q) \quad \text { for every } l^{0} \in \Lambda_{0} .
$$

The leading term of $K_{0}(p, q)$ at infinity is the first right-hand sum. One might now construct the kernel function in the following way: take, for example, the three functions $x_{i}$ and the system $\left\{f_{v}^{0}\right\}$ and orthogonalize them. From such a construction one obtains a representation for the $\xi_{i}(p)$ in terms of the elements of an orthogonal system.

We find easily from the definitions (8) and (9)

$$
K_{0}(p, p)=-\sum_{i, k=1,2,3} \mu_{i k} x_{i} x_{k}+\text { finite terms at infinity, }
$$

with $M^{-1}=\left(\left(\mu_{i k}\right)\right)$. We have further

$$
\lim _{x_{l} \rightarrow \infty}\left\{x_{l}^{-1} K_{0}(p, q)\right\}=-\sum_{i=1}^{3} o_{i l} \xi_{i}(q)=-\sum_{i=1}^{3} \mu_{l i} Z_{i}(q)
$$

if the two other $x$-coordinates of $p$ remain finite. From formulas (16) and (17) approximations for $M$ and the $Z_{i}(p)$ may be obtained.

2.9. Identities for $\psi_{i}, \phi_{i}$, Green's and Neumann's functions. In this section we deal with the Dirichlet products between the functions $\psi_{i}, \phi_{i}$, on the one hand, and Green's and Neumann's functions, on the other. We find, in this way, an important result which shows that the functions $\Psi_{i}$ and $\Phi_{i}$ may be obtained directly from Green's and Neumann's functions as coefficients of a certain expansion. Also we derive from our identities certain inequalities by applying Schwarz's inequality in the usual way.

We find by easy transformations

$$
D\left\{\psi_{i}(p), g(p, q)\right\}=\psi_{i}(q)-J_{i}(q)
$$

where $J_{i}(q)$ is a purely geometric quantity:

$$
\begin{aligned}
J_{i}(q) & =\frac{1}{4 \pi} \iint_{b} x_{i} \frac{\partial}{\partial \nu}\left(\frac{1}{r(p, q)}\right) d \sigma=\frac{1}{4 \pi} \iint_{b} \frac{1}{r(p, q)} \frac{\partial x_{i}}{\partial \nu} d \sigma \\
& =\frac{1}{4 \pi} \iiint_{\widetilde{B}} \frac{\partial}{\partial x_{i}}\left(\frac{1}{r(p, q)}\right) d \tau .
\end{aligned}
$$

In the same way:

$$
\begin{aligned}
D\left\{\psi_{i}(p), n(p, q)\right\} & =J_{i}(q), \\
D\left\{\phi_{i}(p), g(p, q)\right\} & =J_{i}(q), \\
D\left\{\phi_{i}(p), n(p, q)\right\} & =\phi_{i}(q)-J_{i}(q) .
\end{aligned}
$$


We observe here again that the integrals which combine a solution of a Dirichlet problem and a solution of a Neumann problem are purely geometric expressions.

Adding now (3) and (5) and using the definition 2.8 (2), we obtain

$$
D\left\{w_{i}(p), n(p, q)\right\}=\phi_{i}(q) / 2 .
$$

Thus, if we assume for fixed $q$ and $p$ near infinity the expansion

$$
n(p, q)=r^{-3} \sum_{i=1}^{3} b_{i}(q) x_{i}+O\left(r^{-3}\right)
$$

we find in view of (6) and $2.8(4)$ :

$$
b_{i}(q)=\frac{\phi_{i}(q)}{4 \pi} .
$$

This result shows that $\phi_{i}(q)$ may be obtained from $n(p, q)$ by expanding the latter function into a series in $p$ and taking the coefficient of $x_{i} r^{-3}$. We may express this result also in the following form:

$$
N(p, q)=\frac{1}{4 \pi r}+\frac{1}{4 \pi r^{3}} \sum_{i=1}^{3} \phi_{i}(q) x_{i}+O\left(r^{3}\right) .
$$

Adding similarly (1) and (4), we obtain

$$
D\left\{w_{i}(p), g(p, q)\right\}=\psi_{i}(q) / 2 .
$$

Let us now expand $g(p, q)$ for fixed $q$, and $p$ near infinity. In view of 2.4 (13),

$$
g(p, q)=\frac{u(q)}{4 \pi r}+\frac{1}{r^{3}} \sum_{i=1}^{3} c_{i}(q) x_{i}+O\left(r^{-3}\right)
$$

Applying 2.8 (4) and (10), we find

$$
-\alpha_{i} u(q)+4 \pi c_{i}(q)=\psi_{i}(q) \text {. }
$$

Since $u(q)$ may be obtained from the expansion of $g(p, q)$ at infinity and $\alpha_{i}$ from the expansion of $u(q)$ at infinity, we see that $\psi_{i}(q)$ is entirely determined by the coefficients of the expansion of Green's function.

It is apparent from these results that the problems of the polarization and virtual mass dipole are essentially contained in the corresponding problems of a single source and may be derived from them. It is, however, in most cases easier and more elegant to solve the dipole problem directly. One obtains also a much clearer understanding of the theoretical properties of the functions $\psi_{i}$ and $\phi_{i}$ if one starts with their definition by boundary conditions instead of defining them as coefficients of certain expansions. This is the way we shall proceed in the special cases treated in Chapter V.

Using Schwarz's inequality in (3) and (4), we find by virtue of 1.5 (16), 
$1.5(17)$, and $2.3(5), 2.3(10)$ :

$$
\begin{aligned}
& \left(J_{i}(q)\right)^{2} \leqq\left(4 \pi e_{i i}-V\right) n(q, q), \\
& \left(J_{i}(q)\right)^{2} \leqq\left(4 \pi d_{i i}-V\right) g(q, q) .
\end{aligned}
$$

From (1) and (5) we derive in the same way:

$$
\begin{aligned}
& \left(\psi_{i}(q)-J_{i}(q)\right)^{2} \leqq\left(4 \pi e_{i i}-V\right) g(q, q), \\
& \left(\phi_{i}(q)-J_{i}(q)\right)^{2} \leqq\left(4 \pi d_{i i}-V\right) n(q, q) .
\end{aligned}
$$

All these inequalities lead to estimates for certain potential theoretic constants if other constants of the same kind are known.

2.10. Inequalities. From our previous identities for Dirichlet integrals we may derive inequalities by the following general method. We construct linear combinations of harmonic functions of the class $H$. Expressing the fact that the norm of each function is non-negative leads to certain inequalities between Dirichlet integrals and, by virtue of the identities mentioned, between the harmonic functions and their coefficients.

We take, for example, the combination

$$
h=\sum_{i=1}^{3} t_{i} \phi_{i}+\tau \sum_{i=1}^{3} t_{i}^{\prime} \psi_{i}, \quad \sum_{i=1}^{3} t_{i}^{2}=\sum_{i=1}^{3}{t_{i}^{\prime 2}}^{2}=1 .
$$

Since, because of the table in 1.5 ,

$$
\begin{aligned}
D(h)= & \left(4 \pi \sum_{i, k=1,2,3} d_{i k} t_{i} t_{k}-V\right)+2 \tau \sum_{i=1}^{3} t_{i} t_{i}^{\prime} V \\
& +\tau^{2}\left(4 \pi \sum_{i, k=1,2,3} e_{i k} t_{i}^{\prime} t_{k}^{\prime}-V\right)
\end{aligned}
$$

we find the discriminant condition

$$
\left(\sum_{i=1}^{3} t_{i} t_{i}^{\prime}\right)^{2} V^{2} \leqq\left(4 \pi \sum_{i, k=1,2,3} d_{i k} t_{i} t_{k}-V\right)\left(4 \pi \sum_{i, k=1,2,3} e_{i k} t_{i}^{\prime} t_{k}^{\prime}-V\right) .
$$

This is a generalization of the inequality $W P \geqq V^{2}$ announced in 1.5.

We may further consider the following function of class $H$ :

$$
h(p)=\sum_{i=1}^{3} t_{i} \psi_{i}(p)+\tau v(p), \quad \sum_{i=1}^{3} t_{i}^{2}=1 .
$$

We have because of 2.5 (2)

$$
D\left(\psi_{i}, v\right)=\iint_{b}\left(\alpha_{i}-x_{i}\right) d \sigma=S\left(\alpha_{i}-\eta_{i}\right)
$$

where $S$ is the surface area of $b$ and 


$$
\eta_{i}=S^{-1} \iint_{b} x_{i} d \sigma
$$

is the $i$ th coordinate of the center of gravity of a homogeneous mass distribution on $b$. We remember that $\alpha_{i}$ was the $i$ th coordinate of the centroid of an equilibrium charge distribution on the conductor $b$. The terms $\alpha_{i}-\eta_{i}$ measure, therefore, the deviation between the electrostatic and the material centroid of $b$. We have

$$
D(h)=\left(4 \pi \sum_{i, k=1,2,3} e_{i k} t_{i} t_{k}-V\right)+2 \tau S \sum_{i=1}^{3} t_{i}\left(\alpha_{i}-\eta_{i}\right)+\tau^{2} \frac{\Gamma}{4 \pi} .
$$

Hence, we have the discriminant condition

$$
4 \pi S^{2}\left(\sum_{i=1}^{3} t_{i}\left(\alpha_{i}-\eta_{i}\right)\right)^{2} \leqq \Gamma\left(4 \pi \sum_{i, k=1,2,3} e_{i k} t_{i} t_{k}-V\right)=\Gamma P .
$$

We construct next the combination

$$
h(p)=\sum_{i=1}^{3} t_{i} \psi_{i}(p)+\sum_{\nu=1}^{m} t_{\nu}^{\prime} n\left(p, q_{\nu}\right), \quad q_{\nu} \in B, \quad \sum_{i=1}^{3} t_{i}^{2}=1 .
$$

We are led to the inequality

$$
\left(\sum_{i=1}^{3} \sum_{\nu=1}^{m} t_{i} t_{\nu}^{\prime} J_{i}\left(q_{\nu}\right)\right)^{2} \leqq P \sum_{p, \mu=1}^{m}\left(n\left(q_{v}, q_{\mu}\right)-I\left(q_{\nu}, q_{\mu}\right)\right) t_{\nu}^{\prime} t_{\mu}^{\prime} .
$$

In exactly the same way we find

$$
\left(\sum_{i=1}^{3} \sum_{\nu=1}^{m} t_{i} t_{\nu}^{\prime} J_{i}\left(q_{\nu}\right)\right)^{2} \leqq W \sum_{\nu, \mu=1}^{m}\left(g\left(q_{\nu}, q_{\mu}\right)-I\left(q_{\nu}, q_{\mu}\right)\right) t_{\nu}^{\prime} t_{\mu}^{\prime} .
$$

We have in (10) and (11) interesting relationships between two quadratic forms, the one being related to a Dirichlet problem, the other to a Neumann problem. Both forms are known to be positive-definite. Their product appears to be estimated by means of a purely geometric lower bound. Thus, if the one quadratic form becomes very small, the other one has to be large. The inequalities (3) and 2.3 (17) are of the same type.

We obtain easily further inequalities of similar kind; consider, for example, the function

$$
h(p)=\sum_{i=1}^{3} t_{i} \phi_{i}(p)+\tau\left(\frac{1}{r(p, o)}-\frac{u(p)}{C}\right), \quad o \in \widetilde{B}, \quad \sum_{i=1}^{3} t_{i}^{2}=1 .
$$

We find

$$
D\left\{\phi_{i}, \frac{1}{r(p, o)}\right\}=\iint_{b} \frac{1}{r(p, o)} \frac{\partial x_{i}}{\partial \nu} d \sigma=j_{i}(o)
$$


where $j_{i}(o)$ is a quantity depending on the shape of $b$ and the point of reference 0 . Thus

$$
\left(\sum_{i=1}^{3} t_{i} j_{i}(o)\right)^{2} \leqq W \cdot\left(D\left(r^{-1}\right)-\frac{4 \pi}{C}\right)
$$

follows as discriminant condition for the quadratic form $D(h)$. The second factor on the right is positive, in view of 2.4 (9), for every choice of the point of reference $o \in \widetilde{B}$. This fact is coupled here with the virtual mass into one inequality of the above mentioned type.

If we consider

$$
D\left\{\psi_{i}, \frac{1}{r(p, o)}\right\}=-\iint_{b}\left(x_{i}-\alpha_{i}\right) \frac{\partial}{\partial \nu}\left(\frac{1}{r(p, o)}\right) d \sigma=-4 \pi \alpha_{i}+j_{i}^{*}(o)
$$

where $j^{*}(o)$ is again a simple geometric measure of $b$, we find by the above reasoning

$$
\left(\sum_{i=1}^{3} t_{i}\left(j_{i}^{*}(o)-4 \pi \alpha_{i}\right)\right)^{2} \leqq P\left(D\left(r^{-1}\right)-\frac{4 \pi}{C}\right) .
$$

This inequality is not as important as (14) since in this case the terms $\alpha_{i}$, which are not of geometric but of potential theoretic nature, appear in the left.

\section{Chapter III. Inequalities}

The greater part of this chapter is devoted to inequalities for the virtual mass. The starting point is a characterization of the virtual mass $W$ in any given direction $h$ as a minimum and a maximum of certain integrals. These characterizations correspond to the principles of Dirichlet and Thomson for the electrostatic capacity. They are essentially the adaptations of well known results to this particular problem.

Then, choosing the arbitrary quantities involved in these integrals, in an appropriate way, lower bounds will be obtained for the virtual mass $W$ and for the average virtual mass $W_{m}$.

In a further course of this chapter the monotonic character of the dipole forms $D$ and $E[1.2(1)]$ is proved. The method used will have some importance for the problems dealt with in Chapter IV.

3.1. Characterization of the virtual mass. (a) $W$ is a maximum. We have (cf. Diaz-Weinstein [4])

$$
W=\max \frac{\left(\iint f h \cdot \nu d \sigma\right)^{2}}{\iiint|\operatorname{grad} f|^{2} d \tau}
$$

where $f$ is an arbitrary function defined in $B$, that is, outside of the given surface $b ; \nu$ is the exterior normal, $d \sigma$ the surface element of $b, d \tau$ the volume 
element of $B$. The maximum is attained if $f=\phi$ where $\phi \in H_{0}$ is the velocity potential corresponding to the given direction $h$. For this function

$$
\nabla^{2} \phi=0, \quad-\frac{\partial \phi}{\partial \nu}=h \cdot \nu \text { on } b, \quad \phi=O\left(r^{-2}\right) \text { as } r \rightarrow \infty
$$

so that

$$
\iiint|\operatorname{grad} \phi|^{2} d \tau=-\iint \phi \frac{\partial \phi}{\partial \nu} d \sigma=\iint \phi \boldsymbol{h} \cdot \nu d \sigma=W .
$$

The maximum property (1) follows by Green's identity and Schwarz's inequality:

(4)

$$
\begin{aligned}
\iint f \boldsymbol{h} \cdot \nu d \sigma= & -\iint f \frac{\partial \phi}{\partial \nu} d \sigma=\iiint \operatorname{grad} f \cdot \operatorname{grad} \phi d \tau \\
& +\iiint f \nabla^{2} \phi d \tau=\iiint \operatorname{grad} f \cdot \operatorname{grad} \phi d \tau \\
\left(\iint f \boldsymbol{h} \cdot \nu d \sigma\right)^{2} \leqq & \iiint|\operatorname{grad} f|^{2} d \tau \cdot \iiint|\operatorname{grad} \phi|^{2} d \tau \\
= & \iiint|\operatorname{grad} f|^{2} d \tau \frac{\left(\iint \phi \boldsymbol{h} \cdot \nu d \sigma\right)^{2}}{\iiint|\operatorname{grad} \phi|^{2} d \tau} .
\end{aligned}
$$

(b) $W$ is a minimum. Let $\boldsymbol{q}$ be an arbitrary vector function defined outside of the solid, satisfying the conditions:

$$
\begin{aligned}
\operatorname{div} \boldsymbol{q}=0 & & \text { in } B, \\
-\boldsymbol{q} \cdot \boldsymbol{\nu}=\boldsymbol{h} \cdot \boldsymbol{\nu} & & \text { on } b .
\end{aligned}
$$

The meaning of the first condition is that the vector-field $q$ is sourceless. We have then

$$
W=\min \iiint|q|^{2} d \tau
$$

where the integration is extended over $B$. The minimum is attained if $q=\operatorname{grad} \phi$. In this case conditions (5) are indeed satisfied and the integral in (6) reduces to $W$. The minimum property (6) follows from

$$
\begin{aligned}
\iiint|q-\operatorname{grad} \phi|^{2} d \tau & \\
& =\iiint|q|^{2} d \tau+W-2 \iiint q \cdot \operatorname{grad} \phi d \tau \geqq 0 ;
\end{aligned}
$$

the last integral equals, by (5) and by Green's identity, 


$$
\iiint \operatorname{div}(\boldsymbol{q} \phi) d \tau=-\iint \boldsymbol{q} \cdot \boldsymbol{\nu} \boldsymbol{\phi} d \sigma=\iint \boldsymbol{h} \cdot \boldsymbol{\nu} \phi d \sigma=W
$$

An important additional remark is the following. The minimum property in question holds good allowing a slightly more general class of vector functions $\boldsymbol{q}$. They satisfy (5) except along a (closed or open) surface in the field along which $\boldsymbol{q}$ and $\boldsymbol{q} \cdot \boldsymbol{\nu}$ must have one-sided limits and $\boldsymbol{q} \cdot \boldsymbol{\nu}$ must be continuous.

The proof follows without difficulty by the previous argument.

3.2. Characterization of the polarization. For the sake of comparison we mention a well known characterization of the polarization as a minimum. This is the following:

$$
P=\min \iint|\operatorname{grad} f|^{2} d \tau ; \quad f=\boldsymbol{h} \cdot \boldsymbol{r}+\text { const. on } b .
$$

Indeed, the integral in (1) can be written as follows:

$$
\begin{aligned}
\iiint|\operatorname{grad}(f-\psi)|^{2} d \tau & +\iiint|\operatorname{grad} \psi|^{2} d \tau \\
& +2 \iiint \operatorname{grad}(f-\psi) \cdot \operatorname{grad} \psi d \tau
\end{aligned}
$$

and the last integral is zero since $f-\psi=$ const. on the surface.

3.3. Another maximum property of the virtual mass. A slightly different form of the maximum property discussed in 3.1 (a) is the following:

$$
W \geqq 2 \iint f h \cdot \nu d \sigma-\iiint|\operatorname{grad} f|^{2} d \tau,
$$

the virtual mass $W$ being the maximum of the expression on the right-hand side; here $f$ is again arbitrary. Indeed, the ratio occurring in 3.1 (1) is never less than the right-hand expression in (1). On the other hand (1) becomes an equation if $f=\phi$.

3.4. Lower bounds for the virtual mass. (a) We specialize the function $f$ occurring in 3.3 (1) as follows: $f=\boldsymbol{h} \cdot \boldsymbol{r} g$ where $g=g_{0}=$ const. on the surface $b$ of the given solid. Then

$$
\begin{aligned}
W \geqq & 2 g_{0} \iint(\boldsymbol{h} \cdot \boldsymbol{r})(\boldsymbol{h} \cdot \boldsymbol{\nu}) d \sigma \\
& -\iiint|\boldsymbol{h} \cdot \boldsymbol{r} \operatorname{grad} g+g \operatorname{grad}(\boldsymbol{h} \cdot \boldsymbol{r})|^{2} d \tau .
\end{aligned}
$$

The first integral is the volume $V$ of the solid bounded by $b$. The second can be written as follows: 


$$
\begin{aligned}
& \iiint\left\{(\boldsymbol{h} \cdot \boldsymbol{r})^{2}|\operatorname{grad} g|^{2}+g^{2}|\operatorname{grad}(\boldsymbol{h} \cdot \boldsymbol{r})|^{2}\right. \\
&+2 g(\boldsymbol{h} \cdot \boldsymbol{r}) \operatorname{grad} g \cdot \operatorname{grad}(\boldsymbol{h} \cdot \boldsymbol{r})\} d \tau \\
&= \iiint\left\{(\boldsymbol{h} \cdot \boldsymbol{r})^{2}|\operatorname{grad} g|^{2}+g^{2}\right\} d \tau \\
&+\frac{1}{2} \iiint \operatorname{grad}\left(g^{2}\right) \cdot \operatorname{grad}\left((\boldsymbol{h} \cdot \boldsymbol{r})^{2}\right) d \tau \\
&= \iiint\left\{(\boldsymbol{h} \cdot \boldsymbol{r})^{2}|\operatorname{grad} g|^{2}+g^{2}\right\} d \tau \\
&-\frac{1}{2} \iiint g^{2} \nabla^{2}\left((\boldsymbol{h} \cdot \boldsymbol{r})^{2}\right) d \tau-\frac{1}{2} \iint g^{2} \frac{\partial(\boldsymbol{h} \cdot \boldsymbol{r})^{2}}{\partial \nu} d \sigma \\
&= \iiint(\boldsymbol{h} \cdot \boldsymbol{r})^{2}|\operatorname{grad} g|^{2} d \tau-g_{0}^{2} V .
\end{aligned}
$$

Hence

$$
W \geqq 2 g_{0} V+g_{0}^{2} V-\iiint(\boldsymbol{h} \cdot \boldsymbol{r})^{2}|\operatorname{grad} g|^{2} d \tau .
$$

In particular

$$
W_{m} \geqq 2 g_{0} V+g_{0}^{2} V-\frac{1}{3} \iiint r^{2}|\operatorname{grad} g|^{2} d \tau .
$$

The previous deduction shows that the difference (2) is always non-negative so that the lower bound in (3) and (4) is never greater than $2 g_{0} V$.

Replacing $g$ by $k g$ and determining $k$ in the most advantageous way we find the following alternate forms for (3) and (4):

$$
\begin{gathered}
W \geqq \frac{g_{0}^{2} V^{2}}{\iiint(\boldsymbol{h} \cdot \boldsymbol{r})^{2}|\operatorname{grad} g|^{2} d \tau-g_{0}^{2} V}, \\
W_{m} \geqq \frac{g_{0}^{2} V^{2}}{(1 / 3) \iiint r^{2}|\operatorname{grad} g|^{2} d \tau-g_{0}^{2} V} .
\end{gathered}
$$

(b) $\left(^{6}\right)$ Let $b_{\alpha}$ be an arbitrary family of closed surfaces, $0<\alpha<\infty$, chosen in such a way that $b_{0}$ coincides with the surface $b, b_{\alpha}$ contains $b_{\beta}$ if $\alpha>\beta$, and $b_{\alpha}$ will contain an arbitrary sphere provided $\alpha$ is sufficiently large. We choose the function $g$ as follows: $g=g(\alpha)$ so that $g$ is constant on $b_{\alpha}, g(0)=g_{0}$; moreover we assume that $g(\infty)=0$.

(8) Concerning the following argument, cf. Pólya-Szegö $[16,2.5]$. 
Let us compute the contribution of the infinitesimal domain between $b_{\alpha}$ and $b_{\alpha+d \alpha}$ to the integral in (6), $d \alpha>0$; we have

$$
d \tau=d \sigma \frac{d \alpha}{|\operatorname{grad} \alpha|} .
$$

Introducing the integral

$$
T(\alpha)=\iint_{b_{\alpha}} r^{2}|\operatorname{grad} \alpha| d \sigma
$$

we obtain for this contribution: $T(\alpha)\left(g^{\prime}(\alpha)\right)^{2} d \alpha$ so that we find

$$
\iiint r^{2}|\operatorname{grad} g|^{2} d \tau=\int_{0}^{\infty} T(\alpha)\left(g^{\prime}(\alpha)\right)^{2} d \alpha .
$$

By Schwarz's inequality

$$
\int_{0}^{\infty} T(\alpha)\left(g^{\prime}(\alpha)\right)^{2} d \alpha \cdot \int_{0}^{\infty} T(\alpha)^{-1} d \alpha \geqq\left(\int_{0}^{\infty} g^{\prime}(\alpha) d \alpha\right)^{2}=g_{0}^{2}
$$

Introducing the notation

$$
I=\int_{0}^{\infty}(T(\alpha))^{-1} d \alpha
$$

we obtain that the quantity in (9) is a minimum if we choose $g^{\prime}(\alpha)$ $=$ const. $T(\alpha)^{-1}$, that is,

$$
g(\alpha)=\frac{g_{0}}{I} \int_{\alpha}^{\infty}(T(\alpha))^{-1} d \alpha .
$$

The minimum value of (9) will be $g_{0}^{2} / I$ so that

$$
W_{m} \geqq \frac{V^{2}}{(3 I)^{-1}-V} \text {. }
$$

We observe that the function $T(\alpha)$ and the integral $I$ depend on the choice of the surfaces $b_{\alpha}$. Once these surfaces are chosen the bound (13) holds.

In case of a sphere $r=a$ we may choose for $b_{a}$ the concentric spheres $r=a+\alpha$ and obtain

$$
T(\alpha)=4 \pi(a+\alpha)^{4}, \quad I=\left(12 \pi a^{3}\right)^{-1}
$$

so that in (13) the equation will hold.

A similar argument furnishes the bound

$$
W \geqq \frac{V^{2}}{\left(3 I_{h}\right)^{-1}-V}
$$


where

$$
I_{h}=\int_{0}^{\infty}\left(T_{h}(\alpha)\right)^{-1} d \alpha, \quad T_{h}(\alpha)=3 \iint_{b_{\alpha}}(\boldsymbol{h} \cdot \boldsymbol{r})^{2}|\operatorname{grad} \alpha| d \sigma .
$$

Inequality (15) becomes an equation for an ellipsoid provided the vector $h$ points in the direction of one of the principal axes and the $b_{\alpha}$ are the confocal ellipsoids.

3.5. First application: proof of 1.7 (3). Let us assume that the given surface $b$ is star-shaped with respect to a certain interior point which we choose as the origin of the coordinate system. We specialize the function $f$ of 3.3 (1) as follows: $f=k \boldsymbol{h} \cdot \boldsymbol{r} r^{-3}$ where $k$ is a constant. Denoting the values of $r$ which correspond to the points of the surface $b$ by $r_{0}$ we have

$$
\begin{aligned}
\iint f \boldsymbol{h} \cdot \nu d \sigma & =k \iint(\boldsymbol{h} \cdot \boldsymbol{r})(\boldsymbol{h} \cdot \boldsymbol{\nu}) \boldsymbol{r}_{0}^{-3} d \sigma \\
\iiint|\operatorname{grad} f|^{2} d \tau & =k^{2} \iiint\left|\boldsymbol{h} \boldsymbol{r}^{-3}-3(\boldsymbol{h} \cdot \boldsymbol{r}) \boldsymbol{r} \boldsymbol{r}^{-5}\right|^{2} d \tau \\
& =k^{2} \iiint \boldsymbol{r}^{-6}\left(1+3(h \cdot t)^{2}\right) d \tau
\end{aligned}
$$

where $t$ is the unit vector $r / r$. Introducing polar coordinates we have $d \tau=r^{2}$ $\cdot d r \cdot d \omega$ where $d \omega$ is the surface element of the unit sphere, so that integrating with respect to $r$, we have

$$
W \geqq 2 k \iint(\boldsymbol{h} \cdot \boldsymbol{r})(\boldsymbol{h} \cdot \nu) r_{0}^{-3} d \sigma-\frac{1}{3} k^{2} \iint r_{0}^{-3}\left(1+3(\boldsymbol{h} \cdot \boldsymbol{t})^{2}\right) d \omega .
$$

Hence

$$
W_{m} \geqq \frac{2}{3} k \iint \boldsymbol{r} \cdot \nu r_{0}^{-3} d \sigma-\frac{2}{3} k^{2} \iint r_{0}^{-3} d \omega
$$

But on $b$ :

$$
\boldsymbol{r} \cdot \nu d \sigma=r_{0} \cos (r, \nu) d \sigma=r_{0}^{3} d \omega
$$

so that

$$
W_{m} \geqq \frac{8 \pi}{3} k-\frac{2}{3} k^{2} \iint r_{0}^{-3} d \omega .
$$

Choosing $k$ properly we obtain

$$
W_{m} \geqq \frac{8 \pi^{2}}{3}\left(\iint r_{0}^{-8} d \omega\right)^{-1}
$$


which is equivalent to 1.7 (3).

3.6. Second application: proof of 1.7 (5). We consider again a surface $b$ which is star-shaped with respect to the origin. We choose for the surfaces $b_{\alpha}$ in 3.4 (b) the surfaces similar to the given one with respect to the origin. The given surface is $b=b_{0}$. Let $r=r_{0}$ be the equation of $b_{0}$ in polar coordinates; then the equation of $b_{\alpha}$ will be $r=r_{0}(1+\alpha)$. As is well known,

$$
\begin{aligned}
d \sigma & =r^{2} \sin \theta\left\{1+r^{-2}\left(\left(\frac{\partial r}{\partial \theta}\right)^{2}+\frac{1}{\sin ^{2} \theta}\left(\frac{\partial r}{\partial \phi}\right)^{2}\right)\right\}^{1 / 2} d \theta d \phi \\
& =r^{2}\left\{1+r^{-2} \nabla(r)\right\}^{1 / 2} d \omega \\
& =(1+\alpha)^{2} r_{0}^{2}\left\{1+r_{0}^{-2} \nabla\left(r_{0}\right)\right\}^{1 / 2} d \omega,
\end{aligned}
$$

where $\nabla$ denotes Beltrami's operator $(1.7(5))$. For any scalar function $f=f(r, \theta, \phi)$ we have

$$
|\operatorname{grad} f|^{2}=\left(\frac{\partial f}{\partial r}\right)^{2}+\frac{1}{r^{2}} \nabla(f)
$$

so that

$$
|\operatorname{grad} \alpha|^{2}=r_{0}^{-2}+\nabla\left(r_{0}^{-1}\right)=r_{0}^{-2}+r_{0}^{-4} \nabla\left(r_{0}\right) .
$$

Consequently, in view of (1),

$$
T(\alpha)=(1+\alpha)^{4} \iint r_{0}^{3}\left(1+r_{0}^{-2} \nabla\left(r_{0}\right)\right) d \omega=(1+\alpha)^{4} T(0)
$$

where

$$
T(0)=3 V+\iint r_{0} \nabla\left(r_{0}\right) d \omega=3 V+\frac{4}{9} \iint \nabla\left(r_{0}^{3 / 2}\right) d \omega
$$

Hence

$$
I=\int_{0}^{\infty}\left((1+\alpha)^{4} T(0)\right)^{-1} d \alpha=(3 T(0))^{-1} .
$$

Now we use $3.4(13)$ and obtain $1.7(5)$.

In case of a sphere $r_{0}=$ const., $\nabla=0$, and the inequality in question becomes an equation.

3.7. Virtual mass, polarization and volume. Finally, we return to the elegant inequality $P W \geqq V^{2}$ which is a special case of 2.10 (3). In all the cases when $P$ is known (or an upper bound for $P$ is known) this inequality furnishes a lower bound for the virtual mass. In view of this and of the simplicity of the inequality, it is worth while to discuss it directly.

(a) We choose in 3.1 (1): $f=\psi$ where $\psi$ is the electric potential satisfying the condition $\psi=\boldsymbol{h} \cdot \boldsymbol{r}+$ const on the surface $b$. Then 


$$
\iint f \boldsymbol{h} \cdot \nu d \sigma=\iint(\boldsymbol{h} \cdot \boldsymbol{r})(\boldsymbol{h} \cdot \nu) d \sigma=V, \quad \iiint|\operatorname{grad} f|^{2} d \tau=P,
$$

so that the inequality follows.

(b) Let $\alpha$ be a parameter, $\phi$ and $\psi$ the velocity and electric potential, respectively, corresponding to the direction $h$. Then

$$
\begin{aligned}
\iiint|\operatorname{grad} \phi+\alpha \operatorname{grad} \psi|^{2} d \tau & \\
= & W+P \alpha^{2}+2 \alpha \iiint \operatorname{grad} \phi \cdot \operatorname{grad} \psi d \tau .
\end{aligned}
$$

The last integral can be written as follows:

$$
-\iint \psi \frac{\partial \phi}{\partial \nu} d \sigma=\iint(\boldsymbol{h} \cdot \boldsymbol{r}) \cdot(\boldsymbol{h} \cdot \nu) d \sigma=V
$$

so that

$$
W+P \alpha^{2}+2 V \alpha \geqq 0 .
$$

This implies the inequality in question.

Multiplying the inequality

$$
W \geqq V^{2} / P
$$

by the surface element $d \omega$ of the unit sphere $h_{1}, h_{2}, h_{3}$ and integrating we obtain

$$
W_{m} \geqq \frac{V^{2}}{4 \pi} \iint \frac{d \omega}{P} .
$$

By an appropriate rotation the last integral can be transformed into

$$
\iint \frac{d \omega}{P_{1} h_{1}^{2}+P_{2} h_{2}^{2}+P_{3} h_{3}^{2}}
$$

where $P_{1}, P_{2}, P_{3}$ are the characteristic values of the form $P$. This integral is not less than

$$
\begin{aligned}
\left\{\iint\left(P_{1} h_{1}^{2}+P_{2} h_{2}^{2}+P_{3} h_{3}^{2}\right) d \omega\right\}^{-1} & \left(\iint d \omega\right)^{2} \\
= & \left\{\left(P_{1}+P_{2}+P_{3}\right) / 3\right\}^{-1} \cdot 4 \pi=P_{m}^{-1} \cdot 4 \pi
\end{aligned}
$$

from which the inequality 1.8 (1) for the average virtual mass and average polarization follows.

3.8. Monotony of the dipole forms $D$ and $E$. (a) Let us consider a solid contained in the interior of another one. We denote the solids and at the same 
time their volumes by $V$ and $V^{\prime}\left(V\right.$ is in $V^{\prime}$ ), the corresponding virtual masses by $W$ and $W^{\prime}$, the polarizations by $P$ and $P^{\prime}$, the velocity potentials by $\phi$ and $\phi^{\prime}$, and the electric potentials by $\psi$ and $\psi^{\prime}$. We have by 3.1 (b)

$$
W=\min \iiint|q|^{2} d \tau
$$

where

$$
\operatorname{div} \boldsymbol{q}=0, \quad-\boldsymbol{q} \cdot \boldsymbol{\nu}=\boldsymbol{h} \cdot \boldsymbol{\nu} .
$$

According to the remark made at the end of that section we may allow the discontinuity of $\boldsymbol{q}$ along a surface provided the one-sided limits of $\boldsymbol{q}$ and $\boldsymbol{q} \cdot \boldsymbol{\nu}$ exist and $\boldsymbol{q} \cdot \boldsymbol{\nu}$ is continuous.

We choose now $q=h$ in $V^{\prime}-V$ and $q=\operatorname{grad} \phi^{\prime}$ outside of the larger solid $V^{\prime}$. Then the conditions mentioned are satisfied and we obtain

$$
W \leqq \iiint_{V^{\prime}-V}|h|^{2} d \tau+\iiint\left|\operatorname{grad} \phi^{\prime}\right|^{2} d \tau=V^{\prime}-V+W^{\prime}
$$

so that $W+V \leqq W^{\prime}+V^{\prime}$. This establishes the assertion.

(b) In the minimum assertion of 3.2 we choose: $f=(\boldsymbol{h} \cdot \boldsymbol{r})+$ const. in $V^{\prime}-V$ and $f=\psi^{\prime}$ outside of the larger solid. This is a continuous function (with a possible discontinuity of $\partial f / \partial \nu$ along the boundary surface of $\left.V^{\prime}\right)$. We have

(4) $\quad P \leqq \iiint_{V^{\prime}-V}|\operatorname{grad}(\boldsymbol{h} \cdot \boldsymbol{r})|^{2} d \tau+\iiint\left|\operatorname{grad} \psi^{\prime}\right|^{2} d \tau=V^{\prime}-V+P^{\prime}$,

so that $P+V \leqq P^{\prime}+V^{\prime}$ follows.

Inequalities 1.9 (1) follow from this result immediately.

\section{Chapter IV. Variational Results}

All potential theoretic quantities considered in this paper are functionals of the boundary surface $b$ of the domain $B$. It is of interest to study their dependence on this surface and, in particular, to establish formulas for their variation under infinitesimal deformations of $b$. Quantities which appear by these formulas as monotonically increasing with expanding surface $b$ will be of particular interest since they can easily be estimated by use of smaller and larger domains of comparison with respect to $B$. Examples of such quantities are the dipole forms $D$ and $E$ as proved in 3.8. It is of ten possible to estimate terms which are not monotonic in the above sense by combining them with other elements into a monotonic aggregate which may easily be estimated. Finally, variational formulas have a great heuristic value to arrive at possible identities and inequalities between different functionals in potential theory. Conjectured inequalities must remain true if the domain $B$ is arbitrarily varied and this condition leads to a severe check on possible inequalities. 
Using certain identities for Dirichlet integrals derived in Chapter II we obtain at first formulas holding for a finite deformation of $b$. Applying them to infinitesimal variations they become simpler and easier applicable. The method used in 4.1 and 4.2 is based on a similar argument as used in 3.8.

4.1. Variation of Green's function. (a) Let $b$ be, as before, the smooth boundary of the domain $B$; let $b^{\prime}$ be a surface of the same character which encloses $b$ and let $B^{\prime}$ be the infinite domain bounded by $b^{\prime}$. The surfaces $b$ and $b^{\prime}$ enclose a shell domain $\Sigma$ such that $B=\Sigma+B^{\prime}$. We denote Green's function for $B$ again by $G(p, q)$, and if $p$ and $q$ lie both in $B^{\prime}$ we denote by $G^{\prime}(p, q)$ Green's function for $B^{\prime}$. In general, we shall denote corresponding terms with respect to $B$ and $B^{\prime}$ by the same letter and distinguish those of $B^{\prime}$ by a dash. Thus $D(\lambda, \mu)$ denotes the Dirichlet integral taken over the domain $B$ while $D^{\prime}(\lambda, \mu)$ is the integral with the same integrand but extended over $B^{\prime}$ only.

We define in $B$ the continuous function:

$$
g^{*}(p, q)=\frac{1}{4 \pi r(p, q)} \text { in } \Sigma, \quad g^{*}(p, q)=g^{\prime}(p, q) \text { in } B^{\prime}, \quad q \text { fixed in } B^{\prime} .
$$

Let $q$ and $s$ be points in $B^{\prime}$ and consider the identity

$$
\begin{aligned}
D\left\{g^{*}(p, q), g^{*}(p, s)\right\}= & D\{g(p, q), g(p, s)\} \\
& +D\left\{g(p, q), g^{*}(p, s)-g(p, s)\right\} \\
& +D\left\{g^{*}(p, q)-g(p, q), g(p, s)\right\} \\
& +D\left\{g^{*}(p, q)-g(p, q), g^{*}(p, s)-g(p, s)\right\} .
\end{aligned}
$$

Since $g^{*}$ is continuous on $b^{\prime}$ and $g$ is continuously differentiable there, we have by Green's identity

$$
\begin{aligned}
D\left\{g(p, q), g^{*}(p, s)-g(p, s)\right\} & \\
& =-\iint_{b}\left(\frac{1}{4 \pi r(p, s)}-g(p, s)\right) \frac{\partial}{\partial \nu} g(p, q) d \sigma=0 .
\end{aligned}
$$

Similarly

$$
D\left\{g^{*}(p, q)-g(p, q), g(p, s)\right\}=0 .
$$

We have, further, in view of definition (1):

$$
\begin{aligned}
& D\left\{g^{*}(p, q), g^{*}(p, s)\right\} \\
& \quad=\frac{1}{16 \pi^{2}} \iiint_{\Sigma} \operatorname{grad} \frac{1}{r(p, q)} \cdot \operatorname{grad} \frac{1}{r(p, s)} d \tau+D^{\prime}\left\{g^{\prime}(p, q), g^{\prime}(p, s)\right\} .
\end{aligned}
$$

Using the integral $I(q, s)$ of 2.3 (4) and denoting by $I^{\prime}(q, s)$ the integral with the same integrand extended over the complement $\widetilde{B}^{\prime}$ of $B^{\prime}$, we may 
write

$$
\frac{1}{16 \pi^{2}} \iiint_{\Sigma} \operatorname{grad} \frac{1}{r(p, q)} \cdot \operatorname{grad} \frac{1}{r(p, s)} d \tau=I^{\prime}(q, s)-I(q, s) .
$$

Now we apply the identity $2.3(5)$ and the corresponding identity with respect to $B^{\prime}$. In view of (3), (4), (5), and (6), we may bring (2) into the form

$$
g^{\prime}(q, s)=g(q, s)+D\left\{g^{*}(p, q)-g(p, q), g^{*}(p, s)-g(p, s)\right\} .
$$

We note further that for $p \in \Sigma$ the identity $g^{*}(p, q)-g(p, q)=G(p, q)$ holds. Hence (7) may be put into the final form

$$
\begin{aligned}
G^{\prime}(q, s)= & G(q, s)-\iiint_{\Sigma} \operatorname{grad} G(p, q) \cdot \operatorname{grad} G(p, s) d \tau_{p} \\
& -D^{\prime}\left\{G^{\prime}(p, q)-G(p, q), G^{\prime}(p, s)-G(p, s)\right\} .
\end{aligned}
$$

(b) Let now $q_{i}, i=1,2, \cdots, m$, be an arbitrary set of points in $B^{\prime}$ and $t_{i}, i=1,2, \cdots, m$, a set of real constants. Compare the two quadratic forms

$$
Q=\sum_{i, k=1}^{m} g\left(q_{i}, q_{k}\right) t_{i} t_{k} \text { and } Q^{\prime}=\sum_{i, k=1}^{m} g^{\prime}\left(q_{i}, q_{k}\right) t_{i} t_{k} .
$$

We have by virtue of $(8)$ :

$$
\begin{aligned}
Q^{\prime}=Q & +\iiint_{\Sigma}\left|\operatorname{grad} \sum_{i=1}^{m} t_{i} G\left(p, q_{i}\right)\right|^{2} d \tau \\
& +D^{\prime}\left\{\sum_{i=1}^{m} t_{i}\left(G^{\prime}\left(p, q_{i}\right)-G\left(p, q_{i}\right)\right)\right\} .
\end{aligned}
$$

Since $D^{\prime}(h) \geqq 0$, we have the estimate

$$
Q^{\prime} \geqq Q+\iiint_{\Sigma}\left|\operatorname{grad} \sum_{i=1}^{m} t_{i} G\left(p, q_{i}\right)\right|^{2} d \tau .
$$

Thus, we may formulate the theorem: The quadratic form $\sum_{i, k=1}^{m} g\left(q_{i}, q_{k}\right) t_{i} t_{k}$ increases monotonically for fixed $t_{i}$ and $q_{i}$ if the surface $b$ expands.

It may easily be seen that (11) contains the inequality $2.3(7)$ as a special case. In fact, if we keep the surface $b^{\prime}$ fixed and let the interior surface $b$ contract into a point, we see that $Q$ tends to zero, $G(p, q)$ to $(4 \pi r(p, q))^{-1}$, and the domain $\Sigma$ to $B^{\prime}$. Thus, we find in the limit

$$
Q^{\prime} \geqq \frac{1}{16 \pi^{2}} \iiint_{\widetilde{B}^{\prime}}\left|\operatorname{grad} \sum_{i=1}^{m} \frac{t_{i}}{r\left(p, q_{i}\right)}\right|^{2} d \tau
$$

which is equivalent to 2.3 (7).

(c) Let us return now to (8) and assume that $b$ and $b^{\prime}$ are in an $\epsilon$-neigh- 
borhood; that is, we suppose that the distance of each point on the one surface from the other is less than $\epsilon$. Then, it is well known that

$$
G^{\prime}(p, q)-G(p, q)=O(\epsilon) \quad \text { for } p, q \in B^{\prime},
$$

and this same estimate is valid for all derivatives of this difference. Hence, the term $D^{\prime}$ which occurs in (8) is of order $\epsilon^{2}$, while it is easily seen from geometric considerations that the first integral on the right-hand side of (8) is of order $\epsilon$. This fact makes the formula (8) particularly interesting, since it has just the form necessary to express $G^{\prime}(q, s)$ in terms of $G(q, s)$ in the form of a series in powers of $\epsilon$. In fact, we may use (8) as basic formula in a successive approximation of $G^{\prime}(q, s)$ by means of $G(q, s)$.

We may also derive from (8) a well known functional derivative equation for Green's function. We define on $b$ a continuously differentiable positive function $d(p)$; let $p^{\prime}$ on $b^{\prime}$ be obtained from $p \in b$ by proceeding from $p$ along the interior normal with respect to $B$ by an amount $\delta \nu=\epsilon d(p)$. This defines the surface $b^{\prime}$. If we want to apply formula (8) in this case, we may replace the first volume integral in the usual way by a surface integral over $b$ which contains $\delta \nu$; since the gradient of Green's function on $b$ has the direction of the interior normal, we may finally put (8) into the form:

$$
\delta G(q, s)=-\delta g(q, s)=-\iint_{b} \frac{\partial}{\partial \nu} G(p, q) \frac{\partial}{\partial \nu} G(p, s) \delta \nu d \sigma+O\left(\epsilon^{2}\right) .
$$

This formula for the variation of Green's function was given first by Hadamard [5] who made several applications of it. It may easily be extended to the case when $b^{\prime}$ does not necessarily enclose $b$ but lies in an arbitrary $\epsilon$-neighborhood of $b$.

The formula (8) is a finite extension of Hadamard's formula (14). Its importance lies in the fact that it permits the determination of higher than the first variation of Green's function. It is exceedingly difficult to obtain these variations directly from Hadamard's method. Our approach has also the advantage that it may be applied in exactly the same fashion to numerous other functionals.

4.2. Variation of Neumann's function. (a) In order to obtain a variational formula for Neumann's function, we define

$$
n^{*}(p, q)=-(4 \pi r(p, q))^{-1} \text { in } \Sigma, n^{*}(p, q)=n^{\prime}(p, q) \text { in } B^{\prime}, \quad q \text { fixed in } B^{\prime} .
$$

This function is discontinuous along $b^{\prime}$ but has continuous normal derivatives there. For this reason, we have

and hence

$$
D\left\{n(p, q), n^{*}(p, s)-n(p, s)\right\}
$$

$$
=\iint_{b} n(p, q) \frac{\partial}{\partial \nu}\left(\frac{1}{4 \pi r(p, s)}+n(p, s)\right) d \sigma=0
$$




$$
\begin{aligned}
D\left\{n^{*}(p, q), n^{*}(p, s)\right\}= & D\{n(p, q), n(p, s)\} \\
& +D\left\{n^{*}(p, q)-n(p, q), n^{*}(p, s)-n(p, s)\right\} .
\end{aligned}
$$

As before, we transform this identity into the final form:

$$
\begin{aligned}
N^{\prime}(q, s)= & N(q, s)+\iiint_{\Sigma} \operatorname{grad} N(p, q) \cdot \operatorname{grad} N(p, s) d \tau \\
& +D^{\prime}\left\{N^{\prime}(p, q)-N(p, q), N^{\prime}(p, s)-N(p, s)\right\} .
\end{aligned}
$$

For the quadratic form

$$
A=\sum_{i, k=1}^{m} n\left(q_{i}, q_{k}\right) t_{i} t_{k}
$$

we obtain the identity

$$
\begin{aligned}
A^{\prime}=A & +\iiint_{\Sigma}\left|\operatorname{grad} \sum_{i=1}^{m} t_{i} N\left(p, q_{i}\right)\right|^{2} d \tau \\
& +D^{\prime}\left\{\sum_{i=1}^{m} t_{i}\left(N^{\prime}\left(p, q_{i}\right)-N\left(p, q_{i}\right)\right)\right\}
\end{aligned}
$$

whence the inequality

$$
A^{\prime} \geqq A+\iiint_{\Sigma}\left|\operatorname{grad} \sum_{i=1}^{m} t_{i} N\left(p, q_{i}\right)\right|^{2} d \tau
$$

This yields the following theorem: The quadratic form $\sum_{i, k=1}^{m} n\left(q_{i}, q_{k}\right) t_{i} t_{k}$ increases monotonically for fixed $q_{i}$ and $t_{i}$ if the surface $b$ expands.

We assume now that a correspondence $p \leftrightarrow p^{\prime}$ between points on $b$ and $b^{\prime}$ has been established such that the distance $\overline{p p^{\prime}}$ is less than $\epsilon$ and that, at the same time, the angle between the corresponding normals is less than $\epsilon$. In this case, we have again an asymptotic formula

$$
N^{\prime}(p, q)-N(p, q)=O(\epsilon)
$$

for $p, q \in B^{\prime}$

and the same is true for the partial derivatives of this difference. Thus, we are led to the following variational formula, which is an immediate consequence of (4):

$$
\delta N(q, s)=\delta n(q, s)=\iint_{b} \operatorname{grad} N(p, q) \cdot \operatorname{grad} N(p, s) \delta \nu d \sigma+O\left(\epsilon^{2}\right) .
$$

This formula, too, is due to Hadamard. (Cf. [5, p. 312].) We point out again that (4) may be used in order to determine the variation $\delta n(q, s)$ of the Neumann function in a higher degree of precision with respect to $\epsilon$.

(b) We apply next the same reasoning to the identity 


$$
\begin{aligned}
D\left\{n^{*}(p, q), g^{*}(p, s)\right\}= & D\{n(p, q), g(p, s)\} \\
& +D\left\{n^{*}(p, q)-n(p, q), g^{*}(p, s)-g(p, s)\right\} .
\end{aligned}
$$

We obtain after the usual transformations

$$
\begin{aligned}
0= & -\iiint_{\Sigma} \operatorname{grad} N(p, q) \cdot \operatorname{grad} G(p, s) d \tau \\
& +D^{\prime}\left\{N^{\prime}(p, q)-N(p, q), G^{\prime}(p, s)-G(p, s)\right\} .
\end{aligned}
$$

Applying Schwarz's inequality to (11) and using the identities 4.1 (10) and (6), we find easily the inequality:

$$
\begin{aligned}
& \left(\iiint_{\Sigma} \operatorname{grad} \sum_{i=1}^{m} t_{i} N\left(p, q_{i}\right) \cdot \operatorname{grad} \sum_{i=1}^{m} t_{i} G\left(p, q_{i}\right) d \tau\right)^{2} \\
& \leqq\left(Q^{\prime}-Q-\iiint_{\Sigma}\left|\operatorname{grad} \sum_{i=1}^{m} t_{i} G\left(p, q_{i}\right)\right|^{2} d \tau\right) \\
& \cdot\left(A^{\prime}-A-\iiint_{\Sigma}\left|\operatorname{grad} \sum_{i=1}^{m} t_{i} N\left(p, q_{i}\right)\right|^{2} d \tau\right) .
\end{aligned}
$$

Here we have an estimate for the simultaneous approximation of $Q^{\prime}$ and $A^{\prime}$ in the inequalities 4.1 (11) and (7). The left-hand side of the inequality contains only terms related to the original surface $b$ and is, therefore, to be considered as known. It should be remarked that on $b$ the gradients of $N$ and $G$ are orthogonal to each other so that the left-hand term in (12) is of order $\epsilon^{4}$ at least if $b$ and $b^{\prime}$ are in an $\epsilon$-neighborhood.

It may easily be seen that the inequality 2.3 (17) may be derived from a variational formula of type (12) if the exterior surface $b^{\prime}$ is kept fixed while $b$ is contracted into a point.

Finally, we obtain, in view of 4.1 (8) and (4), (11), the following formula for the change of the kernel function:

$$
\begin{aligned}
k^{\prime}(q, s)= & k(q, s)+\iiint_{\Sigma} \operatorname{grad} k(p, q) \cdot \operatorname{grad} k(p, s) d \tau \\
& +D^{\prime}\left\{k^{\prime}(p, q)-k(p, q), k^{\prime}(p, s)-k(p, s)\right\} .
\end{aligned}
$$

We see that the three fundamental functions $k(q, s), n(q, s)$, and $g(q, s)$ have exactly the same law of variation. We may conclude again that the quadratic form connected with $k(q, s)$ increases monotonically with $b$, but since $k(q, s)=n(q, s)+g(q, s)$ this is an obvious consequence of the corresponding theorems for $n$ and $g$. Also we may obtain for $k(q, s)$ a variational formula of the Hadamard type just in the same way as for Green's and Neumann's functions.

4.3. Conductor potential and capacity. In order to apply our method to 
the conductor potential $u(p)$ we start with the identity

$$
D\{g(p, q), u(p)\}=u(q)
$$

which follows immediately from 2.4 (1) and 2.4 (13). We define the function

$$
u^{*}(p)=1 \text { in } \Sigma, \quad u^{*}(p)=u^{\prime}(p) \text { in } B^{\prime}
$$

which is continuous on $b^{\prime}$. Since we have

(3) $D\left\{g(p, q), u^{*}(p)-u(p)\right\}=0, \quad D\left\{g^{*}(p, q)-g(p, q), u(p)\right\}=0$

we may write

$$
\begin{aligned}
D\left\{g^{*}(p, q), u^{*}(p)\right\}= & D\{g(p, q), u(p)\} \\
& +D\left\{g^{*}(p, q)-g(p, q), u^{*}(p)-u(p)\right\} .
\end{aligned}
$$

Using now the identity (1), we obtain for $q \in B^{\prime}$

$$
\begin{aligned}
u^{\prime}(q)= & u(q)-\iiint_{\Sigma} \operatorname{grad} G(p, q) \cdot \operatorname{grad} u(p) d \tau \\
& +D^{\prime}\left\{G^{\prime}(p, q)-G(p, q), u^{\prime}(p)-u(p)\right\} .
\end{aligned}
$$

By the definition of the capacity $C$ and 2.4 (13)

$$
\lim _{q \rightarrow \infty}\{r u(q)\}=C, \quad \lim _{q \rightarrow \infty}\{r g(p, q)\}=\frac{u(p)}{4 \pi}
$$

where $r$ is the distance of $q$ from the origin; hence

$$
C^{\prime}=C+\frac{1}{4 \pi} \iiint_{\Sigma}(\operatorname{grad} u)^{2} d \tau+\frac{1}{4 \pi} D^{\prime}\left(u^{\prime}-u\right)
$$

follows. This leads to the inequality

$$
C^{\prime} \geqq C+\frac{1}{4 \pi} \iiint_{\Sigma}|\operatorname{grad} u|^{2} d \tau .
$$

We obtain also the well known variational formula for the capacity (Poincaré $[13$, p. 20])

$$
\delta C=\frac{1}{4 \pi} \iint_{b}\left(\frac{\partial u}{\partial \nu}\right)^{2} \delta \nu d \sigma+O\left(\epsilon^{2}\right) .
$$

By means of the identity

$$
D\{n(p, q), u(p)\}=\frac{1}{4 \pi} \iint_{b} \frac{\partial}{\partial \nu}\left(\frac{1}{r(p, q)}\right) d \sigma=0
$$

we derive from 


$$
\begin{aligned}
D\left\{n^{*}(p, q), u^{*}(p)\right\}= & D\{n(p, q), u(p)\} \\
& +D\left\{n^{*}(p, q)-n(p, q), u^{*}(p)-u(p)\right\}
\end{aligned}
$$

the identity

$$
\begin{aligned}
0= & \iiint_{\Sigma} \operatorname{grad} N(p, q) \cdot \operatorname{grad} u(p) d \tau \\
& +D^{\prime}\left\{N^{\prime}(p, q)-N(p, q), u^{\prime}(p)-u(p)\right\} .
\end{aligned}
$$

Hence, using 4.2 (4) and (7) we obtain by means of Schwarz's inequality

$$
\begin{aligned}
\left(\iiint_{\Sigma} \operatorname{grad} N(p, q)\right. & \cdot \operatorname{grad} u(p) d \tau)^{2} \\
\leqq & \left(n^{\prime}(q, q)-n(q, q)-\iiint_{\Sigma}|\operatorname{grad} N(p, q)|^{2} d \tau\right) \\
& \cdot\left(4 \pi\left(C^{\prime}-C\right)-\iiint_{\Sigma}|\operatorname{grad} u|^{2} d \tau\right) .
\end{aligned}
$$

This is again a formula where the increments of two functionals are interrelated. It is obvious that numerous inequalities of this type may be obtained in the same way.

Another source of inequalities and estimates consists in combining various quantities in such a way that the variation of the composite quantity is positive; this shows that the combination is monotonic. Consider, for example,

$$
F=g(q, q)+2 \alpha u(q)+4 \pi C \alpha^{2} .
$$

In view of $4.1(8),(5)$, and (7), we have

$$
\begin{aligned}
F^{\prime}=F & +\iiint_{\Sigma}|\operatorname{grad}(G(p, q)-\alpha u(p))|^{2} d \tau \\
& +D^{\prime}\left(G^{\prime}(p, q)-G(p, q)+\alpha\left(u^{\prime}-u\right)\right) .
\end{aligned}
$$

This leads to the inequality

$$
F^{\prime} \geqq F+\iiint_{\Sigma}|\operatorname{grad}(G(p, q)-\alpha u(p))|^{2} d \tau
$$

and shows that $F$ increases monotonously with expanding $b$. Many other combinations of this kind are possible.

4.4. Variational formulas for the $\psi_{i}$ and $\phi_{i}$. We deal finally with variational formulas for the functions $\psi_{i}$ and $\phi_{i}$. These might be derived immediately from the corresponding formulas for Green's and Neumann's functions in view of the fact that the $\psi_{i}$ and $\phi_{i}$ are certain coefficients in the expansion 
of these functions near infinity. It is, however, easy to derive these formulas directly in the same way which was followed in the previous sections.

We define in $B$ the two triplets of functions

$$
\begin{array}{llll}
\psi_{i}^{*}=x_{i}-\alpha_{i}^{\prime} \text { in } \Sigma, & \psi_{i}^{*}=\psi_{i}^{\prime} \text { in } B^{\prime}, & i=1,2,3, \\
\phi_{i}{ }^{*}=-x_{i} & \text { in } \Sigma, & \phi_{i}^{*}=\phi_{i}^{\prime} \text { in } B^{\prime}, & i=1,2,3 .
\end{array}
$$

The first three functions are continuous on $b^{\prime}$ but have discontinuous derivatives. The second triplet of functions is discontinuous on $b^{\prime}$ but possesses continuous normal derivatives through $b^{\prime}$.

Using the continuity of the $\psi_{i}^{*}$, we have the identity

$$
\begin{aligned}
D\left\{g^{*}(p, q), \psi_{i}^{*}(p)\right\}= & D\left\{g(p, q), \psi_{i}(p)\right\}+D\left\{g(p, q), \psi_{i}^{*}-\psi_{i}\right\} \\
& +D\left\{g^{*}(p, q)-g(p, q), \psi_{i}^{*}(p)-\psi_{i}(p)\right\} .
\end{aligned}
$$

Now obviously

$$
\begin{aligned}
& D\left\{g(p, q), \psi_{i}^{*}(p)-\psi_{i}(p)\right\} \\
& \quad=\left(\alpha_{i}^{\prime}-\alpha_{i}\right) \iint_{b} \frac{\partial}{\partial \nu} g(p, q) d \sigma=-\left(\alpha_{i}^{\prime}-\alpha_{i}\right) u(q) .
\end{aligned}
$$

Thus, we obtain from (3) by using the identity 2.9 (1) and the corresponding result with respect to $b^{\prime}$ :

$$
\begin{aligned}
\psi_{i}^{\prime}(q)= & \psi_{i}(q)-\left(\alpha_{i}^{\prime}-\alpha_{i}\right) u(q)+\iiint_{\Sigma} \operatorname{grad} G(p, q) \cdot \operatorname{grad} \Psi_{i}(p) d \tau \\
& +D^{\prime}\left\{g^{\prime}(p, q)-g(p, q), \psi_{i}^{\prime}(p)-\psi_{i}(p)\right\} .
\end{aligned}
$$

Since $\alpha_{i}$ has been identified in 2.7 (16) with a combination of coefficients of $u$, we have a complete variation formula for $\psi_{i}(q)$.

Combining now $\psi_{i}$ with Neumann's function and using the identity

$$
\begin{aligned}
D\left\{n^{*}(p, q), \psi_{i}^{*}(p)\right\}= & D\left\{n(p, q), \psi_{i}(p)\right\} \\
& +D\left\{n^{*}(p, q)-n(p, q), \psi_{i}^{*}(p)-\psi_{i}(p)\right\}
\end{aligned}
$$

we obtain by means of 2.9 (3):

$$
\begin{aligned}
0= & -\iiint_{\Sigma} \operatorname{grad} N(p, q) \cdot \operatorname{grad} \Psi_{i}(p) d \tau \\
& +D^{\prime}\left\{n^{\prime}(p, q)-n(p, q), \psi_{i}^{\prime}(p)-\psi_{i}(p)\right\} .
\end{aligned}
$$

Next, we start from the identity

$$
\begin{aligned}
D\left\{n^{*}(p, q), \phi_{i}^{*}(p)\right\}=D\{n(p, q), & \left.\phi_{i}(p)\right\} \\
& +D\left\{n^{*}(p, q)-n(p, q), \phi_{i}^{*}(p)-\phi_{i}(p)\right\} .
\end{aligned}
$$

Using 2.9 (5), we obtain 


$$
\begin{aligned}
\phi_{i}^{\prime}(q)= & \phi_{i}(q)+\iiint_{\Sigma} \operatorname{grad} N(p, q) \cdot \operatorname{grad} \Phi_{i}(p) d \tau \\
& +D^{\prime}\left\{n^{\prime}(p, q)-n(p, q), \phi_{i}^{\prime}(p)-\phi_{i}(p)\right\} .
\end{aligned}
$$

From the identity

$$
\begin{aligned}
D\left\{g^{*}(p, q), \phi_{i}^{*}(p)\right\}= & D\left\{g(p, q), \phi_{i}(p)\right\} \\
& +D\left\{g^{*}(p, q)-g(p, q), \phi_{i}^{*}(p)-i(p)\right\}
\end{aligned}
$$

we obtain by virtue of the identity 2.9 (4):

$$
\begin{aligned}
0= & -\iiint_{\Sigma} \operatorname{grad} G(p, q) \cdot \operatorname{grad} \Phi_{i}(p) d \tau \\
& +D^{\prime}\left\{g^{\prime}(p, q)-g(p, q), \phi_{i}^{\prime}(p)-\phi_{i}(p)\right\} .
\end{aligned}
$$

Finally, we may combine $\psi_{i}$ and $\phi_{k}$. We start with the identities

$$
D\left(\psi_{i}^{*}, \psi_{k}^{*}\right)=D\left(\psi_{i}, \psi_{k}\right)+D\left\{\psi_{i}^{*}-\psi_{i}, \psi_{k}^{*}-\psi_{k}\right\}
$$

and obtain in view of 1.5 (16)

(13) $4 \pi e_{i k}^{\prime}=4 \pi e_{i k}+\iiint_{\Sigma} \operatorname{grad} \Psi_{i} \cdot \operatorname{grad} \Psi_{k} d \tau+D^{\prime}\left\{\psi_{i}^{\prime}-\psi_{i}, \psi_{k}^{\prime}-\psi_{k}\right\}$.

Similarly, starting with

$$
D\left(\phi_{i}^{*}, \phi_{k}^{*}\right)=D\left(\phi_{i}, \phi_{k}\right)+D\left\{\phi_{i}{ }^{*}-\phi_{i}, \phi_{k}{ }^{*}-\phi_{k}\right\}
$$

we arrive at

(15) $4 \pi d_{i k}^{\prime}=4 \pi d_{i k}+\iiint_{\Sigma} \operatorname{grad} \Phi_{i} \cdot \operatorname{grad} \Phi_{k} d \tau+D^{\prime}\left\{\phi_{i}^{\prime}-\phi_{i}, \phi_{k}^{\prime}-\phi_{k}\right\}$.

Finally, using the identity

$$
D\left(\psi_{i}^{*}, \phi_{k}^{*}\right)=D\left(\psi_{i}, \phi_{k}\right)+D\left\{\psi_{i}^{*}-\psi_{i}, \phi_{k}{ }^{*}-\phi_{k}\right\}
$$

we obtain in view of 1.5 (18)

$$
0=-\iiint_{\Sigma} \operatorname{grad} \Psi_{i} \cdot \operatorname{grad} \Phi_{k} d \tau+D^{\prime}\left\{\psi_{i}^{\prime}-\psi_{i}, \phi_{k}^{\prime}-\phi_{k}\right\} .
$$

From the formulas (13), (15), and (17) we draw the following conclusions: The dipole form $\sum_{i, k=1,2,3} e_{i k} h_{i} h_{k}$ of the polarization is for fixed $h_{i}$ a monotonically increasing function of $b$ and satisfies the inequality

$$
\sum_{i, k=1,2,3} e_{i k}^{\prime} h_{i} h_{k} \geqq \sum_{i, k=1,2,3} e_{i k} h_{i} h_{k}+\frac{1}{4 \pi} \iiint_{\Sigma}\left|\operatorname{grad} \sum_{i=1}^{3} h_{i} \Psi_{i}\right|^{2} d \tau .
$$

For the first part of this assertion cf. 3.8. Assuming an infinitesimal variation 
of $b$, we have the formula of Hadamard type:

$$
\delta e_{i k}=\frac{1}{4 \pi} \iint_{b} \frac{\partial \Psi_{i}}{\partial \nu} \frac{\partial \Psi_{k}}{\partial \nu} \delta \nu d \sigma+O\left(\epsilon^{2}\right) .
$$

Similarly we conclude in the virtual mass problem: The dipole form $\sum_{i, k=1,2,3} d_{i k} h_{i} h_{k}$ of the virtual mass is for fixed $h_{i}$ a monotonically increasing function of $b$ and satisfies the inequality

$$
\sum_{i, k=1,2,3} d_{i k}^{\prime} h_{i} h_{k} \geqq \sum_{i, k=1,2,3} d_{i k} h_{i} h_{k}+\frac{1}{4 \pi} \iiint_{\Sigma}\left|\operatorname{grad} \sum_{i=1}^{3} h_{i} \Phi_{i}\right|^{2} d \tau .
$$

(Cf. 3.8.) We have the variational formula:

$$
\delta d_{i k}=\frac{1}{4 \pi} \iint_{b} \operatorname{grad} \Phi_{i} \cdot \operatorname{grad} \Phi_{k} \delta \nu d \sigma+O\left(\epsilon^{2}\right) .
$$

Combining finally (13), (15), and (17) by means of Schwarz's inequality, we find

$$
\begin{aligned}
\left(\iiint_{\Sigma} \operatorname{grad}\right. & \left.\sum_{i=1}^{3} h_{i} \Psi_{i} \cdot \operatorname{grad} \sum_{i=1}^{3} h_{i}^{\prime} \Phi_{i} d \tau\right)^{2} \\
\leqq & \left(\sum_{i, k=1,2,3}\left(e_{i k}^{\prime}-e_{i k}\right) h_{i} h_{k}-\frac{1}{4 \pi} \iiint_{\Sigma}\left|\operatorname{grad} \sum_{i=1}^{3} h_{i} \Psi_{i}\right|^{2} d \tau\right) . \\
& \cdot\left(\sum_{i, k=1,2,3}\left(d_{i k}^{\prime}-d_{i k}\right) h_{i}^{\prime} h_{k}^{\prime}-\frac{1}{4 \pi} \iiint_{\Sigma}\left|\operatorname{grad} \sum_{i=1}^{3} h_{i}^{\prime} \Phi_{i}\right|^{2} d \tau\right) .
\end{aligned}
$$

We mention at last the formula

$$
D\left(u^{*}, \psi_{i}^{*}\right)=D\left(u, \psi_{i}\right)+D\left(u, \psi_{i}^{*}-\psi_{i}\right)+D\left\{u^{*}-u, \psi_{i}^{*}-\psi_{i}\right\}
$$

Since $D\left(u, \psi_{i}\right)=0$ and

$$
D\left\{u, \psi_{i}^{*}-\psi_{i}\right\}=\left(\alpha_{i}^{\prime}-\alpha_{i}\right) \iint_{b} \frac{\partial u}{\partial \nu} d \sigma=-4 \pi C\left(\alpha_{i}^{\prime}-\alpha_{i}\right)
$$

we derive from (23):

$$
\begin{aligned}
0=-4 \pi C\left(\alpha_{i}^{\prime}-\alpha_{i}\right) & -\iiint_{\Sigma} \operatorname{grad} u \cdot \operatorname{grad} \Psi_{i} d \tau \\
& +D^{\prime}\left\{u^{\prime}-u, \psi_{i}^{\prime}-\psi_{i}\right\} .
\end{aligned}
$$

By means of this formula the difference $\alpha_{i}^{\prime}-\alpha_{i}$ can be eliminated from (5). Formula (25) might also have been obtained directly from (5) by comparing there on both sides the terms of order $r^{-1}$ at infinity.

4.5. Polarization of nearly spherical solids. We consider now nearly spherical domains. The variational formulas announced in 1.11 involve terms up 
to the second order. The first order terms in 1.11 (3) and (4) follow easily from 4.4 (19) and (21).

Dealing with the formula 1.11 (4) for the average polarization of a nearly spherical solid we follow an analogous procedure as in the case of the average virtual mass. (Cf. Szegö [19].) We follow also the notation of that paper [19] to which we shall refer repeatedly. It is advisable to compute first the polarization $P$ in an arbitrary direction $h$.

(a) We introduce the vector function $\psi$ defined by the condition

$$
\psi=\boldsymbol{r}+\text { const. }
$$

on the boundary surface of the given solid, and having the usual dipole behavior at infinity. We write

$$
\psi=t \cdot r^{-2}+u+v
$$

where $r=r t$; assuming that $r=1+f=1+\epsilon \bar{f}$ where $\bar{f}$ is fixed, the harmonic functions $u$ and $v$ will be of the first and second order, respectively, as $\epsilon \rightarrow 0$.

Inserting (2) into (1) we obtain

$$
\boldsymbol{t}\left(1-2 f+3 f^{2}\right)+u+f \frac{\partial u}{\partial r}+v=(1+f) t+\text { const. }
$$

so that

$$
\begin{aligned}
& u=3 f t+\text { const., } \\
& v=-3 f^{2} t-f \partial u / \partial r+\text { const. }
\end{aligned}
$$

The derivatives with respect to $r$ are taken for $r=1$.

(b) In order to compute $P$ we need $[19, \S 2,(8)]$

$$
\begin{aligned}
\frac{\partial(\boldsymbol{h} \cdot \boldsymbol{\psi})}{\partial \nu}= & \frac{\partial(\boldsymbol{h} \cdot \boldsymbol{\psi})}{\partial r}-\frac{1}{r} \nabla(f, \boldsymbol{h} \cdot \boldsymbol{\psi})+\frac{f}{r} \nabla(f, \boldsymbol{h} \cdot \boldsymbol{\psi})-\frac{1}{2} \nabla(f) \frac{\partial(\boldsymbol{h} \cdot \boldsymbol{\psi})}{\partial r} \\
= & -\frac{2}{r^{3}} \boldsymbol{h} \cdot \boldsymbol{t}+\frac{\partial}{\partial r}(\boldsymbol{h} \cdot \boldsymbol{u})+\frac{\partial}{\partial r}(\boldsymbol{h} \cdot \boldsymbol{v})-\frac{1}{r^{3}} \nabla(f, \boldsymbol{h} \cdot \boldsymbol{t}) \\
& -\frac{1}{r} \nabla(f, \boldsymbol{h} \cdot \boldsymbol{u})+f \nabla(f, \boldsymbol{h} \cdot \boldsymbol{t})+\nabla(f) \boldsymbol{h} \cdot \boldsymbol{t}
\end{aligned}
$$

so that

$$
\left(\frac{\partial}{\partial \nu}(\boldsymbol{h} \cdot \psi)\right)_{r=1}=-2 \boldsymbol{h} \cdot \boldsymbol{t}\left(1-3 f+6 f^{2}\right)+\frac{\partial}{\partial r}(\boldsymbol{h} \cdot \boldsymbol{u})+f \frac{\partial^{2}}{\partial r^{2}}(\boldsymbol{h} \cdot \boldsymbol{u})
$$

$$
\begin{aligned}
& +\frac{\partial}{\partial \boldsymbol{r}}(\boldsymbol{h} \cdot \boldsymbol{v})-\nabla(f, \boldsymbol{h} \cdot \boldsymbol{t})+4 f \nabla(f, \boldsymbol{h} \cdot \boldsymbol{t}) \\
& -\nabla(f, \boldsymbol{h} \cdot \boldsymbol{u})+\nabla(f) \boldsymbol{h} \cdot \boldsymbol{t} .
\end{aligned}
$$


Here and later the derivatives with respect to $r$ are taken at $r=1$.

(c) Now $[19, \S 4(2)]$

$$
\boldsymbol{h} \cdot \boldsymbol{r} d \sigma=\boldsymbol{h} \cdot \boldsymbol{t}\left(1+3 f+3 f^{2}+\frac{1}{2} \nabla(f)\right) d \boldsymbol{\omega}
$$

so that

$$
\begin{aligned}
&-(\boldsymbol{h} \cdot \boldsymbol{r}) \frac{\partial}{\partial \nu}(\boldsymbol{h} \cdot \boldsymbol{\psi}) d \sigma \\
&=\left\{2(\boldsymbol{h} \cdot \boldsymbol{t})^{2}-(\boldsymbol{h} \cdot \boldsymbol{t}) \frac{\partial}{\partial r}(\boldsymbol{h} \cdot \boldsymbol{u})+(\boldsymbol{h} \cdot \boldsymbol{t}) \nabla(f, \boldsymbol{h} \cdot \boldsymbol{t})\right\} d \boldsymbol{\omega} \\
&+(\boldsymbol{h} \cdot \boldsymbol{t})\left\{-f \frac{\partial^{2}}{\partial r^{2}}(\boldsymbol{h} \cdot \boldsymbol{u})-3 f \frac{\partial}{\partial r}(\boldsymbol{h} \cdot \boldsymbol{u})\right. \\
&\left.-\frac{\partial}{\partial r}(\boldsymbol{h} \cdot \boldsymbol{v})-f \nabla(f, \boldsymbol{h} \cdot \boldsymbol{t})+\nabla(f, \boldsymbol{h} \cdot \boldsymbol{u})\right\} d \omega .
\end{aligned}
$$

Hence we obtain for the polarization

$$
P=\frac{8 \pi}{3}+P^{\prime}+P^{\prime \prime}
$$

where $P^{\prime}$ and $P^{\prime \prime}$ denote terms of the first and second order, respectively.

In view of the orthogonality of the surface harmonics we can replace

$$
-(h \cdot t) \frac{\partial}{\partial r}(h \cdot u) \text { and }-(h \cdot t) \frac{\partial}{\partial r}(h \cdot v)
$$

by $2(\boldsymbol{h} \cdot \boldsymbol{t})(\boldsymbol{h} \cdot \boldsymbol{u})$ and $2(\boldsymbol{h} \cdot \boldsymbol{t})(\boldsymbol{h} \cdot \boldsymbol{v})$, respectively. Consequently $[19, \S 4,(2)]$

$$
\begin{aligned}
P^{\prime} & =\iint\left\{2(\boldsymbol{h} \cdot \boldsymbol{t})(\boldsymbol{h} \cdot \boldsymbol{u})+\frac{1}{2} \nabla\left(f,(\boldsymbol{h} \cdot \boldsymbol{t})^{2}\right)\right\} d \omega \\
& =\iint\left\{6 f(\boldsymbol{h} \cdot \boldsymbol{t})^{2}-\frac{1}{2} f \Delta(\boldsymbol{h} \cdot \boldsymbol{t})^{2}\right\} d \omega=\iint\left\{9 f(\boldsymbol{h} \cdot \boldsymbol{t})^{2}-f\right\} d \omega \\
& =2 \iint f d \omega+6 \iint f P_{2}(\boldsymbol{h} \cdot \boldsymbol{t}) d \omega=8 \pi X_{0}+\frac{24 \pi}{5} X_{2},
\end{aligned}
$$

where $X_{2}$ has to be taken at the end point of $h$.

(d) For $r=1$ we have $[19, \S 2(9)]$

$$
-\frac{\partial^{2}}{\partial r^{2}}(\boldsymbol{h} \cdot \boldsymbol{u})=2 \frac{\partial}{\partial r}(\boldsymbol{h} \cdot \boldsymbol{u})+\Delta(\boldsymbol{h} \cdot \boldsymbol{u})=2 \frac{\partial}{\partial r}(\boldsymbol{h} \cdot \boldsymbol{u})+3 \Delta(f \boldsymbol{h} \cdot \boldsymbol{t})
$$

so that 


$$
\begin{aligned}
P^{\prime \prime}= & \iint\left\{-f(\boldsymbol{h} \cdot \boldsymbol{t}) \frac{\partial}{\partial r}(\boldsymbol{h} \cdot \boldsymbol{u})+3 f(\boldsymbol{h} \cdot \boldsymbol{t}) \Delta(f \boldsymbol{h} \cdot \boldsymbol{t})\right. \\
& +2(\boldsymbol{h} \cdot \boldsymbol{t})\left(-3 f^{2}(\boldsymbol{h} \cdot \boldsymbol{t})-f \frac{\partial}{\partial r}(\boldsymbol{h} \cdot \boldsymbol{u})\right)-f(\boldsymbol{h} \cdot \boldsymbol{t}) \nabla(f, \boldsymbol{h} \cdot \boldsymbol{t}) \\
& +3(\boldsymbol{h} \cdot \boldsymbol{t}) \nabla(f, f \boldsymbol{h} \cdot \boldsymbol{t})\} d \omega .
\end{aligned}
$$

Now

$$
\begin{aligned}
-3 & \nabla(f \boldsymbol{h} \cdot \boldsymbol{t})-f(\boldsymbol{h} \cdot \boldsymbol{t}) \nabla(f, \boldsymbol{h} \cdot \boldsymbol{t})+3(\boldsymbol{h} \cdot \boldsymbol{t}) \nabla(f, f \boldsymbol{h} \cdot \boldsymbol{t}) \\
= & -3 f^{2} \nabla(\boldsymbol{h} \cdot \boldsymbol{t})-3(\boldsymbol{h} \cdot \boldsymbol{t})^{2} \nabla(f)-6 f(\boldsymbol{h} \cdot \boldsymbol{t}) \nabla(f, \boldsymbol{h} \cdot \boldsymbol{t})-f(\boldsymbol{h} \cdot \boldsymbol{t}) \nabla(f, \boldsymbol{h} \cdot \boldsymbol{t}) \\
& \quad+3 f(\boldsymbol{h} \cdot \boldsymbol{t}) \nabla(f, \boldsymbol{h} \cdot \boldsymbol{t})+3(\boldsymbol{h} \cdot \boldsymbol{t})^{2} \nabla(f) \\
= & -3 f^{2}\left(1-(\boldsymbol{h} \cdot \boldsymbol{t})^{2}\right)-4 f(\boldsymbol{h} \cdot \boldsymbol{t}) \nabla(f, \boldsymbol{h} \cdot \boldsymbol{t}) \\
= & -3 f^{2}\left(1-(\boldsymbol{h} \cdot \boldsymbol{t})^{2}\right)-\nabla\left(f^{2},(\boldsymbol{h} \cdot \boldsymbol{t})^{2}\right) .
\end{aligned}
$$

Hence

$$
\begin{aligned}
P^{\prime \prime}= & \iint\left\{-(\boldsymbol{h} \cdot \boldsymbol{u}) \frac{\partial}{\partial r}(\boldsymbol{h} \cdot \boldsymbol{u})-6 f^{2}(\boldsymbol{h} \cdot \boldsymbol{t})^{2}-3 f^{2}\left(1-(\boldsymbol{h} \cdot \boldsymbol{t})^{2}\right)\right. \\
& \left.+f^{2}\left(2-6(\boldsymbol{h} \cdot \boldsymbol{t})^{2}\right)\right\} d \omega \\
= & \iint\left\{-(\boldsymbol{h} \cdot \boldsymbol{u}) \frac{\partial}{\partial \boldsymbol{r}}(\boldsymbol{h} \cdot \boldsymbol{u})-9 f^{2}(\boldsymbol{h} \cdot \boldsymbol{t})^{2}-f^{2}\right\} d \omega .
\end{aligned}
$$

Recapitulating, we find for the polarization

(15) $P=\frac{8 \pi}{3}+8 \pi X_{0}+\frac{24 \pi}{5} X_{2}-\iint\left\{(\boldsymbol{h} \cdot \boldsymbol{u}) \frac{\partial}{\partial r}(\boldsymbol{h} \cdot \boldsymbol{u})+9 f^{2}(\boldsymbol{h} \cdot \boldsymbol{t})^{2}+f^{2}\right\} d \omega$

and, in particular, for the average polarization

$$
P_{m}=\frac{8 \pi}{3}+8 \pi X_{0}-\iint\left\{\frac{1}{3} \boldsymbol{u} \cdot \frac{\partial u}{\partial r}+4 f^{2}\right\} d \omega .
$$

4.6. Representation in terms of the variation. (a) Introducing the Laplace series 1.11 (2) for the variation $f$ of the unit sphere, we express now the average polarization $P_{m}$ in terms of the surface harmonics $X_{n}$ occurring in this series. Let $f$ and $g$ be two scalar functions defined on the unit sphere. As in $[19, \S 5]$, we introduce the symbol $[f, g]$ defined as follows. We determine the harmonic vector functions $u$ and $u_{1}$ by

$$
u=3 f t+\text { const., } \quad u_{1}=3 g t+\text { const. } \quad \text { for } r=1,
$$


assuming that $u$ and $u_{1}$ have the leading term const. $\cdot r^{-2}$ at infinity. Then

$$
[f, g]=-\iint u \frac{\partial u_{1}}{\partial \nu} d \omega=-\iint u_{1} \frac{\partial u}{\partial \nu} d \omega .
$$

Our aim is to prove that if $f$ has the Laplace series 1.11 (2),

$$
[f, f]=9 \sum_{n=0}^{\infty} \frac{2 n^{2}+3 n+2}{2 n+1} \iint X_{n}^{2} d \omega
$$

here $2 n^{2}+3 n+2$ has to be replaced by 6 for $n=1$.

(b) Let us consider the special case

$$
f(\theta, \phi)=P_{n}(\cos \theta) \text {. }
$$

Then $[19, \S 5,(2)]$

$$
\begin{aligned}
3 f t= & 3 \sin \theta P_{n}(\cos \theta)\left(i_{1} \cos \phi+i_{2} \sin \phi\right)+3 \cos \theta P_{n}\left(\cos _{-}^{\top} \theta\right) i_{3} \\
= & \frac{3}{2 n+1}\left\{\left(P_{n+1}^{\prime}(\cos \theta)-P_{n-1}^{\prime}(\cos \theta)\right) \sin \theta\left(i, \cos \phi+i_{2} \sin \phi\right)\right. \\
& \left.+\left((n+1) P_{n+1}(\cos \theta)+n P_{n-1}(\cos \theta)\right) i_{3}\right\},
\end{aligned}
$$

so that

$$
\begin{aligned}
u= & \frac{3}{2 n+1}\left\{\left(\frac{\sin \theta P_{n+1}^{\prime}(\cos \theta)}{r^{n+2}}-\frac{\sin \theta P_{n-1}^{\prime}(\cos \theta)}{r^{n}}\right)\left(i_{1} \cos \phi+i_{2} \sin \phi\right)\right. \\
& \left.+\left(\frac{(n+1) P_{n+1}(\cos \theta)}{r^{n+2}}+\frac{n P_{n-1}(\cos \theta)}{r^{n}}\right) i_{3}\right\} .
\end{aligned}
$$

For $n=0$ and $n=1$ the terms with $r^{-n}$ have to be replaced by 0 . Hence in view of the orthogonality of the six terms in (6):

$$
\begin{aligned}
{\left[P_{n}, P_{n}\right]=} & \frac{9}{(2 n+1)^{2}} \iint\left\{(n+2)\left(\sin \theta P_{n+1}^{\prime}(\cos \theta)\right)^{2}\right. \\
& +n\left(\sin \theta P_{n-1}^{\prime}(\cos \theta)\right)^{2}+(n+2)(n+1)^{2}\left(P_{n+1}(\cos \theta)\right)^{2} \\
& \left.+n^{3}\left(P_{n-1}(\cos \theta)\right)^{2}\right\} d \omega \\
= & \frac{18 \pi}{(2 n+1)^{2}}\left\{(n+2) \frac{2(n+1)(n+2)}{2 n+3}+n \frac{2(n-1) n}{2 n-1}\right. \\
& \left.+(n+2)(n+1)^{2} \frac{2}{2 n+3}+n^{3} \frac{2}{2 n-1}\right\} \\
= & \frac{36 \pi\left(2 n^{2}+3 n+2\right)}{(2 n+1)^{2}}=\frac{9\left(2 n^{2}+3 n+2\right)}{2 n+1} \iint\left(P_{n}(\cos \theta)\right)^{2} d \omega
\end{aligned}
$$


with an obvious modification for $n=1$.

The further steps leading to (3) are exactly the same as in the case of the virtual mass.

\section{Chapter V. Examples}

Most of our examples deal with the problem of polarization. We add very little to the problem of the virtual mass. The method used is the same in both cases; it is based on proper curvilinear coordinates and characteristic functions. But the resulting conditions are much simpler in the case of polarization. For the solids to be considered below, this problem can be settled for every choice of the unit vector $h$ whereas the problem of the virtual mass has been studied only in certain directions. An exception is the ellipsoid in which case complete results can be obtained.

Our notation follows that used in Szegö [18, pp. 343-349] with the only exception of the lens. The modified notation used in this case has been explained in 1.13 (e).

Throughout this chapter the symbol $\sum^{\prime}, n=0,1,2, \cdots$, has the meaning that the term $n=0$ of the summation has to be multiplied by $1 / 2$.

5.1. Virtual mass and polarization of the ellipsoid. In case of the ellipsoid we treat these two problems together proving the inequalities 1.12 (5) and 1.13 (3). The meaning of these inequalities is the following: Among all ellipsoids of given volume the sphere has the smallest average in virtual mass and polarization. (Cf. Pólya [14].) For the quantities 1.12 (3) we have, using the symbol $H=H(s)$ of $1.12(1)$ :

$$
\begin{aligned}
p_{1}+p_{2}+p_{3} & =2 \int_{0}^{\infty}\left(\frac{1}{a_{1}^{2}+s}+\frac{1}{a_{2}^{2}+s}+\frac{1}{a_{3}^{2}+s}\right) \frac{d s}{H} \\
& =4 \int_{0}^{\infty} \frac{H^{\prime} d s}{H^{2}}=4
\end{aligned}
$$

so that in viewlof the convexity of the function $x /(4-x)$ we conclude:

$$
\sum_{i=1}^{3} \frac{p_{i}}{4-p_{i}} \geqq 3 \frac{4 / 3}{4-4 / 3}=\frac{3}{2}
$$

which proves 1.12 (5). In order to prove inequality 1.13 (3) we use Cauchy's inequality:

$$
\sum_{i=1}^{3} \frac{1}{p_{i}} \geqq 9\left(\sum_{i=1}^{3} p_{i}\right)^{-1}=\frac{9}{4}
$$

so that

$$
\sum_{i=1}^{3} \frac{4-p_{i}}{p_{i}} \geqq 4 \frac{9}{4}-3=6
$$


The inequality $W P \geqq V^{2}$ takes in this case the form

$$
\sum_{i=1}^{3} \frac{p_{i}}{4-p_{i}} h_{i}^{2} \sum_{i=1}^{3} \frac{4-p_{i}}{p_{i}} h_{i}^{2} \geqq 1
$$

which follows from Cauchy's inequality.

5.2. Virtual mass of the anchor ring. Following Hicks [8] we write for the velocity potential in the direction of the axis (z-direction)

$$
\phi_{z}=(2 \operatorname{ch} \eta-2 \cos \theta)^{1 / 2} \sum_{n=1}^{\infty} A_{n} P_{n-1 / 2}(\operatorname{ch} \eta) \sin n \theta
$$

where the constants $A_{n}$ are determined from the boundary condition

$$
-\frac{\partial \phi_{z}}{\partial \eta}=\frac{\partial z}{\partial \eta}
$$

or

$$
\begin{aligned}
\sum_{n=1}^{\infty} A_{n} P_{n-1 / 2}\left(\operatorname{ch} \eta_{0}\right) \sin n \theta+\left(2 \operatorname{ch} \eta_{0}\right. & -2 \cos \theta) \sum_{n=1}^{\infty} A_{n} P_{n-1 / 2}^{\prime}\left(\operatorname{ch} \eta_{0}\right) \sin n \theta \\
& =4 a \sin \theta\left(2 \operatorname{ch} \eta_{0}-2 \cos \theta\right)^{-3 / 2}
\end{aligned}
$$

Here, $\eta, \theta$ are toroidal coordinates. We use the identity

$$
\frac{2}{\pi} \sum_{n=0}^{\infty} Q_{n-1 / 2}(\operatorname{ch} \eta) \cos n \theta=(2 \operatorname{ch} \eta-2 \cos \theta)^{-1 / 2}
$$

and another one arising from (4) by differentiation with respect to $\theta$. Moreover, we use the following identities $\left({ }^{7}\right)$ :

$$
\begin{aligned}
& P_{n-1 / 2}(z)+2 z P_{n-1 / 2}^{\prime}(z)=P_{n+1 / 2}^{\prime}(z)+P_{n-3 / 2}^{\prime}(z), \\
& 2 n Q_{n-1 / 2}(z)=Q_{n+1 / 2}^{\prime}(z)-Q_{n-3 / 2}^{\prime}(z), \\
& P_{n-1 / 2}^{\prime}(z) Q_{n+1 / 2}^{\prime}(z)-P_{n+1 / 2}^{\prime}(z) Q_{n-1 / 2}^{\prime}(z)=\frac{n+1 / 2}{z^{2}-1}, \quad z=\operatorname{ch} \eta .
\end{aligned}
$$

Thus we conclude from (3)

$$
\begin{aligned}
& -\left(A_{n-1}-A_{n}\right) P_{n-3 / 2}^{\prime}\left(\operatorname{ch} \eta_{0}\right)+\left(A_{n}-A_{n+1}\right) P_{n+1 / 2}^{\prime}\left(\operatorname{ch} \eta_{0}\right) \\
& =\frac{4 a}{\pi}\left(Q_{n+1 / 2}^{\prime}\left(\operatorname{ch} \eta_{0}\right)-Q_{n-3 / 2}^{\prime}\left(\operatorname{ch} \eta_{0}\right)\right), \quad n=1,2,3, \cdots ; A_{0}=0 .
\end{aligned}
$$

Multiplying (6) by $P_{n-1 / 2}^{\prime}\left(\mathrm{ch} \eta_{0}\right)$ and adding these equations, we find

(7) Cf. Whittaker-Watson, A course of modern analysis, American edition, 1943, pp. 308, 318; Hicks $[8$, p. $626,(\gamma)]$. The notation of Hicks is different from ours: our functions $P_{n-1 / 2}(\mathrm{ch} \eta), Q_{n-1 / 2}(\mathrm{ch} \eta)$ are denoted in Hicks [8] by $\pi^{-1} P_{n}(\eta), Q_{n}(\eta)$. 


$$
\begin{aligned}
\left(A_{n}-A_{n+1}\right) P_{n+1 / 2}^{\prime} P_{n-1 / 2}^{\prime} & +c\left(\eta_{0}\right) \\
& =\frac{4 a}{\pi} \sum_{\nu=1}^{n}\left(Q_{\nu+1 / 2}^{\prime}-Q_{\nu-3 / 2}^{\prime}\right) P_{\nu-1 / 2}^{\prime} \\
& =\frac{4 a}{\pi} \sum_{\nu=1}^{n}\left(P_{\nu+1 / 2}^{\prime} Q_{\nu-1 / 2}^{\prime}+\frac{\nu+1 / 2}{\operatorname{sh}^{2} \eta_{0}}-P_{\nu-1 / 2}^{\prime} Q_{\nu-3 / 2}^{\prime}\right) \\
& =\frac{4 a}{\pi} P_{n+1 / 2}^{\prime} Q_{n-1 / 2}^{\prime}+\frac{2 a}{\pi} \frac{(n+1)^{2}}{\operatorname{sh}^{2} \eta_{0}}+c_{1}\left(\eta_{0}\right)
\end{aligned}
$$

where the terms $c\left(\eta_{0}\right), c_{1}\left(\eta_{0}\right)$ are independent of $n$. Introducing the symbol $x_{n}$ of 1.12 (7) we have with an appropriate constant $\gamma$

$$
\begin{aligned}
A_{n}-A_{n+1} & =\frac{4 a}{\pi} x_{n}+\frac{2 a}{\pi} \frac{(n+1)^{2}}{n+1 / 2}\left(x_{n+1}-x_{n}\right)+\frac{2 a \gamma}{\pi} \frac{x_{n+1}-x_{n}}{n+1 / 2} \\
& =\frac{4 a}{\pi} \frac{\left((n+1)^{2}+\gamma\right) x_{n+1}-\left(n^{2}+\gamma\right) x_{n}}{2 n+1}, \quad n=0,1,2, \cdots .
\end{aligned}
$$

Hence

$$
-A_{n+1}=\frac{4 a}{\pi} \sum_{\nu=0}^{n} \frac{\left((\nu+1)^{2}+\gamma\right) x_{\nu+1}-\left(\nu^{2}+\gamma\right) x_{\nu}}{2 \nu+1} .
$$

The constant $\gamma$ is determined by the condition that $\lim _{n \rightarrow \infty} A_{n}=0$ so that

$$
\sum_{\nu=0}^{\infty} \frac{\left((\nu+1)^{2}+\gamma\right) x_{\nu+1}-\left(\nu^{2}+\gamma\right) x_{\nu}}{2 \nu+1}=0
$$

which can be written also in the simpler form

$$
\sum_{n=0}^{\infty} \frac{\left(n^{2}+\gamma\right) x_{n}}{4 n^{2}-1}=0
$$

The dipole coefficient $d_{s}$ follows easily from (1). Since for $\eta \rightarrow 0, \theta \rightarrow 0, r \rightarrow \infty$

$$
(2 \operatorname{ch} \eta-2 \cos \theta)^{1 / 2} \theta \sim 4 a^{2} \frac{z}{r^{3}}
$$

we have

$$
\begin{aligned}
d_{z} & =4 a^{2} \sum_{n=1}^{\infty} A_{n} n=4 a^{2} \sum_{n=0}^{\infty}\left(A_{n}-A_{n+1}\right) \frac{n(n+1)}{2} \\
& =\frac{8 a^{3}}{\pi} \sum_{n=0}^{\infty} \frac{n(n+1)}{2 n+1}\left\{\left((n+1)^{2}+\gamma\right) x_{n+1}-\left(n^{2}+\gamma\right) x_{n}\right\} .
\end{aligned}
$$

In view of $(10)$ we may replace $n(n+1)$ by $n(n+1)+1 / 4=(n+1 / 2)^{2}$ so that 


$$
d_{z}=\frac{2 a^{3}}{\pi} \sum_{n=0}^{\infty}(2 n+1)\left\{\left((n+1)^{2}+\gamma\right) x_{n+1}-\left(n^{2}+\gamma\right) x_{n}\right\}
$$

from which 1.12 (6) follows.

Using

$$
V=2 \pi^{2} a^{3} \operatorname{ch} \eta_{0}\left(\operatorname{sh} \eta_{0}\right)^{-8}
$$

we may compute the virtual mass $W_{33}=W_{z}$. The resulting expression is simpler than that given by Hicks [8, p. 648 (44)].

5.3. Polarization of the anchor ring. This problem can be dealt with in a similar fashion as the foregoing one for the virtual mass. We use again toroidal coordinates.

(a) In the direction of the axis of the ring (z-direction) we write for the electric potential

$$
\psi_{z}=(2 \operatorname{ch} \eta-2 \cos \theta)^{1 / 2} \sum_{n=1}^{\infty} A_{n} P_{n-1 / 2}(\operatorname{ch} \eta) \sin n \theta
$$

the boundary condition being as follows:

$$
\psi_{z}=z+\text { const. }=2 a \sin \theta(2 \operatorname{ch} \eta-2 \cos \theta)^{-1}+\text { const. } \quad \eta=\eta_{0} .
$$

Since each term of this equation is an odd function of $\theta$ the constant must be 0 and in view of the identity 5.2 (4) (which has to be differentiated with respect to $\theta$ )

$$
A_{n}=\frac{4 a}{\pi} n \frac{Q_{n-1 / 2}\left(\operatorname{ch} \eta_{0}\right)}{P_{n-1 / 2}\left(\operatorname{ch} \eta_{0}\right)}
$$

so that if we make use of $5.2(12)$

$$
e_{z}=4 a^{2} \sum_{n=1}^{\infty} n A_{n}
$$

This establishes 1.13 (4) and (5).

(b) In the direction perpendicular to the axis (for instance in the $x$-direction) we employ the "tesseral functions"

$$
(2 \operatorname{ch} \eta-2 \cos \theta)^{1 / 2} \operatorname{sh} \eta P_{n-1 / 2}^{\prime}(\operatorname{ch} \eta) \cos \phi \cos n \theta
$$

and write

$$
\psi_{x}=(2 \operatorname{ch} \eta-2 \cos \theta)^{1 / 2} \sum_{n=0}^{\infty} A_{n} \operatorname{sh} \eta P_{n-1 / 2}^{\prime}(\operatorname{ch} \eta) \cos \phi \cos n \theta .
$$

The boundary condition is

(7) $\quad \psi_{x}=x+$ const. $=2 a \operatorname{sh} \eta \cos \phi(2 \operatorname{ch} \eta-2 \cos \theta)^{-1}+$ const., $\quad \eta=\eta_{0}$. 
Since each term of this equation contains $\cos \phi$ the constant term must be 0 . Making use of the identity

$$
\frac{2}{\pi} \sum_{n=0}^{\infty} Q_{n-1 / 2}^{\prime}(\operatorname{ch} \eta) \cos n \theta=-(2 \operatorname{ch} \eta-2 \cos \theta)^{-3 / 2},
$$

arising from 5.2 (4) by differentiation, we obtain for the coefficients of (6)

$$
A_{n} P_{n-1 / 2}^{\prime}\left(\operatorname{ch} \eta_{0}\right)=-\frac{4 a}{\pi} Q_{n-1 / 2}^{\prime}\left(\operatorname{ch} \eta_{0}\right) \text {. }
$$

Since for $\eta \rightarrow 0, \theta \rightarrow 0, r \rightarrow \infty$

$$
(2 \operatorname{ch} \eta-2 \cos \theta)^{1 / 2} \operatorname{sh} \eta \cos \phi \sim 4 a^{2} \frac{x}{r^{3}}
$$

we find

$$
e_{x}=4 a^{2} \sum_{n=0}^{\infty} A_{n} P_{n-1 / 2}^{\prime}(1)=2 a^{2} \sum_{n=0}^{\infty} A_{n}\left(n^{2}-1 / 4\right)
$$

from which 1.13 (6) and (7) follow.

5.4. Polarization of the spindle. (a) In the direction of the axis of the spindle ( $x$-direction) we write, using the notation in $[18$, p. 345$]$,

$$
\psi_{x}=(2 \operatorname{ch} \eta-2 \cos \theta)^{1 / 2} \int_{-\infty}^{\infty} A(q) L_{q}(-\cos \theta) \sin q \eta d q
$$

with the boundary condition

$$
\psi_{x}=x+\text { const. }=2 a(2 \operatorname{ch} \eta-2 \cos \theta)^{-1} \operatorname{sh} \eta+\text { const., } \quad \theta=\theta_{0} .
$$

Since each term of this equation is an odd function of $\eta$ the constant term must be 0 . Consequently,

$$
\int_{-\infty}^{\infty} A(q) L_{q}\left(-\cos \theta_{0}\right) \sin q \eta d q=2 a\left(2 \operatorname{ch} \eta-2 \cos \theta_{0}\right)^{-3 / 2} \operatorname{sh} \eta .
$$

We use the identity arising from

$$
\frac{1}{2} \int_{-\infty}^{\infty} \frac{L_{q}(\cos \theta)}{\operatorname{ch} q \pi} \cos q \eta d q=(2 \operatorname{ch} \eta-2 \cos \theta)^{-1 / 2}
$$

by differentiation with respect to $\eta$. Thus

$$
A(q) L_{q}\left(-\cos \theta_{0}\right)=a \frac{q L_{q}\left(\cos \theta_{0}\right)}{\operatorname{ch} q \pi}
$$

follows. This furnishes 1.13 (9). In view of $L_{q}(-1)=1$ and 


$$
(2 \operatorname{ch} \eta-2 \cos \theta)^{1 / 2} \eta \sim 4 a^{2} \frac{x}{r^{3}}
$$$$
\text { for } \eta=0, \theta=0, r=\infty
$$

we obtain $1.13(10)$.

(b) In the direction perpendicular to the axis (for instance in the $y$-direction) we employ the "tesseral functions"

$$
(2 \operatorname{ch} \eta-2 \cos \theta)^{1 / 2} \sin \theta L_{q}^{\prime}(-\cos \theta) \cos q \eta \cos \phi
$$

and write

$$
\text { (8) } \quad \psi_{y}=(2 \operatorname{ch} \eta-2 \cos \theta)^{1 / 2} \int_{-\infty}^{\infty} A(q) \sin \theta L_{q}^{\prime}(-\cos \theta) \cos q \eta \cos \phi d q \text {. }
$$

The boundary condition is in this case

$$
\psi_{y}=y+\text { const. }=2 a(2 \operatorname{ch} \eta-2 \cos \theta)^{-1} \sin \theta \cos \phi+\text { const., } \quad \theta=\theta_{0} .
$$

Since each term contains $\cos \phi$ the constant term must be 0 . Consequently,

$$
\begin{aligned}
\int_{-\infty}^{\infty} A(q) L_{q}^{\prime}\left(-\cos \theta_{0}\right) \cos q \eta d q & =2 a\left(2 \operatorname{ch} \eta-2 \cos \theta_{0}\right)^{-3 / 2} \\
& =a \int_{-\infty}^{\infty} \frac{L_{q}^{\prime}\left(\cos \theta_{0}\right)}{\operatorname{ch} q \pi} \cos q \eta d q ;
\end{aligned}
$$

this last integral representation follows from (4) by differentiation. Hence

$$
A(q) L_{q}^{\prime}\left(-\cos \theta_{0}\right)=a \frac{L_{q}^{\prime}\left(\cos \theta_{0}\right)}{\operatorname{ch} q \pi} .
$$

In view of $L_{q}^{\prime}(-1)=1 / 2\left(q^{2}+1 / 4\right)$ and

$$
(2 \operatorname{ch} \eta-2 \cos \theta)^{1 / 2} \sin \theta \cos \phi \sim 4 a^{2} \frac{y}{r^{3}}, \quad \eta \rightarrow 0, \theta \rightarrow 0, r \rightarrow \infty
$$

we obtain 1.13 (11) and (12).

5.5. Polarization of two spheres. (a) In the direction of the central line $(x$-direction) we write

(1) $\psi_{x}=(2 \operatorname{ch} \eta-2 \cos \theta)^{1 / 2} \sum_{n=0}^{\infty}\left(A_{n} e^{N \eta}+B_{n} e^{-N \eta}\right) P_{n}(\cos \theta), \quad N=n+\frac{1}{2}$, with the boundary condition

(2) $\sum_{n=0}^{\infty}\left(A_{n} e^{N \eta}+B_{n} e^{-N \eta}\right) P_{n}(\cos \theta)=2 a \operatorname{sh} \eta(2 \operatorname{ch} \eta-2 \cos \theta)^{-3 / 2}$

$$
+p(2 \operatorname{ch} \eta-2 \cos \theta)^{-1 / 2}, \quad \text { for } \eta=\alpha \text { and } \eta=\beta
$$


where $p$ is a constant. Using the identities

$$
\begin{aligned}
(2 \operatorname{ch} \alpha-2 \cos \theta)^{-1 / 2} & =\sum_{n=0}^{\infty} e^{N \alpha} P_{n}(\cos \theta), \\
\operatorname{sh} \alpha(2 \operatorname{ch} \alpha-2 \cos \theta)^{-3 / 2} & =-\sum_{n=0}^{\infty} N e^{N \alpha} P_{n}(\cos \theta)
\end{aligned}
$$

and the corresponding identities with $\alpha$ replaced by $-\beta$ we obtain, by comparing the coefficients of $P_{n}(\cos \theta)$,

$$
\begin{aligned}
& A_{n}=\frac{(4 a N \operatorname{ch} N \alpha-2 p \operatorname{sh} N \alpha) e^{-N \beta}}{e^{N \delta}-e^{-N \delta}} \\
& B_{n}=-\frac{(4 a N \operatorname{ch} N \beta-2 p \operatorname{sh} N \beta) e^{N \alpha}}{e^{N \delta}-e^{-N \delta}}
\end{aligned}
$$

from which 1.13 (14) follows. The dipole coefficient $e_{x}$ is easily obtained. The alternate expression 1.13 (16) for $e_{x}$ is the result of a familiar transformation.

(b) In the direction perpendicular to the central line (for instance in the $y$-direction) we employ the "tesseral functions"

$$
(2 \operatorname{ch} \eta-2 \cos \theta)^{1 / 2} \sin \theta P_{n}^{\prime}(\cos \theta) \cos \phi e^{ \pm N \eta}
$$

and write

(6) $\quad \psi_{y}=(2 \operatorname{ch} \eta-2 \cos \theta)^{1 / 2} \sum_{n=1}^{\infty}\left(A_{n} e^{N \eta}+B_{n} e^{-N \eta}\right) \sin \theta P_{n}^{\prime}(\cos \theta) \cos \phi$;

the boundary conditions are in this case

$$
\begin{aligned}
& \sum_{n=1}^{\infty}\left(A_{n} e^{N \eta}+B_{n} e^{-N \eta}\right) P_{n}^{\prime}(\cos \theta)=2 a(2 \operatorname{ch} \eta-2 \cos \theta)^{-3 / 2} \\
& \qquad \text { for } \eta=\alpha \text { and } \eta=\beta^{\circ}
\end{aligned}
$$

Differentiating the first identity (3) with respect to $\cos \theta$ and inserting the resulting expansion in (7) we obtain, by comparing the coefficients of $P_{n}^{\prime}(\cos \theta)$,

$$
A_{n}=-\frac{4 a e^{-N \beta} \operatorname{sh} N \alpha}{e^{N \delta}-e^{-N \delta}}, \quad B_{n}=\frac{4 a e^{N \alpha} \operatorname{sh} N \beta}{e^{N \delta}-e^{-N \delta}}
$$

from which 1.13 (17) follows. The dipole coefficient $e_{y}$ is easily obtained.

5.6. Lens and in particular two spheres tangent to each other. The polarization of a lens can be dealt with by using the conal functions $f_{q}(\eta)$ defined in 1.13 (22). The results formulated in 1.13 (e) and 1.14 can be derived without major difficulty. In order to save space we suppress the details of these computations. The main tool is the identity: 


$$
\left(2 \operatorname{ch} \eta+2 \operatorname{ch} \eta^{\prime}\right)^{-1 / 2}=\frac{1}{2 \pi} \int_{-\infty}^{\infty} f_{q}(\eta) \cos q \eta^{\prime} d q
$$

where $\eta>0$ and $\eta^{\prime}$ is arbitrary complex, $\left|\operatorname{Im} \eta^{\prime}\right|<\pi$.

The case of two spheres tangent to each other needs some explanation. We consider both problems of the virtual mass and polarization.

(a) We deal first with the virtual mass. Denoting the radii of the spheres by $r$ and $r^{\prime}$ we apply $1.12(9)$ in the limiting case

$$
\alpha \rightarrow 0, \quad \beta \rightarrow 0, \quad a \rightarrow 0, \quad a / \alpha \rightarrow r, \quad a / \beta \rightarrow r^{\prime} .
$$

From that integral we obtain in the limit

$$
d_{z}=\left(\frac{\pi r r^{\prime}}{r+r^{\prime}}\right)^{3} \frac{\sin \rho}{4 \pi i} \int \frac{d t}{t^{3}} \csc t\{\csc (t+\rho)-\csc (t-\rho)\}, \rho=\frac{\pi r^{\prime}}{r+r^{\prime}} .
$$

The integration is extended along a vertical line $\operatorname{Re}\{t\}=-\epsilon$ where $\epsilon$ is a positive number less than $\rho$ and $\pi-\rho$.

In order to evaluate this integral we observe that the integrand has simple poles at

(4) $t=-n \pi-\rho, t=-(n+1) \pi, t=-(n+1) \pi+\rho, n=0,1,2, \cdots$.

The residues can easily be computed and we obtain

$$
d_{z}=\frac{1}{2}\left(\frac{r r^{\prime}}{r+r^{\prime}}\right)^{3} \sum_{n=0}^{\infty}\left\{\frac{1}{(n+\rho / \pi)^{3}}+\frac{1}{(n+1-\rho / \pi)^{3}}-\frac{2}{(n+1)^{3}}\right\}
$$

which agrees with 1.12 (11).

(b) The formulas for the polarization follow by the same limiting process (2) from the formulas 1.13 (26) and (28). We write $q=a^{-1} \cdot t$ and obtain in the limit:

$$
\begin{gathered}
e_{z}=8 \int_{0}^{\infty} t^{2} \frac{\operatorname{ch} u t+e^{-v t}}{\operatorname{sh} v t} d t-8 \frac{\left(\int_{0}^{\infty} t \frac{\operatorname{sh} u t}{\operatorname{sh} v t} d t\right)^{2}}{\int_{0}^{\infty} \frac{\operatorname{ch} u t-e^{-v t}}{\operatorname{sh} v t} d t}, \\
e_{x}=e_{y}=4 \int_{0}^{\infty} t^{2} \frac{\operatorname{ch} u t-e^{-v t}}{\operatorname{sh} v t} d t
\end{gathered}
$$

where

$$
u=\frac{1}{r}-\frac{1}{r^{\prime}}, \quad v=\frac{1}{r}+\frac{1}{r^{\prime}} .
$$

Replacing the hyperbolic functions by exponentials and expanding, we may express these integrals in terms of the derivatives of $\lambda(s)$. 
5.7. Two-dimensional (cylindrical) problems. Finally we deal with the two-dimensional or cylindrical problem both from the point of view of virtual mass and polarization $\left({ }^{8}\right)$.

Let $C$ be a closed curve in the complex $z$-plane, $z=x+i y=r e^{i \theta}$, and let $\boldsymbol{h}$ be a given vector represented by the complex number $e^{i \alpha}$. The virtual mass $W$ in the direction of $h$ is defined as follows. We form a function $\phi$ (the velocity potential) harmonic outside of $C$, having the form

$$
\phi=\frac{d_{1} \cos \theta+d_{2} \sin \theta}{r}+\frac{d_{1}^{\prime} \cos 2 \theta+d_{2}^{\prime} \sin 2 \theta}{r^{2}}+\cdots
$$

at infinity and satisfying the boundary condition

$$
-\frac{\partial \phi}{\partial \nu}=h \cdot \nu
$$

on $C$.

Then

$$
W=\iint|\operatorname{grad} \phi|^{2} d x d y
$$

integrating outside of $C$.

The polarization $P$ in the direction $h$ is defined as follows. We form the function $\psi$ (electric potential) harmonic outside of $C$, of the form

$$
\psi=\frac{e_{1} \cos \theta+e_{2} \sin \theta}{r}+\frac{e_{1}^{\prime} \cos 2 \theta+e_{2}^{\prime} \sin 2 \theta}{r^{2}}+\cdots
$$

at infinity and satisfying the boundary condition

$$
\psi=\boldsymbol{h} \cdot \boldsymbol{r}+\text { const. }
$$

on $C$.

Then

$$
P=\iint|\operatorname{grad} \psi|^{2} d x d y
$$

integrating outside of $C$.

Both problems can be solved completely by mapping the exterior of the curve $C$ of the $z$-plane conformally onto the exterior of the unit circle $|\xi|>1$, of the $\xi$-plane, with the provision that $z=\infty$ and $\xi=\infty$ correspond to each other. We normalize this mapping in the form:

$$
z=\tau\left(\xi+c_{0}+\frac{c_{1}}{\xi}+\frac{c_{2}}{\xi^{2}}+\cdots\right) \text {. }
$$

The positive number $\tau$ is the "transfinite diameter" of the curve $C$. Now we have, if $z$ and $\zeta$ denote corresponding values,

(8) For the virtual mass of a cylindrical solid, cf. G. P6lya [14]. 


$$
\begin{aligned}
& \phi=\operatorname{Re}\left\{e^{-i \alpha} \tau\left(\xi+\frac{e^{2 i \alpha}}{\xi}+c_{0}\right)-e^{-i \alpha} z\right\} \\
& \psi=\operatorname{Re}\left\{-e^{-i \alpha} \tau\left(\xi-\frac{e^{2 i \alpha}}{\xi}+c_{0}\right)+e^{-i \alpha} z\right\}
\end{aligned}
$$

Indeed,

$$
\boldsymbol{h} \cdot \boldsymbol{r}=x \cos \alpha+y \sin \alpha=\operatorname{Re}\left\{e^{-i \alpha z}\right\}, \quad \boldsymbol{h} \cdot \nu=\frac{\partial}{\partial \nu}(\boldsymbol{h} \cdot \boldsymbol{r})
$$

and $\left(\xi=\rho c^{i \delta}\right)$

$$
\begin{aligned}
\frac{\partial}{\partial \nu} \operatorname{Re}\left\{e^{-i \alpha}\left(\xi+\frac{e^{2 i \alpha}}{\xi}\right)\right\} & =\left|\frac{d \xi}{d z}\right| \frac{\partial}{\partial \rho} \operatorname{Re}\left\{e^{-i \alpha}\left(\xi+\frac{e^{2 i \alpha}}{\xi}\right)\right\}_{\rho=1} \\
& =\left|\frac{d \xi}{d z}\right| \operatorname{Re}\left\{e^{-i \alpha}\left(1-\frac{e^{2 i \alpha}}{\xi^{2}}\right) \xi\right\}_{\rho=1}=0 .
\end{aligned}
$$

The verification of the boundary condition for $\psi$ is even simpler.

As to the virtual mass and polarization,

$$
\begin{aligned}
W & =\int_{1}^{\infty} \int_{-\pi}^{\pi}\left|e^{-i \alpha} \tau\left(1-\frac{e^{2 i \alpha}}{\xi^{2}}\right)-e^{-i \alpha} \frac{d z}{d \xi}\right|^{2} \rho d \rho d \delta, \\
P & =\int_{1}^{\infty} \int_{-\pi}^{\pi}\left|-e^{-i \alpha} \tau\left(1+\frac{e^{2 i \alpha}}{\xi^{2}}\right)+e^{-i \alpha} \frac{d z}{d \xi}\right|^{2} \rho d \rho d \delta
\end{aligned}
$$

and an easy calculation shows (cf. Pólya [14, p. 220]) that

$$
\begin{gathered}
W=-A+2 \pi \tau^{2}\left\{1-\operatorname{Re}\left(c_{1} e^{-2 i \alpha}\right)\right\}, \\
P=-A+2 \pi \tau^{2}\left\{1+\operatorname{Re}\left(c_{1} e^{-2 i \alpha}\right)\right\},
\end{gathered}
$$

where

$$
A=\pi \tau^{2}\left(1-\left|c_{1}\right|^{2}-2\left|c_{2}\right|^{2}-3\left|c_{3}\right|^{2}-\cdots\right)
$$

is the area of the curve $C$, that is, of the cross section of the cylinder. The minimum property of the circular cylinder with respect to the virtual mass pointed out by Pólya (loc. cit., p. 221) holds good without change with respect to the polarization.

The analogue of the formulas 1.2 (2) holds again replacing $4 \pi$ by $2 \pi$. Indeed, the leading term of (1) is, in view of (8),

$$
\operatorname{Re}\left\{e^{-i \alpha} \tau \frac{e^{2 i \alpha}-c_{1}}{\xi}\right\} \quad \text { or } \quad \operatorname{Re}\left\{e^{-i \alpha} \tau^{2} \frac{e^{2 i \alpha}-c_{1}}{z}\right\}=\frac{d_{1} \cos \theta+d_{2} \sin \theta}{r}
$$

so that the strength-component in the $h$-direction will be: 
(16) $D=d_{1} \cos \alpha+d_{2} \sin \alpha=\operatorname{Re}\left\{e^{-2 i \alpha} \tau^{2}\left(e^{2 i \alpha}-c_{1}\right)\right\}=\tau^{2}\left[1-\operatorname{Re}\left(c_{1} e^{-2 i \alpha}\right)\right]$. Hence (13) can be written as follows:

$$
W=2 \pi D-A .
$$

A similar representation is possible for the polarization.

\section{BiBLIOGRAPHY}

1. A. B. Basset, On the potential of an electrified spherical bowl, and on the velocity potential due to the motion of an infinite liquid about such a bowl, Proc. London Math. Soc. vol. 16 (1885) pp. 286-306.

2. - On the motion of two spheres in a liquid, and allied problems, Proc. London Math. Soc. vol. 18 (1887) pp. 369-377.

3. S. Bergman and M. Schiffer, A representation of Green's and Neumann's functions in the theory of partial differential equations of second order, Duke Math. J. vol. 14 (1947) pp. 609638.

4. J. B. Díaz and A. Weinstein, The torsional rigidity and variational methods, Amer. J. Math. vol. 70 (1948) pp. 107-116.

5. J. Hadamard, Leģons sur le calcul des variations, Paris, 1910. 204,370 .

6. R. A. Herman, On the motion of two spheres in fluid, Quart. J. Math. vol. 22 (1887) pp.

7. W. M. Hicks, On the motion of two spheres in a fluid, Philos. Trans. Roy. Soc. London Ser. A. vol. 171 (1880) pp. 455-492.

8. —- On toroidal functions, Philos. Trans. Roy. Soc. London Ser. A. vol. 172 (1881) pp. $609-652$.

9. - Report on recent papers in hydrodynamics, British Association for the Advancement of Science vol. 1 (1882) pp. 57-58; vol. 2 (1883) pp. 39-70.

10. H. Lamb, Hydrodynamics, 6th ed., 1932; Dover, 1945.

11. L. Lichtenstein, Potentialtheorie, Konforme Abbildung, Enzyclopädie der mathematischen Wissenschaften II C 3.

12. C. Neumann, Hydrodynamische Untersuchungen, Leipzig, 1883.

13. H. Poincare, Figures d'équilibre d'une masse fluide, Paris, 1902.

14. G. Pólya, A minimum problem about the motion of a solid through a fluid, Proc. Nat. Acad. Sci. U.S.A. vol. 33 (1947) pp. 218-221.

15. G. Pólya and G. Szegö, Inequalities for the capacity of a condenser, Amer. J. Math. vol. 67 (1945) pp. 1-32.

16. - Approximations and bounds for the electrostatic capacity and for similar physical quantities, To appear in Memoirs of the American Mathematical Society.

17. M. Shiffman and D. C. Spencer, The flow of an ideal incompressible fluid about a lens, Quarterly of Applied Mathematics vol. 5 (1947) pp. 270-288. 350.

18. G. Szegö, On the capacity of a condenser, Bull. Amer. Math. Soc. vol. 51 (1945) pp. 325209-223.

19. - On the virtual mass of nearly spherical solids, Duke Math. J. vol. 16 (1949) pp.

20. G. I. Taylor, The energy of a body moving in an infinite fluid, with an application to airships, Proc. Roy. Soc. London Ser. A. vol. 120 (1928) pp. 13-21.

HEBREW UNIVERSITY, JERUSALEM.

StANFORD UNIVERSITY, Stanford University, Calif. 